\title{
Comportement Asymptotique de Solutions de Certains Problèmes Mixtes pour des Systèmes Hyperboliques Symétriques à Coefficients Constants
}

\author{
Par \\ Mutsuhidé MatsumuRA*
}

\section{\$1. Introduction}

Le comportement asymptotique pour $t \rightarrow \infty$ de solutions du problème mixte extérieur pour l'équation des ondes dans l'espace à trois dimensions a été étudié par quelques auteurs, par exemple T. Carleman [2], P.D. Lax et R.S. Phillips [5], P.D. Lax, C.S. Morawetz et R.S. Phillips [6], C.S. Morawetz [11], S. Mizohata [9] et d'autres. Dans [5], P.D. Lax et R.S. Phillips ont aussi établi la décroissance locale de l'énergie pour des solutions de systèmes hyperboliques symétriques dans un domaine extérieur de l'espace euclidien à dimension impaire.

Ces travaux s'occupent du cas de l'obstacle borné. Il nous semble aussi intéressant d'étudier des problèmes de ce genre dans le demiespace. Dans le travail antérieur [7], nous avons établi la décroissance pour $t \rightarrow \infty$ des solutions de certains problèmes mixtes dans le demi-espace $\boldsymbol{R}_{+}^{n}$ pour des systèmes hyperboliques symétriques du premier ordre à coefficients constants. Mais nos hypothèses sur les systèmes et sur les conditions aux limites, faites dans [7] sont assez restrictives. En effet, nous avons traité dans [7] seulement les systèmes isotropes (voir Remarque 2 dans le $\S 1$ ) et les conditions aux limites vérifiant la condition complémentaire stricte (ou de

Received November 8, 1969.

Communicated by S. Matsuura.

* Department of Applied Mathematics and Physics, Faculty of Engineering, Kyoto University. 
Lopatinski uniforme). ${ }^{\dagger}$ Dans cet article, en construisant le noyau compensateur d'un point de vue différent de [7], nous établirons la décroissance de solutions pour des systèmes et des conditions aux limites plus généraux. Cet article est complet par lui-même. Par suite le lecteur pourra lire cet article indépendamment de $[7] .^{\dagger \dagger}$

A la fin, notons que sous des conditions plus restrictives que celles de P.D. Lax et R.S. Phillips, K. Mochizuki [11] a obtenu tout récemment quelques résultats de ce genre pour des problèmes mixtes de systèmes hyperboliques symétriques à coefficients variables dans un domaine extérieur d'un obstacle borné de l'espace à dimension paire.

\section{Quelques rappels sur le problème mixte.}

On désigne par $\boldsymbol{R}^{n}$ l'espace euclidien à $n$ dimensions, par $x=\left(x_{1}, \cdots, x_{n}\right)$ un point de $\boldsymbol{R}^{n}$ et par $|x|$ sa distance à l'origine. En posant $x^{\prime}=\left(x_{1}, \cdots, x_{n-1}\right)$, écrivons $x=\left(x^{\prime}, x_{n}\right)$. Soit $\boldsymbol{R}_{+}^{n}$ le demi-espace ouvert $\left\{x=\left(x^{\prime}, x_{n}\right) \in \boldsymbol{R}^{n} ; x_{n}>0\right\}$ et soit $\overline{\boldsymbol{R}}_{+}^{n}$ la fermeture de $\boldsymbol{R}_{+}^{n} . t$ désigne la variable de temps.

Soit $L$ un opérateur hyperbolique symétrique du premier ordre à coefficients constants :

$$
L=I \frac{\partial}{\partial t}-\sum_{j=1}^{n} A_{j} \frac{\partial}{\partial x_{j}}
$$

où $I$ est la matrice unité d'ordre $N$ et les $A_{j}, j=1, \cdots, n$ sont des matrices hermitiennes d'ordre $N$ dont les éléments sont constants. Nous considérons le problème mixte, dans le demi-espace $\boldsymbol{R}_{+}^{n}$, de la forme :

$$
\begin{array}{cl}
L[u(t, x)]=0 & \text { pour } t>0, x \in \boldsymbol{R}_{+}^{n}, \\
u(0, x)=g(x) & \text { pour } \quad x \in \boldsymbol{R}_{+}^{n}, \\
B u(t, x)_{x_{n}=0}=0 & \text { pour } t>0,
\end{array}
$$

où $u(t, x)$ et $g(x)$ sont des matrices-colonnes de fonctions à valeurs

† Cela correspond à la condition complémentaire de S. Agmon [1] dans les équations hyperboliques d'ordre supérieur (voir la condition B.2)* dans le $\$ 7$ : Appendice). †† Les résultats de cet article ont été résumés dans [8]. 
complexes et $B$ une matrice à $l$ lignes et $N$ colonnes de rang $l$, d'éléments constants.

Notre but est de montrer la décroissance pour $t \rightarrow \infty$ de la solution $u(t, x)$ du problème mixte (1.2)-(1.4) avec $g \in C_{0}^{\infty}\left(\boldsymbol{R}_{+}^{n}\right)$ sous les hypothèses sur $L$ et $B$ que nous énoncerons précisément plus tard. Pour cela, rappelons d'abord quelques notions et la terminologie concernant le problème mixte (voir aussi S. Agmon [1], R. Hersh [3], P.D. Lax et R.S. Phillips [5]).

Soit $\boldsymbol{C}^{N}$ l'espace complexe de dimension complexe $N$. Désignons par la même lettre $B$ l'opérateur linéaire de $C^{N}$ dans $C^{l}$ dont la matrice de représentation par rapport aux bases canoniques de $C^{N}$ et de $C^{l}$ est la matrice $B$. On l'appelle opérateur-frontière. On appelle aussi le noyau de cet opérateur linéaire espace-frontière et désigne par $\mathscr{B}=\operatorname{ker} B$.

Définition 1.1. On dit que la condition $B$ aux limites (ou l'opérateur-frontière $B$ ou encore l'espace-frontière) est conservative si la forme quadratique $A_{n} \zeta \cdot \bar{\zeta}$ (associée à $A_{n}$ ) s'annule sur $\mathscr{B}$ :

$$
A_{n} \zeta \cdot \bar{\zeta}=0 \quad \text { pour tout } \zeta \in \mathscr{B} \text {. }
$$

Pour $\zeta, \zeta^{\prime} \in C^{N}, \zeta \cdot \bar{\zeta}^{\prime}$ désigne le produit scalaire $\zeta_{1} \bar{\zeta}_{1}^{\prime}+\cdots+\zeta_{N} \bar{\zeta}_{N}{ }^{\prime}$.

On peut aisément constater que si $B$ est conservatif, toute solution $u(t, \cdot) \in L^{2}\left(\boldsymbol{R}_{+}^{n}\right)$ du problème mixte (1.2)-(1.4) satisfait à la loi qui exprime la conservation de l'énergie de la forme intégrale:

$$
\|u(t, \cdot)\|_{L^{2}\left(\boldsymbol{R}_{+}^{n}\right)}=\|u(0, \cdot)\|_{L^{2}\left(\boldsymbol{R}_{+}^{n}\right)} .
$$

Cette égalité implique évidemment l'unicité de la solution et sa dépendance continue des conditions initiales. Mais pour obtenir un théorème général d'existence on a en outre besoin de la notion suivante qui exclut l'excès de relations aux limites.

Définition 1.2. On dit que l'espace-frontière $\mathscr{B}$ est maximal par rapport à la propriété (1.5) (ou plus simplement, maximalement conservatif) s'il est impossible d'élargir $\mathcal{B}$ à un sous-espace de $\boldsymbol{C}^{N}$ strictement plus large en gardant la propriété (1.5).

On peut alors appeler la condition $B$ (ou l'opérateur-frontière) minimal par rapport à la propriété (1.5) ou minimalement con- 
servative dans le sens qu'en gardant la propriété (1.5) on ne peut omettre aucune ligne de la matrice $B$ qui stipule les $l$ relations linéaires homogènes parmi les composantes de $u(t, x)$ à le frontière $x_{n}=0$.

Nous citons ici le lemme suivant dû à P.D. Lax et R.S. Phillips [5] qui élucide la structure des sous-espaces jouissant de la propriéte (1.5) d'une façon maximale pour une matrice $A_{n}$ hermitienne, non singulière de signature zéro.

Lemme 1.1. ([5] p. 199) Soit $Q$ une matrice hermitienne d'ordre $2 m$, non singulière et de signature zéro. On désigne par $\mathscr{Q}$ (resp. $\Re)$ le sous-espace de dimension $m$ engendré par les vecteurs propres correspondant aux valeurs propres positives (resp. négatives) de $Q$. Soit $\left\{\boldsymbol{e}_{1}^{+}, \cdots, \boldsymbol{e}_{m}^{+}\right\}$(resp. $\left\{\boldsymbol{e}_{1}^{-}, \cdots, \boldsymbol{e}_{m}^{-}\right\}$une base orthonormale quelconque dans $\mathscr{Q}$ par rapport $\dot{a} Q$ (resp. dans $\mathcal{N}$ par rapport $\dot{a}-Q$ ). Désignons par $\mathcal{E}$ le sous-espace de $\boldsymbol{C}^{2 m}$ engendré par les vecteurs $\boldsymbol{e}_{1}^{+}+\boldsymbol{e}_{1}^{-}, \boldsymbol{e}_{2}^{+}+\boldsymbol{e}_{2}^{-}, \cdots, \boldsymbol{e}_{m}^{+}+\boldsymbol{e}_{m}^{-}$. On a alors

$$
Q \zeta \cdot \bar{\zeta}=0 \text { pour tout } \zeta \in \mathcal{E},
$$

et $\mathcal{E}$ est maximal par rapport à cette propriété. Inversement tout sous-espace de $\boldsymbol{C}^{2 m}$ ayant la propriété (1.5)' d'une façon maximale, peut être construit par un tel procédé.

Posons pour simplifier la notation:

$$
\begin{aligned}
& A=\frac{1}{i} \sum_{j=1}^{n} A_{j} \frac{\partial}{\partial x_{j}}, \\
& A(\eta)=\sum_{j=1}^{n} \eta_{j} A_{j} .
\end{aligned}
$$

$\eta$ désigne ici un point de l'espace $\boldsymbol{g}^{n}$ dual réel de $\boldsymbol{R}^{n}$ par rapport au produit scalaire $\langle x, \eta\rangle=\sum_{j=1}^{n} x_{j} \eta_{j}=x \cdot \eta$.

Nous posons aussi

$$
A^{\prime}\left(\eta^{\prime}\right)=\sum_{j=1}^{n-1} \eta_{j} A_{j}
$$

en ecrivant $\eta^{\prime}=\left(\eta_{1}, \cdots, \eta_{n-1}\right)$. Dans ce qui suit, nous écrirons très souvent $\eta_{n}=\tau, \eta^{\prime}=\left(\eta_{1}, \cdots, \eta_{n-1}\right)=\xi=\left(\xi_{1}, \cdots, \xi_{n-1}\right)$ et $\eta=(\xi, \tau)$. 
Supposons désormais que l'hyperplan $x_{n}=0$ ne soit pas caractéristique pour $L$ (ou $A$ ), à savoir que dét $A_{n} \neq 0$, et considérons la matrice suivante dépendant des paramètres $\xi \in \boldsymbol{\Xi}^{n-1}$ et $\lambda \in \boldsymbol{C}$.

$$
M(\xi ; \lambda)=A_{n}^{-1}\left(\lambda I-A^{\prime}(\xi)\right) .
$$

Alors il découle de l'hyperbolicité de $L=I \frac{\partial}{\partial t}-i A$ que pour tout vecteur réel $\xi \in \Xi^{n-1}$ et pour tout $\lambda$ non réel, aucune des racines caractéristiques de $M(\xi ; \lambda)$ n'est réelle.

Pour tout $\xi$ réel et $\neq 0$, la matrice $M(\xi ; 0)$ a la même propriété si l'opérateur $A$ est de type elliptique dans le sens que $A(\eta)$ est non singulier pour tout $\eta \neq 0$ dans $\Xi^{n}$.

Désignons maintenant par $E^{+}(\xi ; \lambda)$ (resp. $E^{-}(\xi ; \lambda)$ ) le sousespace de $\boldsymbol{C}^{N}$ engendré par les vecteurs propres ordinaires et généralisés correspondant aux valeurs propres $(=\text { racines caractéristiques) })^{1)}$ à partie imaginaire positive (resp. négative) de $M(\xi ; \lambda)$. On appelle $E^{+}(\xi ; \lambda)$ (resp. $E^{-}(\xi ; \lambda)$ ) espace propre positif (resp. négatif) de $M(\xi ; \lambda)$.

Pour tout $\xi$ réel $\xi^{n-1}$ et $\lambda$ non réel, on a

$$
E^{\vdash}(\xi ; \lambda) \oplus E^{-}(\xi ; \lambda)=\boldsymbol{C}^{N} .
$$

Supposons maintenant que l'opérateur $A$ soit de type elliptique. Alors on a, pour $\xi \in \Xi^{n-1}$ et $\lambda$ non réel et pour $\xi \neq 0$ et $\lambda=0$, (1.10) et de plus

$$
\operatorname{dim} E^{+}(\xi ; \lambda)=\operatorname{dim} E^{-}(\xi ; \lambda)=\frac{N}{2}, \text { si } n>2 .
$$

Lorsque $n=2$, nous supposons (1.11), à savoir que $A$ soit proprement elliptique.

Définition 1.3. On dit qu'une condition $B$ aux limites (ou l'opérateur-frontière $B$, ou encore l'espace-frontière) est coercive pour l'opérateur elliptique $A$ s'il existe une constante $C>0$ telle qu'on ait

$$
\sum_{j=1}^{n}\left\|\frac{\partial v}{\partial x_{j}}\right\| \leqslant C(\|A v\|+\| v ! \mid)
$$

1) Nous appelons souvent les racines caractéristiques valeurs propres lorsque nous utilisons ces racines avec vecteurs propres correspondants. 
pour toute fonction $v \in C_{0}^{\infty}\left(\boldsymbol{R}_{+}^{n}\right)$ vérifiant la condition aux limites $B v(x)_{x_{n}=0}=0 . \quad\|\cdot\|$ désigne ici la norme naturelle de $L^{2}\left(\boldsymbol{R}_{+}^{n}\right)$.

Le lemme suivant doit aussi à P.D. Lax et R.S. Phillips [5] (p. 202).

Lemme 1.2. Pour qu'une condition $B$ aux limites soit coercive pour l'opérateur elliptique symétrique $A=-i \sum_{j=1}^{n} A_{j} \frac{\partial}{\partial x_{j}}$, il faut et il suffit que les conditions suivantes soient vérifiées.

i) $\quad l=\frac{N}{2} \quad$ et

ii) $\mathscr{B} \cap E^{+}(\xi ; 0)=0$ pour tout $\xi \neq 0$ dans $\Xi^{n-1}$.

$l$ est ici le nombre des lignes de la matrice $B$.

\section{Hypothèses et théorème principal.}

Nous imposons ici un certain nombre de conditions sur $L$ et sur $B$.

(I) "hypothèses sur l'opérateur L"

L. 1) $L$ est strictement hyperbolique, cela signifie que pour tout vecteur réel $\eta=\left(\eta_{1}, \cdots, \eta_{n}\right) \neq 0$, les racines caractéristiques de $A(\eta)$ sont toutes réelles et distinctes :

$$
\begin{gathered}
\operatorname{dét}(\lambda I-A(\eta))=\left(\lambda-\lambda_{1}(\eta)\right) \cdots\left(\lambda-\lambda_{N}(\eta)\right), \\
\lambda_{1}(\eta)>\cdots>\lambda_{N}(\eta) \text { pour } \eta \neq 0 .
\end{gathered}
$$

L. 2) $A$ est elliptique, en d'autres termes, $L$ n'a pas de vitesses de propagation nulles,

L. 3) Pour tout $\xi$ réel et $\lambda=k$ réel $\neq 0$, les racines caractéristiques réelles de $M(\xi ; k)$ sont ou simples ou doubles. ${ }^{2)}$ De plus $M(\xi ; k)$ n'a qu'un couple de racines doubles réelles tout au plus pour le même $(\xi, k)$.

(II) "hypothèses sur la condition B aux limites"

B. 1) $B$ est minimalement conservatif.

2) C'est la condition d'Agmon [1]. 
B. 2) Pour tout vecteur $\xi$ réel $\in \Xi^{n-1}$ et pour $\lambda=k$ réel, $\xi$ et $k$ non tous deux nuls, on a

$$
\mathscr{B} \cap E^{+}(\xi ; k)=\{0\} .
$$

Remarque 1. De l'hypothèse L. 1), il découle que $\lambda_{j}(\xi) j=1, \cdots, n$ sont des fonctions analytiques réelles dans $\Xi^{n}-\{0\}$ et positivement homogènes de degré un.

Remarque 2. Voici une classe d'opérateurs typiques vérifiant les conditions L. 1)-L. 3) :

$$
\begin{gathered}
\operatorname{dét}(\lambda I-A(\eta))=\left(\lambda^{2}-a_{1}^{2}|\eta|^{2}\right) \cdots\left(\lambda^{2}-a_{m}^{2}|\eta|^{2}\right), \\
a_{1}>\cdots>a_{m}>0,
\end{gathered}
$$

c'est-à-dire le cas isotrope à vitesses de propagation non nulles.

Remarque 3. La condition B. 2) contient la coercivité de $B$ pour l'opérateur elliptique symétrique $A$ (voir Lemme 2.1).

Remarque 4. Notons que le sous-espace $E^{+}(\xi ; k)$ ne coïncide pas avec $E^{+}(\xi ; k \pm i 0)$ pour un choix convenable de $(\xi, k)$.

Remarque 5. De la symétrie des $A_{j}, j=1, \cdots, n$ et la propriété d'être conservative de $B$, on peut déduire

$$
\mathscr{B} \cap E^{+}(\xi ; \lambda)=\{0\}
$$

pour tout $\xi \in \Xi^{n-1}$ et pour tout $\lambda$ non réel (voir Lemme 5. 3).

Nous allons maintenant énoncer notre théorème principal.

Théorème. Supposons que l'opérateur $L$ et la condition $B$ aux limites satisfassent à toutes les conditions énumérées ci-dessus. Soit $g \in C_{0}^{\infty}\left(\boldsymbol{R}_{+}^{n}\right)$. Alors la solution $u(t, x)$ du probleme mixte (1.2)-(1.4) converge vers zéro uniformément sur tout compact de $\boldsymbol{R}_{+}^{n}$ lorsque $t$ tend vers l'infini. De plus, toutes les dérivées de $u(t, x)$ par rapport $\dot{a}(t, x)$ ont la même propriété.

Nous allons ici indiquer en bref le principe de la démonstration du théorème. Nous traiterons ce problème dans le cadre des espaces $L^{2}$. A cause de la symétrie des $A_{j}$ et de l'hypothèse B. 1), l'opérateur $A$ devient un opérateur autoadjoint $\boldsymbol{A}$ dans $L^{2}\left(\boldsymbol{R}_{+}^{n}\right)$ en prenant pour 
domaine

$$
D(\boldsymbol{A})=\left\{v(x) ; v \in H^{1}\left(\boldsymbol{R}_{+}^{n}\right), B v_{x_{n}=0}=0\right\},
$$

$H^{1}\left(\boldsymbol{R}_{+}^{n}\right)$ désignant ici l'espace des fonctions $v \in L^{2}\left(\boldsymbol{R}_{+}^{n}\right)$ dont toutes les dérivées distributions d'ordre un, sont dans $L^{2}\left(\boldsymbol{R}_{+}^{n}\right)$. Alors en utilisant cet opérateur fermé $\boldsymbol{A}$, le problème mixte (1.2)-(1.4) peut s'écrire sous la forme de l'équation d'évolution

$$
\begin{aligned}
& \frac{d}{d t} u(t)=i A u(t), \quad t>0 \\
& u(0)=g \in C_{0}^{\infty}\left(\boldsymbol{R}_{+}^{n}\right) \subset D(\boldsymbol{A}) .
\end{aligned}
$$

Par conséquent la solution est donnée par

$$
u(t)=e^{i t_{A}} g=\int_{-\infty}^{+\infty} e^{i k t} d \mathbb{E}_{k} g
$$

où $\left\{\boldsymbol{E}_{\boldsymbol{k}}\right\}_{-\infty<k<+\infty}$ est la famille spectrale de l'opérateur autoadjoint $\boldsymbol{A}$ dans l'espace $L^{2}\left(\boldsymbol{R}_{+}^{n}\right)$. La démonstration du théorème revient alors en essence à prouver que $\boldsymbol{E}_{k} g$ est absolument continue en $k$ à l'exception de l'origine $k=0$. Pour cela nous construirons d'abord le noyau de Green $G(x, y ; \lambda)$ du problème stationnaire associé au problème mixte (1.2)-(1.4) pour l'opérateur hyperbolique $L$, plus précisément du problème aux limites dans $\boldsymbol{R}_{+}^{n}$ pour l'opérateur elliptique $A-\lambda I(\lambda$ non réel $)$ :

$$
\begin{aligned}
&(A-\lambda I) v(x)=g(x), \quad x \in \boldsymbol{R}_{+}^{n}, \\
& B v(x)_{x_{n}=0}=0 .
\end{aligned}
$$

Ensuite nous montrons que pour tout indice multiple $\nu$, les limites

$$
G_{k \pm i 0}^{(\nu)} \cdot g(x)=\lim _{\varepsilon \downarrow 0}\left(\frac{\partial}{\partial x}\right)^{\nu} \int_{\mathbb{R}_{+}^{n}} G(x, y ; k \pm i \varepsilon) g(y) d y
$$

existent et qu'elles sont des fonctions continues de $(x, k)$ dans $\boldsymbol{R}_{+}^{n} \times(\boldsymbol{R}-\{0\})$. Alors nous pouvons établir, en utilisant la formule d'inversion de Stieltjes, la relation suivante.

$$
\frac{d}{d k} \mathbb{E}_{k} g(x)=\frac{1}{2 \pi i} \int_{\mathbb{R}_{+}^{n}}\{G(x, y ; k+i 0)-G(x, y ; k-i 0)\} g(y) d y .
$$


On observe que pour tout $x$ fixé le second membre de cette formule est une fonction continue de $k$ dans $\boldsymbol{R}-\{0\}$. D'autre part on peut déduire de la coercivité de $B$ que $k=0$ n'est pas une valeur propre de $\boldsymbol{A}$. Le théorème voulu se démontre alors en appliquant le théorème de Riemann-Lebesgue à l'expression (1.18).

Donc, le point essentiel de notre travail consiste à construire le noyau de Green $G(x, y ; \lambda)$ du problème (1.19)-(1.20) et à montrer (1.21).

On cherche le noyau $G(x, y ; \lambda)$ sous la forme:

$$
G(x, y ; \lambda)=E(x-y ; \lambda)-E_{c}(x, y ; \lambda), x, y \in \boldsymbol{R}_{+}^{n} .
$$

$E(x ; \lambda)$ est ici une solution élémentaire de l'opérateur $A-\lambda I$ dans $\boldsymbol{R}^{n}$ qui est définie par

$$
E(x ; \lambda)=(2 \pi)^{-(n / 2)} \mathscr{F}^{-1}\left[(A(\xi)-\lambda I)^{-1}\right],
$$

$\mathscr{F}^{-1}$ désignant la transformation de Fourier inverse. D'autre part $E_{c}(x, y ; \lambda)$ est ce que l'on appelle noyau compensateur et il s'obtient comme solution du problème aux limites:

$$
\begin{aligned}
& (A-\lambda I) E_{c}(x, y ; \lambda)=0, \quad x, y \in \boldsymbol{R}_{+}^{n} \\
& \left.B E_{c}(x, y ; \lambda)\right|_{x_{n}=0}=B E(x-y ; \lambda) \underset{x_{n}=0}{\mid} .
\end{aligned}
$$

En effectuant formellement la transformation de Fourier partielle par rapport aux variables $x^{\prime}=\left(x_{1}, \cdots, x_{n-1}\right)$, on a le système d'équations différentielles ordinaires de la seule variable $x_{n}$, dépendant des paramètres $\xi$ et $\lambda$ :

$$
\left(\frac{1}{i} \frac{d}{d x_{n}}-M(\xi ; \lambda)\right) \widetilde{E}_{c}\left(\xi, x_{n}, y ; \lambda\right)=0, x_{n}>0,
$$

et la condition

$$
B \widetilde{E}_{c}(\xi, 0, y ; \lambda)=\mathscr{I}_{x^{\prime}}\left[B E(x-y ; \lambda)_{x_{n}=0}\right] .
$$

$\mathscr{F}_{x^{\prime}}$ désigne ici la transformation de Fourier partielle par rapport à $x^{\prime}$ et $\widetilde{E}_{c}=\mathscr{F}_{x^{\prime}}\left[E_{c}\right]$. Ainsi le problème de la construction de $G(x, y ; \lambda)$ se réduit à trouver la solution de (1.27)-(1.28) dans $L^{2}\left(\left\{x_{n} ; x_{n}>0\right\}\right)$.

Le plan du reste de cet article est le suivant. Le $\$ 2$ est con- 
sacré à quelques considérations sur les racines caractéristiques de la matrice $M(\xi ; \lambda)$ et à la construction de bases dans les espaces $E^{ \pm}(\xi ; \lambda)$. Dans le $\S 3$, nous donnons d'abord un certain nombre de lemmes. Puis nous démontrons un théorème sur l'ordre des zéros du déterminant de Lopatinski $\Delta(\xi ; k \pm i 0)$. Dans le $\S 4$, nous définissons une solution élémentaire de l'opérateur $A-\lambda I$ pour $\lambda$ non réel dans l'espace libre $\boldsymbol{R}^{n}$ et montrons l'existence de $E(x ; k \pm i 0)$ et leur continuité par rapport à $(x, k)$ dans $\{(x, k) ; x \neq 0, k \neq 0\}$. Ensuite nous donnons une expression de $\mathscr{F}_{x}\left(B E(x-y ; \lambda)_{x_{n}=0}\right]$. Dans le $\S 5$, nous donnons une expression de $\mathscr{F}_{x}{ }_{x}\left[E_{c}(x, y ; \lambda)\right]$ et en utilisant cette expression nous montrons l'existence de $E_{c}(x, y ; k \pm i 0)$ et leur continuité par rapport à $(x, y, k)$ dans $\left\{(x, y, k), x, y \in \boldsymbol{R}_{+}^{n}\right.$ et $k \neq 0\}$. Le $\S 6$ est consacré à la démonstration complète de notre théorème principal. Enfin, nous donnons des exemples dans l'appendice.

En terminant cette introduction, je voudrais exprimer ma vive gratitude aux professeurs S. Mizohata et M. Yamaguti pour leurs conseils bienveillants.

\section{§2. Considérations Préliminaires}

Considérations sur les racines caractéristiques de la matrice $M(\xi ; \lambda)$.

Soient $k^{0}$ un nombre réel $\neq 0$ et $\xi^{0}$ un point de $g^{n-1}$. Supposons maintenant que la matrice $M\left(\xi^{0} ; k^{0}\right)$ admette une racine caractéristique réelle $\sigma^{0}$. Alors d'après l'hyperbolicité stricte de $L$ et la relation :

$$
\operatorname{dét}(\lambda I-A(\xi, \tau))=\operatorname{dét}\left(-A_{n}\right) \cdot \operatorname{dét}(\tau I-M(\xi ; \lambda)) \text {, }
$$

il existe uniquement un numéro $r$ tel que $1 \leqslant r \leqslant N$ et

$$
k^{0}=\lambda_{r}\left(\xi^{0}, \sigma^{0}\right) \text {. }
$$

On sait que $\lambda(\xi, \sigma)$ est une fonction analytique à valeurs réelles des variables réelles $\xi=\left(\xi_{1}, \cdots, \xi_{n-1}\right)$ et $\sigma$ dans $\{(\xi, \sigma) ;|\xi|+|\sigma| \neq 0\}$ (voir Remarque 1 dans le $\S 1$ ). Nous considérons la fonction $\lambda=\lambda_{r}(\xi, \sigma)$ au voisinage du point $\left(\xi^{0}, \sigma^{0}\right)$ et prolongeons la dernière variable $\sigma$ en variable complexe $z$. Plus tard, nots remplacerons $z$ par $\tau$. Désormais la lettre $\sigma$ désignera toujours une variable réelle ou $\operatorname{Re} z$. Nous utiliserons souvent par la suite la lettre $\lambda$ comme une 
variable complexe indépendante. Lorsque $\lambda$ décrit seulement la droite, à savoir que $\lambda$ jouera le rôle d'une variable réelle, nous emploierons $k$ au lieu de $\lambda$.

A propos, la racine caractéristique réelle $\sigma^{0}$ est ou simple ou double par hypothèse. Considérons d'abord le cas où $\sigma^{0}$ est simple. Cela équivaut à

$$
\frac{\partial \lambda_{r}}{\partial z}\left(\xi^{0}, \sigma^{0}\right) \neq 0
$$

En effet supposons que $\frac{\partial \lambda_{r}}{\partial z}\left(\xi^{0}, \sigma^{0}\right)=0$. Soit $l$ le premier numéro tel que $\frac{\partial^{l} \lambda_{r}}{\partial z^{l}}\left(\xi^{0}, \sigma^{0}\right) \neq 0$. Alors $z=\sigma^{0}$ est un $k^{0}$-point d'ordre $l$ de la fonction $\lambda=\lambda_{r}\left(\xi^{0}, z\right)$ en $z$. Donc il existe, d'après un théorème bien connu dans la théorie des fonctions, la fonction $z=z\left(\xi^{0} ; \lambda\right)$ définie dans un voisinage du point $\lambda=k^{0}$, ayant un point critique algébrique $k^{0}$ d'ordre $l-1 . \quad z\left(\xi^{0} ; \lambda\right)$ est alors une racine caractéristique de $M\left(\xi^{0} ; \lambda\right)$ et on aura une racine multiple réelle $\sigma^{0}=z\left(\xi^{0} ; k^{0}\right)$ d'ordre $l \geqslant 2$, contrairement à l'hypothèse que $\sigma^{0}$ est simple. On a donc (2.3). De (2.3) il existe, en vertu du théorème d'existence des fonctions implicites, une fonction analytique $z=z(\xi ; \lambda)$ définie au voisinage du point $(\xi, z)=\left(\xi^{0}, \sigma^{0}\right)$, ayant les propriétés :

i) $z(\xi ; \lambda)$ vérifie

$$
\sigma^{0}=z\left(\xi^{0} ; k^{0}\right) \quad \text { et } \quad \lambda \equiv \lambda_{r}(\xi, z(\xi ; \lambda)) .
$$

ii) $z(\xi ; k)$ est une fonction à valeurs réelles.

Alors $z=\boldsymbol{z}(\xi ; \lambda)$ est racine caractéristique simple de $M(\xi ; \lambda)$ pour tout $(\xi, \lambda)$ dans un petit voisinage de $\left(\xi^{0}, k^{0}\right)$ et $\operatorname{Im} z(\xi ; \lambda) \neq 0$ pour $\lambda$ non réel.

Nous allons maintenant montrer que l'on a

$$
\lim _{\operatorname{Im} \lambda \rightarrow 0} \frac{\operatorname{Im} \lambda}{\operatorname{Im} z(\xi ; \lambda)}=\frac{\partial \lambda_{r}}{\partial z}(\xi, z(\xi ; k)) \neq 0
$$

pour $(\xi, k)$ voisin de $\left(\xi^{0}, k^{0}\right)$, où $k=\operatorname{Re} \lambda$.

Pour cela développons la fonction $\lambda=\lambda_{r}(\xi, z)$ en série de Taylor rapport à $z$ au voisinage de $z=\sigma$ voisin de $\sigma^{0}$.

$$
\lambda_{r}(\xi, z)=\sum_{l=0}^{\infty} \frac{1}{l !} \frac{\partial^{l} \lambda_{r}}{\partial z^{l}}(\xi, \sigma)(z-\sigma)^{l} .
$$


En remplaçant ici $z$ et $\sigma$ par $z=z(\xi ; \lambda)$ et $\sigma=z(\xi ; k)(k=\operatorname{Re} \lambda)$ respectivement, on a l'identité

$$
\lambda-k=\sum_{l=1}^{\infty} \frac{1}{l !} \frac{\partial^{l} \lambda}{\partial z^{l}}(\xi, z(\xi ; k))(z(\xi ; \lambda)-z(\xi ; k))^{l} .
$$

En prenant les parties imaginaires, il vient

$\operatorname{Im} \lambda=\sum_{l=1}^{\infty} \frac{1}{l !} \frac{\partial^{l} \lambda_{r}}{\partial z^{l}}(\xi, z(\xi ; k)) \operatorname{Im}\{(\operatorname{Re} z(\xi ; \lambda)-z(\xi ; k))+i \operatorname{Im} z(\xi ; \lambda)\}^{l}$

puisque $\lambda(\xi, \sigma)$ et $z(\xi ; k)$ prennent des valeurs réelles.

Pour $\lambda$ non réel, on déduit de là

$$
\begin{aligned}
& \frac{\operatorname{Im} \lambda}{\operatorname{Im} z(\xi ; \lambda)}=\frac{\partial \lambda_{r}}{\partial z}(\xi, z(\xi ; k))+(\operatorname{Re} z(\xi ; \lambda)-z(\xi ; k)) \frac{\partial^{2} \lambda_{r}}{\partial z^{2}}(\xi, z(\xi ; k)) \\
& \quad+\frac{1}{3 !}\left\{3(\operatorname{Re} z(\xi ; \lambda)-z(\xi ; k))^{2}-(\operatorname{Im} z(\xi ; \lambda))^{2}\right\} \frac{\partial^{3} \lambda_{r}}{\partial z^{3}}(\xi, z(\xi ; k)) \\
& \quad+\cdots
\end{aligned}
$$

puisque $\operatorname{Im} z(\xi ; \lambda) \neq 0$. En faisant tendre $\operatorname{Im} \lambda$ vers zéro dans (2.8), on obtient (2.5).

Passons ensuite au cas où $\sigma^{0}$ est double. Dire que $\sigma^{0}$ est une racine caractéristique double réelle de la matrice $M\left(\xi^{0} ; k^{0}\right)$ équivaut aux conditions :

$$
\frac{\partial \lambda_{r}}{\partial \boldsymbol{z}}\left(\xi^{0}, \sigma^{0}\right)=0 \quad \text { et } \quad \frac{\partial^{2} \lambda_{r}}{\partial z^{2}}\left(\xi^{0}, \sigma^{0}\right) \neq 0 .
$$

Cela se montre de la même manière que le cas précédent. De (2.9) il existe, en vertu du théorème d'existence des fonctions implicites, une fonction analytique à valeurs réelle $\sigma=\sigma(\xi)$ et une seule, définie dans un voisinage de $\xi=\xi^{0}$ telle que

$$
\sigma^{0}=\sigma\left(\xi^{0}\right) \quad \text { et } \quad \frac{\partial \lambda_{r}}{\partial \boldsymbol{z}}(\xi, \sigma(\xi)) \equiv 0 .
$$

Au voisinage du point $z=\sigma(\xi)$, développons $\lambda_{r}(\xi, \tau)$ (considéré comme fonction de $z$ avec paramètres $\left.\xi=\left(\xi_{1}, \cdots, \xi_{n-1}\right)\right)$ en série de Taylor

$$
\lambda=\lambda_{r}(\xi, z)=\sum_{l=0}^{\infty} \frac{1}{l !} \frac{\partial^{l} \lambda_{r}}{\partial z^{l}}(\xi, \sigma(\xi))(z-\sigma(\xi))^{l} .
$$

A cause de (2.10), la fonction de $z: \lambda(\xi, z)-\lambda(\xi, \sigma(\xi))$ a un zéro 
d'ordre 2 à chaque point $z=\sigma(\xi)$. Donc il existe, en vertu du théorème des fonctions inverses dans la théorie des fonctions, une fonction $z=z(\xi ; \lambda)$ définie au voisinage de $\left(\xi^{0}, k^{0}\right)$, ayant les propriétés :

i) $z(\xi ; \lambda)$ vérifie

$$
\sigma^{0}=z\left(\xi^{0}, k^{0}\right) \text { et } \lambda \equiv \lambda_{r}(\xi, z(\xi ; \lambda)),
$$

en particulier

$$
\sigma(\xi) \equiv \lambda_{r}(\xi, z(\xi ; \sigma(\xi)))
$$

ii) $\lambda=\lambda_{r}(\xi, \sigma(\xi))$ est un point critique algébrique d'ordre 1 de la fonction $z(\xi ; \lambda)$ de $\lambda$ et $z(\xi ; \lambda)$ admet le développement en série de Puiseux :

$$
z-\sigma(\xi)=\sum_{l=1}^{\infty} \alpha_{l}(\xi)\left(\lambda-\lambda_{r}(\xi, \sigma(\xi))\right)^{l / 2}
$$

En particulier, on a

$$
z-\sigma^{0}=\sum_{l=1}^{\infty} \alpha_{l}\left(\xi^{0}\right)\left(\lambda-k^{0}\right)^{l / 2}
$$

Pour la fonction $z=\boldsymbol{z}(\xi ; \lambda)$ ainsi déterminée, il est évident que sa valeur $z(\xi ; \lambda)$ est une racine caractéristique de $M(\xi ; \lambda)$ pour chaque $(\xi, \lambda)$ voisin de $\left(\xi^{0}, k^{0}\right)$ et que $\sigma(\xi)$ est racine caractéristique double réelle de $M(\xi ; \lambda)$ avec $\lambda=\lambda_{r}(\xi, \sigma(\xi))$.

Dans ce cas, si l'on pose

$$
\lambda=\lambda_{r}(\xi, \sigma(\xi)) \pm i \varepsilon, \quad \varepsilon>0
$$

on a

$$
\lim _{\operatorname{Im} \lambda \rightarrow 0} \frac{\operatorname{Im} \lambda}{\operatorname{Im} z(\xi ; \lambda)}=0 .
$$

En effet, en substituant $z$ dans (2.10) par $z=z(\xi ; \lambda)$ et en prenant les parties imaginaires, on peut aisément le constater de la même façon que le cas précédent.

Nous remarquons maintenant la propriété suivante que l'on peut déduire de la propriété d'être hermitiques des $A_{j}, j=1, \cdots, n .^{3}$,

“Les racines caractéristiques de $M(\xi ; \lambda)$ sont les complexes

3) Si les $A_{j}$ sont des matrices à éléments réels, c'est aussi vrai. 
(*) conjugués de celles de $M(\xi ; \bar{\lambda}) . \quad \bar{\lambda}$ désigne ici le complexe conjugué de $\lambda$ ".

Soient $\xi^{0}$ un point quelconque de $\Xi^{n-1}, k^{0}$ un nombre réel $\neq 0$, et $z_{1}^{0}, \cdots, z_{2 m}^{0}$ les racines caractéristiques de la matrice $M\left(\xi^{0} ; k^{0}\right)$. Pour un choix convenable de $\left(\xi^{0}, k^{0}\right)$, il existe des racines réelles parmi eux. Dans un tel cas, appelons $z_{1}^{0}, \cdots, z_{2_{p}}^{0}$ ces racines réelles et $z_{2_{p^{+1}}}^{0}, \cdots, z_{2_{m}}^{0}$ les autres non réelles (si elles existent) puisque, d'après la remarque faite plus haut, le nombre des racines réelles et celui des racines non réelles sont tous les deux pairs, ${ }^{4)}$ chacune des racines étant comptée autant de fois que l'indique son ordre de multiplicité. Lorsque $M\left(\xi^{0}, k^{0}\right)$ admet des racines caractéristiques multiples réelles, il n'existe qu'un couple de racines doubles réelles par l'hypothèse L. 3). Dans ce cas on peut supposer que $z_{1}^{0}$ et $z_{p+1}^{0}\left(z_{1}^{0}=z_{p+1}^{0}\right)$ sont le couple de ces racines. Dans ce qui suit, nous le supposerons.

Soit $W=W\left(\xi^{0}\right)$ un petit voisinage de $\xi^{0}$ dans $\Xi^{n-1}$. D'autre part nous posons, pour $\delta>0$,

$$
\begin{array}{ll}
\text { (2. 16) } & \Lambda_{\delta}=\Lambda_{\delta}\left(k^{0}\right)=\left\{\lambda \in \boldsymbol{C} ;\left|\operatorname{Re} \lambda-k^{0}\right|<\delta,|\operatorname{Im} \lambda|<\delta\right\}, \\
(2.16)^{\prime} & \Lambda_{\delta}^{+}=\Lambda_{\delta}^{+}\left(k^{0}\right)=\left\{\lambda \in \boldsymbol{C} ;\left|\operatorname{Re} \lambda-k^{0}\right|<\delta, 0<\operatorname{Im} \lambda<\delta\right\}, \\
(2.16)^{\prime \prime} & \Lambda_{\delta}^{-}=\Lambda_{\delta}^{-}\left(k^{0}\right)=\left\{\lambda \in \boldsymbol{C} ;\left|\operatorname{Re} \lambda-k^{0}\right|<\delta,-\delta<\operatorname{Im} \lambda<0\right\} .
\end{array}
$$

D'après la discussion faite précédemment, on peut faire correspondre à chaque racine $z_{j}^{0}$ réelle la fonction $z=z_{j}(\xi ; \lambda)$ avec $z_{j}^{0}=z_{j}\left(\xi^{0} ; k^{0}\right)$ définie au voisinage de $\left(\xi^{0}, k^{0}\right)$ ayant les propriétées suivantes.

Cas où $z_{j}^{0}$ est racine simple réelle.

s. i) $z_{j}(\xi ; \lambda)$ est analytique en $\xi$ et $\lambda$ dans $W \times \Lambda_{\delta}$.

s. ii) $z_{j}(\xi ; \lambda)$ est racine simple de $\operatorname{dét}(z I-M(\xi ; \lambda))=0$.

s. iii) $\lim _{\operatorname{Im} \lambda \rightarrow 0} \frac{\operatorname{Im} \lambda}{\operatorname{Im} z_{j}(\xi ; \lambda)} \neq 0$.

Cas où $z_{j}^{0}$ est racine double réelle.

d. i) $z(\xi ; \lambda)$ est une fonction multiforme de $\lambda$ telle qu'elle admette le développement en série de Puiseux de la forme:

4) Dans cet article, nous utiliserons ce fait pour des raisons de convenance. Par suite, la relation entre les racines de $M(\xi ; \lambda)$ et celles de $M(\xi ; \bar{\lambda})$ ne jouera aucun rôle essentiel dans notre travail. 


$$
z(\xi ; \lambda)=\sum_{l=0}^{\infty} \alpha_{l}(\xi)(\lambda-\beta(\xi))^{l / 2}, k^{0}=\beta\left(\xi^{0}\right)
$$

où $\alpha_{l}(\xi)$ et $\beta(\xi)$ sont des fonctions analytiques des variables réelles $\xi=\left(\xi_{1}, \cdots, \xi_{n-1}\right)$, définies au voisinage de $\xi^{0}$ et de plus $\alpha_{0}(\xi)$ et $\beta(\xi)$ prennent des valeurs réelles.

d. ii) Pour $\lambda$ non réel, $z(\xi ; \lambda)$ est racine caractéristique simple de $M(\xi ; \lambda)$.

d. iii) $\lim _{\operatorname{Im} \lambda \rightarrow 0} \frac{\operatorname{Im} \lambda}{\operatorname{Im}} \frac{\ln }{z(\xi ; \lambda)}=0$.

Comme nous l'avons déjà remarqué dans le $\S 1$, à cause de l'hyperbolicité, toute fonction $z_{j}(\xi ; \lambda)$ ne prend jamais de valeurs réelles lorsque $\operatorname{Im} \lambda \neq 0$. Donc, si $z_{j}^{0}$ est racine simple, $\operatorname{Im} z_{j}(\xi ; \lambda)$ a le même signe (positif ou négatif) pour $\operatorname{Im} \lambda>0$. Il en est de même pour $\operatorname{Im} \lambda<0$.

Lorsque $z_{1}^{0}, \cdots, z_{2_{p}}^{0}$ sont toutes simples, on peut supposer que $\operatorname{Im} z_{j}(\xi ; \lambda)>0, j=1, \cdots, p$ et $\operatorname{Im} z_{j}(\xi ; \lambda)<0, j=p+1, \cdots, 2 p$, quand $\operatorname{Im} \lambda>0$ en faisant le réarrangement si nécessaire.

Alors on a nécessairement

$\operatorname{Im} z_{j}(\xi ; \lambda)<0, j=1, \cdots, p \quad$ et $\quad \operatorname{Im} z_{j}(\xi ; \lambda)>0, j=p+1, \cdots, 2 p$

quand $\operatorname{Im} \lambda<0$.

Considérons ensuite le cas où $z_{1}^{0}=z_{p+1}^{0}$ est racine double réelle. Alors les autres $z_{2}^{0}, \cdots, z_{p}^{0}, z_{p+2}^{0}, \cdots, z_{2 p}^{0}$ sont par hypothèse les racines simples réelles. Donc, pour les $z_{j}(\xi ; \lambda) j=2, \cdots, p, p+2, \cdots, 2 p$, on peut aussi faire la même supposition qu'au cas ci-dessus. D'autre part, la fonction $z(\xi ; \lambda)\left(\equiv z_{1}(\xi ; \lambda) \equiv z_{p^{+1}}(\xi ; \lambda)\right)$ correspondant à $z_{1}^{0}=z_{p+1}^{0}$ est multiforme par rapport à $\lambda$ et composée de branches uniformes distinctes $\operatorname{Im} z(\xi ; \lambda)>0$ et $\operatorname{Im} z(\xi ; \lambda)<0$ dans $\operatorname{Im} \lambda>0$ (plus précisément dans $\left.W \times \Lambda_{\delta}^{+}\right)$. Il en est de même pour $\operatorname{Im} \lambda<0$.

Dans ces considérations, définissons les fonctions $\tau=\tau_{j}^{+}(\xi ; \lambda)$ et $\tau=\tau_{j}^{-}(\xi ; \lambda), j=1, \cdots, p$, de la manière suivante (Désormais nous utiliserons la lettre $\tau$ au lieu de $z$ pour représenter une variable complexe).

Lorsque les racines réelles $z_{1}^{0}, \cdots, z_{2 p}^{0}$ sont toutes simples, posons pour $\lambda$ non réel 


$$
\begin{aligned}
& \text { (2.18) }\left\{\begin{array}{l}
\tau_{j}^{+}(\xi ; \lambda)=z_{j}(\xi ; \lambda) \\
\tau_{j}^{-}(\xi ; \lambda)=z_{p^{+j}}(\xi ; \lambda)
\end{array} \quad j=1, \cdots, p, \text { pour } \operatorname{Im} \lambda>0,\right. \\
& (2.18)^{\prime} \quad\left\{\begin{array}{l}
\tau_{j}^{+}(\xi ; \lambda)=z_{p^{+j}}(\xi ; \lambda) \\
\tau_{j}^{-}(\xi ; \lambda)=z_{j}(\xi ; \lambda)
\end{array} \quad j=1, \cdots, p, \quad \text { pour } \operatorname{Im} \lambda<0 .\right.
\end{aligned}
$$

Si $z_{1}^{0}=z_{p+1}^{0}$ est racine double réelle, nous prenons comme $\tau=\tau_{1}^{+}(\xi ; \lambda)$ pour $\operatorname{Im} \lambda>0$ (resp. $\operatorname{Im} \lambda<0$ ) la détermination de la fonction $z(\xi ; \lambda)$ avec $\operatorname{Im} z(\xi ; \lambda)>0$ et comme $\tau=\tau_{1}^{-}(\xi ; \lambda)$ pour $\operatorname{Im} \lambda>0($ resp. $\operatorname{Im} \lambda<0)$ la détermination de $z(\xi ; \lambda)$ avec $\operatorname{Im} z(\xi ; \lambda)<0$. Quant aux autres $\tau_{j}^{ \pm}(\xi ; \lambda), j=2, \cdots, p$, nous suivons la définition précédente : $(2.18)$ et (2.18)'. Dans tous les cas nous avons les $2 p$ fonctions $\tau=\tau_{j}^{ \pm}(\xi ; \lambda)$, $j=1, \cdots, p$, définies dans $W \times \Lambda_{\delta}^{ \pm} . \quad \tau=\tau_{j}^{ \pm}(\xi ; \lambda)$ sont analytiques dans $W \times \Lambda_{\delta}^{ \pm}$et peuvent être prolongées par continuité dans $W \times \bar{\Lambda}_{\delta}^{ \pm}$ respectivement, $\bar{\Lambda}_{\delta}^{ \pm}$désignant l'adhérence de $\Lambda_{\delta}^{ \pm}$dans $C$ respectivement. Nous considérerons désormais $\tau=\tau_{j}^{ \pm}(\xi ; \lambda)$ comme ainsi prolongées.

D'autre part, aux racines caractéristiques $z_{2_{p^{+1}}}^{0}, \cdots, z_{2_{m}}^{0}$ non réelles de $M\left(\xi^{0}, k^{0}\right)$, on peut faire correspondre les fonctions $z_{2 p^{+1}}(\xi ; \lambda), \cdots$, $z_{2 m}(\xi ; \lambda)$ ayant les propriétés :

c. i) Elles sont définies et continues en $(\xi, \lambda)$ dans $W \times \bar{\Lambda}_{\delta}$.

c. ii) Elles sont des racines caractéristiques de $M(\xi ; \lambda)$ telles que $\operatorname{Im} z_{j}(\xi ; \lambda) \neq 0$ pour $(\xi, \lambda) \in W \times \bar{\Lambda}_{\delta}$ et $z_{j}^{0}=\boldsymbol{z}_{j}\left(\xi^{0} ; k^{0}\right)$.

Puisque le nombre des $z_{j}(\xi ; \lambda)$ telles que $\operatorname{Im} z_{j}(\xi ; \lambda)>0$ dans $W \times \Lambda_{\delta}$ est égale à celui des $z_{j}(\xi ; \lambda)$ telles que $\operatorname{Im} z_{j}(\xi ; \lambda)<0$ dans $W \times \Lambda_{\delta}$, nous pouvons supposer sans diminuer la généralité le suivant.

$$
\begin{aligned}
& \operatorname{Im} z_{j}(\xi ; \lambda)>0, j=2 p+1, \cdots, p+m \text { et } \operatorname{Im} z_{j}(\xi ; \lambda)<0, \\
& j=p+m+1, \cdots, 2 m .
\end{aligned}
$$

Alors nous posons :

$$
\left\{\begin{array}{l}
\tau_{p+1}^{+}(\xi ; \lambda)=z_{2 p^{+1}}(\xi ; \lambda), \cdots, \tau_{m}^{+}(\xi ; \lambda)=z_{p^{+m}}(\xi ; \lambda) \\
\tau_{p+1}^{-}(\xi ; \lambda)=z_{p^{+m+1}}(\xi ; \lambda), \cdots, \tau_{m}^{-}(\xi ; \lambda)=z_{2 m}(\xi ; \lambda)
\end{array}\right.
$$

Nous avons ainsi défini les $2 \mathrm{~m}$ fonctions $\tau=\tau_{j}^{ \pm}(\xi ; \lambda), j=1, \cdots, m$, correspondant aux $2 \mathrm{~m}$ racines caractéristiques de $M\left(\xi^{0} ; k^{0}\right)$.

Concernant les racines caractéristiques $z_{1}^{0}, \cdots, z_{2 m}^{0}$ de $M\left(\xi^{0} ; k^{0}\right)$ nous considérerons souvent le cas suivant comme une situation générale. 
(**) “ $z_{1}^{0}=z_{p+1}^{0}$ est racine double réelle, $z_{2}^{0}, \cdots, z_{p}^{0}, z_{p_{+2}}^{0}, \cdots, z_{2_{p}}^{0}$ sont les racines simples réelles et $z_{2 p+1}^{0}, \cdots, z_{2 m}^{0}$ les racines non réelles”.

Alors il résulte de la définition des $\tau_{j}^{ \pm}(\xi ; \lambda)$ et de la remarque $(*)$ le suivant.

$$
\begin{aligned}
\tau_{1}^{+}\left(\xi^{0} ; k^{0}+i 0\right)=\tau_{1}^{+}\left(\xi^{0} ; k^{0}-i 0\right)=\tau_{1}^{-}\left(\xi^{0} ; k^{0} \pm i 0\right) & =z_{1}^{0}=z_{p+1}^{0}, \\
\tau_{j}^{+}\left(\xi^{0} ; k^{0}+i 0\right)=\tau_{j}^{-}\left(\xi^{0} ; k^{0}-i 0\right)=z_{j}^{0}, \quad j & =2, \cdots, p, \\
\tau_{j}^{-}\left(\xi^{0} ; k^{0}+i 0\right)=\tau_{j}^{+}\left(\xi^{0} ; k^{0}-i 0\right)=z_{p+j}^{0}, \quad j & =2, \cdots, p, \\
\tau_{p+j}^{+}\left(\xi^{0} ; k^{0} \pm i 0\right)=z_{p_{p^{+j}}} \text { et } \tau_{p+j}^{-}\left(\xi^{0} ; k^{0} \pm i 0\right) & =z_{p+m+j}^{0}, \\
j & =1, \cdots, m-p .
\end{aligned}
$$

Construction de bases continues dans les espaces propres positif et négatif $\boldsymbol{E}^{ \pm}(\xi ; \lambda)$.

Nous allons maintenant construire un système $\left\{\boldsymbol{h}_{j}^{+}(\xi ; \lambda)\right\}_{j=1}, \cdots, m$ de fonctions vectorielles à valeurs dans $\boldsymbol{C}^{2 m}$ correspondant à $\left\{\tau_{j}^{+}(\xi ; \lambda)\right\}_{j=1, \cdots, m}$ jouissant des propriétés suivantes.

b. i) Les $\boldsymbol{h}_{j}^{+}(\xi ; \lambda), j=1, \cdots, m$, sont définis, continus en $(\xi, \lambda)$ dans $W \times \bar{\Lambda}_{\delta}^{+}$(resp. $W \times \bar{\Lambda}_{\delta}^{-}$) et analytiques en.$(\xi, \lambda)$ dans $W \times \Lambda_{\delta}^{+}$(resp. $\left.W \times \Lambda_{\delta}^{-}\right)$et linéairement indépendants pour chaque $(\xi, \lambda) \in W \times \Lambda_{\delta}^{+}$ (resp. $W \times \Lambda_{\delta}^{-}$).

b. ii) $\boldsymbol{h}_{j}^{+}(\xi ; \lambda)$ est un vecteur propre ordinaire de $M(\xi ; \lambda)$ correspondant à $\tau_{j}^{+}(\xi ; \lambda)$ lorsque $1 \leqslant j \leqslant p$ et $(\xi, \lambda) \in W \times \Lambda_{\delta}^{+}$(resp. $\left.W \times \Lambda_{\delta}^{-}\right)$.

b. iii) Le système $\left\{\boldsymbol{h}_{j}^{+}(\xi ; \lambda)\right\}_{j=1, \cdots, m}$ est une base de $E^{+}(\xi ; \lambda)$ pour chaque $(\xi, \lambda) \in W \times \Lambda_{\delta}^{+}$(resp. $\left.W \times \Lambda_{\delta}^{-}\right)$.

Définissons d'abord les $\boldsymbol{h}_{j}^{+}(\xi ; \lambda), j=p+1, \cdots, m$ correspondant aux $\tau_{j}^{+}(\xi ; \lambda), j=p+1, \cdots, m$. Pour cela prenons une base $\boldsymbol{h}_{p+1}^{0}, \cdots, \boldsymbol{h}_{m}^{0}$ dans le sous-espace $E^{+}\left(\xi^{0}, k^{0}\right)$ de $C^{2 m}$, engendré par les vecteurs propres ordinaires et généralisés pour les valeurs propres $\tau_{j}^{+}\left(\xi^{0} ; k^{0}+\mathrm{i} 0\right)$ $\left(=\tau_{j}^{+}\left(\xi^{0}, k^{0}-i 0\right)\right), j=p+1, \cdots, m$, non réelles de $M\left(\xi^{0}, k^{0}\right)$.

Par exemple, on peut choisir comme cette base un système qui consiste en un certain nombre de chaînes de Jordan de vecteurs. Nous posons :

$$
\boldsymbol{h}_{j}^{+}(\xi ; \lambda)=\frac{1}{2 \pi i} \int_{\gamma^{+}}(\tau I-M(\xi ; \lambda))^{-1} \boldsymbol{h}_{j}^{0} d \tau, \quad j=p+1, \cdots, m,
$$

où $\gamma^{+}$est une courbe fermée de Jordan, contenue en entier dans le 
demi-plan supérieur $\boldsymbol{C}^{+}=\{\tau ; \operatorname{Im} \tau>0\}$ et qui entoure les racines $\tau_{p+1}^{+}(\xi ; \lambda), \cdots, \tau_{m}^{+}(\xi ; \lambda)$, mais aucune des autres racines, lorsque $(\xi, \lambda)$ varie dans $W \times \bar{\Lambda}_{\delta}^{+}$(resp. $W \times \bar{\Lambda}_{\delta}^{-}$).

D'autre part nous définissons le vecteur $\boldsymbol{h}_{j}^{+}(\xi ; \lambda)$ correspondant à $\tau_{j}^{+}(\xi ; \lambda)(1 \leqslant j \leqslant p)$ de la manière suivante.

L'identité évidente :

$$
(A(\xi, \tau)-\lambda I) \cdot{ }^{t} \operatorname{cof}(A(\xi, \tau)-\lambda I)=\operatorname{dét}\left(A_{n}\right) \cdot \operatorname{dét}(\tau I-M(\xi ; \lambda)) I
$$

montre que toute colonne $\neq 0$ de la matrice $\left.{ }^{t} \operatorname{cof}(A(\xi, \tau)-\lambda I)\right|_{\tau=\tau_{j}+(\xi ; \lambda)}$ est un vecteur annulé à droite ${ }^{4)}$ par la matrice $(A(\xi, \tau)-\lambda I)$ $\tau_{j}^{+}(\xi ; \lambda)$ de $M(\xi ; \lambda)$ puisque l'on a

$$
\tau I-M(\xi, \lambda)=A_{n}^{-1}(A(\xi, \tau)-\lambda I) .
$$

${ }^{t} \operatorname{cof} Q$ désigne la matrice adjointe réduite de la matrice $Q$, à savoir le transposé de la matrice des cofacteurs (ou compléments algébriques) des éléments de $Q$. Or le rang de la matrice $A\left(\xi^{0}, \tau_{j}^{+}\right.$ $\left.\left(\xi^{0} ; k^{0} \pm i 0\right)\right)-k^{0} I$ est $2 m-1$, grâce à l'hyperbolicité stricte de l'opérateur $L$. Donc il existe au moins un cofacteur $\neq 0$ parmi les cofacteurs des éléments de $A\left(\xi^{0}, \tau_{j}^{+}\left(\xi^{0} ; k^{0} \pm i 0\right)\right)-k^{0} I$. Soit $\boldsymbol{h}_{j}^{+}(\xi ; \lambda)$ la colonne de la matrice $A(\xi, \tau)-\lambda I$ contenant au point $\left(\xi^{0}, k^{0}\right)$ un tel cofacteur. On peut alors supposer que $\boldsymbol{h}_{j}^{+}(\xi ; \lambda)$ ne s'annule jamais lorsque $(\xi, \lambda)$ décrit $W \times \bar{\Lambda}_{\delta}^{+}\left(\right.$resp. $\left.W \times \bar{\Lambda}_{-}^{\delta}\right)$ en reprenant $W$ et $\delta$ plus petits si nécessaire. Les $\boldsymbol{h}_{j}^{+}(\xi ; \lambda), j=1, \cdots, p$ ainsi définis sont des fonctions continues de $(\xi, \lambda)$ dans $W \times \bar{\Lambda}_{\delta}^{+}$(resp. $W \times \bar{\Lambda}_{\delta}^{-}$) et analytiques dans $W \times \Lambda_{\delta}^{+}\left(\right.$resp. $\left.W \times \Lambda_{\delta}^{-}\right)$puisqu'elles sont obtenues en remplaçant $\sigma$ par $\tau_{j}^{+}(\xi ; \lambda)$ dans des polynômes en $\lambda$, $\xi$ et $\tau$.

Nous avons ainsi le système des matrices-colonnes de fonctions $\boldsymbol{h}_{j}^{+}(\xi ; \lambda), j=1, \cdots, m$, definies dans $W \times \bar{\Lambda}_{\delta}^{+}$(resp. $\left.W \times \bar{\Lambda}_{\delta}^{-}\right)$.

Il est aisé de voir que les $\boldsymbol{h}_{j}^{+}(\xi ; \lambda), j=1, \cdots, m$, sont linéairement indépendants pour chaque $(\xi, \lambda)$ dans $W \times \bar{\Lambda}_{\delta}^{+}\left(\right.$resp. $\left.W \times \bar{\Lambda}_{\delta}^{-}\right)$et constituent une base de $E^{+}(\xi ; \lambda)$ pour chaque $(\xi, \lambda) \in W \times \Lambda_{\delta}^{+}$(resp. $\left.W \times \Lambda_{\delta}^{-}\right)$.

De la même manière nous pouvons construire un système $\left\{\boldsymbol{h}_{j}^{-}(\xi ; \lambda)\right\}_{j=1, \ldots, m}$ de matrices-colonnes de fonctions correspondant à

5) On l'appelle "right nul vector" en anglais. 
$\left\{\tau_{j}^{-}(\xi ; \lambda)\right\}_{j=1, \cdots, m}$ qui jouit des propriétés correspondantes.

Désignons par $E^{ \pm}(\xi ; k+i 0)$ (resp. $\left.E^{ \pm}(\xi ; k-i 0)\right)$ les sous-espaces de $\boldsymbol{C}^{2 m}$, à $m$ dimensions, engendrés par les vecteurs $\boldsymbol{h}_{j}^{ \pm}(\xi ; k+i 0)$, $j=1, \cdots, m$ (resp. $\left.\boldsymbol{h}_{j}^{ \pm}(\xi ; k-i 0), j=1, \cdots, m\right)$ linéairements indépendants respectivement. On a alors

$$
E^{ \pm}(\xi ; k) \subset E^{ \pm}(\xi ; k+i 0) \quad \text { et } \quad E^{ \pm}(\xi ; k) \subset E^{ \pm}(\xi ; k-i 0)
$$

respectivement.

Lorsque la matrice $M\left(\xi^{0} ; k^{0}\right)$ admet un couple de racines caractéristiques doubles réelles, on a par notre convention

$$
\tau_{1}^{+}\left(\xi^{0} ; k^{0} \pm i 0\right)=\tau_{1}^{-}\left(\xi^{0} ; k^{0} \pm i 0\right)
$$

et donc d'après la construction

$$
\boldsymbol{h}_{\mathbf{1}}^{+}\left(\xi^{0} ; k^{0} \pm i 0\right)=\boldsymbol{h}_{\mathbf{1}}^{-}\left(\xi^{0} ; k^{0} \pm i 0\right) .
$$

En général, cette relation est valable pour tout $(\xi, k)$ tel que $M(\xi, k)$ admette un cour'e de racines caractéristiques doubles reélles.

Nous définissons maintenant, en utilisant les vecteurs $\boldsymbol{h}_{j}^{+}(\xi ; \lambda)$, $j=1, \cdots, m$, définis plus haut, le déterminant de Lopatinski par

$$
\Delta(\xi ; \lambda)=\operatorname{dét}\left(\left\langle\boldsymbol{b}_{i}, \boldsymbol{h}_{j}^{+}(\xi ; \lambda)\right\rangle\right)
$$

où $\boldsymbol{b}_{i}$ désigne la $i$-ième colonne de la matrice $B$ et $\langle\boldsymbol{h}, \boldsymbol{g}\rangle$ le produit scalaire réel $\boldsymbol{h} \cdot \boldsymbol{g}=\sum_{l} h_{l} g_{l}$. On le définit localement, mais on peut le définir globalement dans $\boldsymbol{\Xi}^{n-1} \times \overline{\boldsymbol{C}}^{+}-\{(0,0)\} \quad$ (resp. $\boldsymbol{\Xi}^{n-1} \times \overline{\boldsymbol{C}}^{-}-$ $\{(0,0)\})$ à l'aide d'une partition $C^{\infty}$ de l'unité dans $(\xi, \lambda)$-espace.

\section{$\S 3$. Quelques Lemmes}

Considérons un système d'équations différentielles ordinaires à paramètres $\xi=\left(\xi_{1}, \cdots, \xi_{n-1}\right)$ et $\lambda$

$$
\left(\frac{1}{i} \frac{d}{d x_{n}}-M(\xi ; \lambda)\right) U\left(x_{n}, \xi ; \lambda\right)=0 \quad x_{n}>0,
$$

avec la condition

$$
B U(0, \xi ; \lambda)=\boldsymbol{g} \in \boldsymbol{C}^{\boldsymbol{l}} .
$$

Ici $M(\xi ; \lambda)=A_{n}^{-1}\left(\lambda I-A^{\prime}(\xi)\right)$ et $B$ est une matrice de type $(l, 2 m)$ à éléments constants, de rang $l$. 
Nous nous proposons le problème suivant pour $\lambda$ non réel.

"Chercher les conditions qu'il faut imposer à $B$ pour que quel que soit $\boldsymbol{g} \in \boldsymbol{C}^{l}$, il existe une solution unique à croissance lente (ou dans $L^{2}\left(\boldsymbol{R}_{+}^{1}\right)$ ) du problème (3.1)-(3.2)."

En tenant compte de ce que la matrice $M(\xi ; \lambda)$ n'admet pas de racines caractéristiques réelles pour $\lambda$ non réel, une solution $U\left(x_{n}, \xi ; \lambda\right)\left(x_{n}>0\right)$ de (3.1) est à croissance lente si et seulement si $U(0, \xi ; \lambda) \in E^{+}(\xi ; \lambda)$. Notre problème revient donc à trouver les conditions sur $B$ telles que pour tout $\boldsymbol{g} \in \boldsymbol{C}^{l}$ il existe une solution unique $U(0, \xi ; \lambda)$ dans l'espace propre positif $E^{+}(\xi ; \lambda)$, de l'équation linéaire $B U(0, \xi ; \lambda)=\boldsymbol{g}$.

Lemme 3.1 (Hersh. [3]) Soient $\lambda$ non réel et $\xi \in \Xi^{n-1}$. Alors les propositions suivantes sont équivalentes.

$1^{\circ}$ Quel que soit $\boldsymbol{g} \in \boldsymbol{C}^{l}$, il existe une solution unique $U\left(x_{n}, \xi ; \lambda\right)$ $\left(x_{n}>0\right) \dot{a}$ croissance lente (ou dans $L^{2}\left(\boldsymbol{R}_{+}^{1}\right)$ ) du probleme (3. 1)-(3.2).

$2^{\circ} \quad$ L'opérateur linéaire $B$ limité sur $E^{+}(\xi ; \lambda)$ est biunivoque de $E^{+}(\xi ; \lambda)$ sur $C^{l}$. (Il'en découle $\left.l=m\left(=\operatorname{dim} E^{+}(\xi ; \lambda)\right)\right)$.

$3^{\circ}$ i) $l=m$ et ii) $\mathscr{B}(=\operatorname{ker} B) \cap E^{+}(\xi ; \lambda)=\{0\}$, autrement dit, $\mathscr{B} \oplus E^{+}(\xi ; \lambda)=\boldsymbol{C}^{2 m}$.

$4^{\circ} \quad \Delta(\xi ; \lambda)=\operatorname{dét}\left(\left\langle\boldsymbol{b}_{i}, \boldsymbol{h}_{j}^{+}(\xi ; \lambda)\right\rangle\right) \neq 0$.

Lemme 3. 2. Soient $\lambda \in \boldsymbol{C}, \xi \in \mathbb{G}^{n-1}$ et $U\left(x_{n}, \xi ; \lambda\right), V\left(x_{n}, \xi ; \lambda\right)$ deux solutions quelconques de l'équation (3.1). Si l'intégrale

$$
\int_{0}^{\infty} U\left(x_{n}, \xi ; \lambda\right) \cdot \overline{V\left(x_{n}, \xi ; \lambda\right)} d x_{n}^{6}
$$

est convergente, ${ }^{7)}$ on a alors

(3. 4) $A_{n} U(0, \xi ; \lambda) \cdot \overline{V(0, \xi ; \lambda)}=2(\operatorname{Im} \lambda) \int_{0}^{\infty} U\left(x_{n}, \xi ; \lambda\right) \cdot \overline{V\left(x_{n}, \xi ; \lambda\right)} d x_{n}$.

Démonstration. Nous écrivons, pour simplifier $U\left(x_{n}\right)$ au lieu de $U\left(x_{n}, \xi ; \lambda\right)$ et $V\left(x_{n}\right)$ au lieu de $V\left(x_{n}, \xi ; \lambda\right)$.

Considérons l'identité évidente :

6) $U \cdot \bar{V}$ désigne le produit scalaire $\langle U, \bar{V}\rangle=\sum_{l=1}^{2 m} U_{\iota} \bar{V}_{\iota}$.

7) Par exemple $U\left(x_{n}, \xi ; \lambda\right)=0\left(x_{n}^{\alpha}\right), V\left(x_{n}, \xi ; \lambda\right)=0\left(x_{n} \beta e^{-\gamma x_{n}}\right.$; quand $x \rightarrow \infty$, $r$ étant un nombre positif, $\alpha, \beta$ deux entiers $\geqslant 0$. 
$2 i(\operatorname{Im} \lambda) \int_{0}^{\infty} U\left(x_{n}\right) \cdot \overline{V\left(x_{n}\right)} d x_{n}=\int_{0}^{\infty} \lambda U\left(x_{n}\right) \cdot \overline{V\left(x_{n}\right)} d x_{n}-\int_{0}^{\infty} U\left(x_{n}\right) \cdot \overline{V\left(x_{n}\right)} d x_{n}$.

En remplaçant dans le second membre $\lambda U\left(x_{n}\right)$ et $\lambda V\left(x_{n}\right)$ par

$$
\begin{aligned}
& \lambda U\left(x_{n}\right)=\frac{1}{i} \frac{d}{d x_{n}} A_{n} U\left(x_{n}\right)+\left(\sum_{j=1}^{n-1} \xi_{j} A_{j}\right) U\left(x_{n}\right) \text { et } \\
& \lambda V\left(x_{n}\right)=\frac{1}{i} \frac{d}{d x_{n}} A_{n} V\left(x_{n}\right)+\left(\sum_{j=1}^{n-1} \xi_{j} A_{j}\right) V\left(x_{n}\right)
\end{aligned}
$$

respectivement, il vient

$$
=\frac{1}{i}\left\{\int_{0}^{\infty} \frac{d}{d x_{n}} A_{n} U\left(x_{n}\right) \cdot \overline{V\left(x_{n}\right)} d x_{n}+\int_{0}^{\infty} U\left(x_{n}\right) \cdot \frac{\bar{d}}{d x_{n}} A_{n} V\left(x_{n}\right) d x_{n}\right\}
$$

puisque la matrice $\sum_{j=1}^{n-1} \xi_{j} A_{j}$ est hermitique.

Par hypothèse, $U\left(x_{n}\right) \cdot \overline{V\left(x_{n}\right)}$ tend vers zéro quand $x_{n} \rightarrow \infty$ et $A_{n}$ est hermitique. Donc on obtient (3.4) par une intégration par parties.

Lemme 3.3. Supposons que la condition $B$ aux limites soit conservative pour l'opérateur $L=I \frac{\partial}{\partial t}-i A, c$ 'est-à-dire que $A_{n} \zeta \cdot \bar{\zeta}=0$ pour tout $\zeta \in \mathscr{B}=\operatorname{ker} B$. Alors on a, pour $\lambda$ non réel et pour tout vecteur réel $\xi \in \in \mathbb{\Xi}^{n-1}$,

$$
\mathscr{B} \cap E^{+}(\xi ; \lambda)=\{0\}, \quad \operatorname{donc} \Delta(\xi ; \lambda) \neq 0 .
$$

Démonstration. On fixe $\lambda$ non réel et $\xi \in \xi^{n-1}$. Soit $\boldsymbol{h}$ un vecteur quelconque non zéro dans $E^{+}(\xi ; \lambda)$. Si on considère la solution $U\left(x_{n}\right), x_{n} \geqslant 0$, du système d'équations (3.1) vérifiant la condition initiale $U(0)=\boldsymbol{h}$, on a

$$
U\left(x_{n}\right) \neq 0 \text { et } U\left(x_{n}\right)=0\left(e^{-\gamma x_{n}}\right) \text { quand } \quad x_{n} \rightarrow+\infty,
$$

où $\gamma$ est une constante positive. Donc on peut prendre cette fonction $U\left(x_{n}\right)$ comme $U$ et $V$ dans la formule (3.4) et alors on a

$$
A_{n} \boldsymbol{h} \cdot \overline{\boldsymbol{h}}=2(\operatorname{Im} \lambda) \int_{0}^{\infty}\left|U\left(x_{n}\right)\right|^{2} d x_{n} \neq 0,
$$

ce qui implique $\boldsymbol{h} \notin \mathscr{B}$. D'où on a $\mathscr{B} \cap E^{+}(\xi ; \lambda)=\{0\}$.

Remarque. Si $B$ est dissipatif pour $L$, à savoir si $A_{n} \zeta \cdot \bar{\zeta} \geqslant 0$ pour tout $\zeta \in \mathscr{B}$, on a $\mathscr{B} \cap E^{+}(\xi ; \lambda)=\{0\}$ pour $\forall \xi \in \Xi^{n-1}$ et pour tout $\lambda$ avec $\operatorname{Im} \lambda<0$. 
Soient $\xi$ un point quelconque de $g^{n-1}$ et $k$ un nombre réel $\xi$ et $k$ non tous deux nuls. Nous nous plaçons maintenant la situation (**) concernant les racines caractéristiques de $M(\xi ; \lambda)$ :

" $\tau_{1}^{+}(\xi ; k+i 0)=\tau_{1}^{+}(\xi ; k-i 0)$ est racine double réelle, $\tau_{2}^{+}(\xi ; k+i 0)$, $\cdots \tau_{p}^{+}(\xi ; k+i 0), \tau_{2}^{+}(\xi ; k-i 0), \cdots, \tau_{p}^{+}(\xi ; k-i 0)$ les racines simples réelles et $\quad \tau_{p+1}^{+}(\xi ; k+i 0)=\tau_{p+1}^{+}(\xi ; k-i 0), \cdots, \tau_{m}^{+}(\xi ; k+i 0)=\tau_{m}^{+}(\xi ; k-i 0) \quad$ les racines non réelles à partie imaginaire $>0$."

Nous avons alors le

\section{Lemme 3. 4.}

1) $E^{+}(\xi ; k) \ni \boldsymbol{h}, \overline{\boldsymbol{g}} \Rightarrow A_{n} \boldsymbol{h} \cdot \overline{\boldsymbol{g}}=0$

2) $E^{\top}(\xi ; k) \ni \boldsymbol{h}$ et $E^{0}(\xi ; k) \ni \overline{\boldsymbol{g}} \Rightarrow A_{n} \boldsymbol{h} \cdot \overline{\boldsymbol{g}}=0$

$E^{0}(\xi ; k)$ désigne ici le sous-espace de $\boldsymbol{C}^{2 m}$ engendré par les vecteurs propres ordinaires et généralisés correspondant aux valeurs propres réelles de $M(\xi ; k)$.

3)

i) $\quad A_{n} \boldsymbol{h}_{j}^{+}(\xi ; k \pm i 0) \cdot \overline{\boldsymbol{h}_{l}^{+}(\xi ; k \pm i 0)}=0, \quad j, l=1,2, \cdots, m$ et $j \neq l$.

ii) Pour le vecteur $\boldsymbol{h}_{1}^{+}(\xi ; k \pm i 0)$ correspondant à la racine double réelle $\tau_{1}^{+}(\xi ; k \pm i 0)$, on a

$$
A_{n} \boldsymbol{h}_{1}^{+}(\xi ; k \pm i 0) \cdot \overline{\boldsymbol{h}_{1}^{+}(\xi ; k \pm i 0)}=0 .
$$

iii) Pour le vecteur $\boldsymbol{h}_{j}^{+}(\xi ; k \pm i 0)$ correspondant à la racine simple réel $\tau_{j}^{+}(\xi ; k \pm i 0)$ respectivement, on a

$$
\begin{aligned}
& \left.A_{n} \boldsymbol{h}_{j}^{+}(\xi ; k+i 0) \cdot \overline{\boldsymbol{h}_{j}^{+}(\xi ; k+i 0}\right)>0, \\
& A_{n} \boldsymbol{h}_{j}^{+}(\xi ; k-i 0) \cdot \overline{\boldsymbol{h}_{j}^{+}(\xi ; k-i 0)}<0 .
\end{aligned} \quad j=2, \cdots, p .
$$

Démonstration. On fixe $k$ et $\xi$ non tous deux nuls.

1) et 2): Soient $\boldsymbol{h} \in E^{+}(\xi ; k)$ et $\boldsymbol{g} \in E^{+}(\xi ; k)$ ou $E^{0}(\xi ; k)$. Soient $U\left(x_{n}\right)$ et $V\left(x_{n}\right)$ deux solutions de (3.1) vérifiant $U(0)=\boldsymbol{h}$ et $V(0)=\boldsymbol{g}$ respectivement. Alors l'intégrale (3.3) est convergente. Donc on a, en vertu de la formule (3.4), $A_{n} \boldsymbol{h} \cdot \bar{g}=0$.

$3)$ : Soit $\boldsymbol{h}_{j}^{+}(\xi ; \lambda)$ le vecteur correspondant à $\tau_{j}^{+}(\xi ; \lambda)$, défini dans le $\S 2$. En se rappelant que si $\lambda$ est non réel et si $1 \leqslant j \leqslant p, \boldsymbol{h}_{j}^{+}(\xi ; \lambda)$ est un vecteur propre pour $\tau_{j}^{+}(\xi ; \lambda)$ de $M(\xi ; \lambda)$, la solution unique $U\left(x_{n}\right), x_{n} \geqslant 0$ de $(3.1)$ avec la condition initiale $U(0)=\boldsymbol{h}_{j}^{+}(\xi ; \lambda)$ est donnée par 


$$
U_{j}\left(x_{n}\right)=e^{i \tau_{j}+(\xi ; \lambda) x_{n}} \boldsymbol{h}_{j}^{+}(\xi ; \lambda) \quad(1 \leqslant j \leqslant p) .
$$

En appliquant la formule (3.4) aux $U_{j}$ et $U_{l}(1 \leqslant j, l \leqslant p)$, on a pour $\lambda$ non réel

$$
\begin{array}{r}
A_{n} \boldsymbol{h}_{j}^{+}(\xi ; \lambda) \cdot \overline{\boldsymbol{h}_{l}^{+}(\xi ; \lambda)}=\frac{2 i \operatorname{Im} \lambda}{\tau_{j}^{+}(\xi ; \lambda)-\overline{\tau_{l}^{+}(\xi ; \lambda)}} \boldsymbol{h}_{j}^{+}(\xi ; \lambda) \cdot \overline{\boldsymbol{h}_{l}^{+}(\xi ; \lambda)}, \\
j, l=1, \cdots, p .
\end{array}
$$

On pose ici $\lambda=k \pm i \varepsilon, \varepsilon>0$. En notant que si $j \neq l, \tau_{j}^{+}(\xi ; k \pm i 0)-$ $\tau_{l}^{+}(\xi ; k \pm i 0) \neq 0(|\xi|+|k| \neq 0)$ et en faisant tendre $\operatorname{Im} \lambda= \pm \varepsilon$ vers zéro, nous avons (i). Lorsque $j=l$, (3.5) prend en particulier la forme :

$$
\begin{gathered}
A_{s} \boldsymbol{h}_{j}^{+}(\xi ; k \pm i \varepsilon) \cdot \overline{\boldsymbol{h}_{j}^{+}(\xi ; k \pm i \varepsilon)}=\frac{ \pm \varepsilon}{\operatorname{Im} \tau_{j}^{+}(\xi ; k \pm i \varepsilon)}\left|\boldsymbol{h}_{j}^{+}(\xi ; k \pm i \varepsilon)\right|^{2} \\
j=2, \cdots, p .
\end{gathered}
$$

En faisant tendre $\varepsilon$ vers zéro dans cette formule, on obtient pour $j=1$ l'assertion ii) d'après d. iii) du $\S 2: \lim _{\varepsilon \downarrow 0} \pm \varepsilon / \operatorname{Im} \tau_{1}^{+}(\xi ; k+i \varepsilon)=0$, et pour $j=2, \cdots, p$ l'assertion iii) d'après s. iii) du $\$ 2$ : $\lim _{\varepsilon \downarrow 0} \varepsilon / \operatorname{Im} \tau_{j}^{+}(\xi ; k+i \varepsilon)>0$ et $\lim _{\varepsilon \downarrow 0}-\varepsilon / \operatorname{Im} \tau_{j}^{+}(\xi ; k-i \varepsilon)<0$. c. q. f. d.

Lemme 3. 5. Supposons que l'opérateur $L$ vérifie les conditions L. 1)-L. 3) et que B vérifie B. 1) et B. 2). Supposons aussi que $\xi \in \Xi^{n-1}$ et $k$ réel soient non tous deux nuls. Alors le déterminant de Lopatinski $\Delta(\xi ; k+i 0)=\operatorname{dét}\left(\left\langle\boldsymbol{b}_{i}, \boldsymbol{h}_{j}^{+}(\xi ; k+i 0)\right\rangle\right)($ resp. $\Delta(\xi ; k-i 0))$ peut s'annuler seulement pour $(\xi, k)$ tel que la matrice $M(\xi ; k)$ admette des racines caractéristiques doubles réelles (par hypothèse un seul couple de racines doubles réells).

Démonstration. Envisageons seulement le cas de $\Delta(\xi ; k+i 0)$ parce que l'oa peut raisonner le cas de $\Delta(\xi, k-i 0)$ de la même manière. On raisonne par l'absurde. Soient $\xi$ un vecteur dans $\Xi^{n-1}$ et $k$ réel $(|\xi|+|k| \neq 0)$ tels que les racines caractéristiques de $M(\xi ; k)$ soient toutes non réelles ou les racines caractéristiques réelles soient toutes simples. Supposons que $\Delta(\xi ; k+i 0)=0$. Il existe alors des constantes $C_{1}, \cdots, C_{m}$, non toutes nulles, telles que $\sum_{j=1}^{m} C_{j}\left\langle\boldsymbol{b}_{i}, \boldsymbol{h}_{j}^{+}(\xi ; k+i 0)\right\rangle=0, i=1, \cdots, m$. Cela signifie que $\sum_{j=1}^{m} C_{j} \boldsymbol{h}_{j}^{+}$ $(\xi ; k+i 0) \in \mathscr{B}$. 
Considérons d'abord le cas où la matrice $M(\xi ; k)$ n'admet aucune racine caractéristique réelle. Dans ce cas, on a

Donc,

$$
\sum_{j=1}^{m} C_{j} \boldsymbol{h}_{j}^{+}(\xi ; k+i 0) \in E^{+}(\xi ; k+i 0)=E^{+}(\xi ; k) .
$$

$\sum_{j=1}^{m} C_{j} \boldsymbol{h}_{j}^{+}(\xi ; k+i 0)=0$ par l'hypothèse B. 2$): \mathscr{B} \cap E^{+}(\xi ; k)=\{0\}$.

Puisque les vecteurs $\boldsymbol{h}_{j}^{+}(\xi ; k+i 0), j=1, \cdots, m$ sont linéairement indépendants, on a de là $C_{1}=\cdots=C_{m}=0$, ce qui est absurde. Passons au cas où les racines réelles sont toutes simples. Appelons $\tau_{1}^{+}(\xi ; k \pm i 0), \cdots, \tau_{p}^{+}(\xi ; k \pm i 0) \quad$ ces racines. Comme le vecteur $\zeta=\sum_{j=1}^{m} C_{j} \boldsymbol{h}_{j}^{+}(\xi ; k+i 0)$ appartient à $\mathcal{B}$, et que $B$ est conservatif pour $L$, on a

$$
0=A_{n} \zeta \cdot \bar{\zeta}=\sum_{j, l=1}^{m} C_{j} \bar{C}_{l} A_{n} \boldsymbol{h}_{j}^{+}(\xi ; k+i 0) \cdot \overline{\boldsymbol{h}_{l}^{+}(\xi ; k+i 0)} .
$$

A cause des 1)-3) du lemma 3.4, il vient

$$
=\sum_{j=1}^{n}\left|C_{j}\right|^{2} A_{n} \boldsymbol{h}_{j}^{+}(\xi ; k+i 0) \cdot \overline{\boldsymbol{h}_{j}^{+}(\xi ; k+i 0)} .
$$

D'où on a $C_{1}=\cdots=C_{p}=0$, parce que sinon la dernière quantité est positive d'après iii) de 3 ) dans le lemme 3.4. On a ainsi

$$
\zeta=C_{p^{+1}} \boldsymbol{h}_{p+1}^{+}(\xi ; k+i 0)+\cdots+C_{m} \boldsymbol{h}_{m}^{+}(\xi ; k+i 0) \in \mathscr{B}
$$

et $\left|C_{p^{+1}}\right|+\cdots+\left|C_{m}\right| \neq 0$. D'autre part $\zeta \in E^{+}(\xi ; k)$. Donc $\zeta=0$ par l'hy pothèse B. 2). D'où $C_{p^{+1}}=\cdots=C_{m}=0$, ce qui est absurde. c. q. f. d.

Le lemme suivant est une généralisation légère de la remarque finale dans G. Strang [14].

Lemme 3.6. Soient $\tau_{1}, \cdots, \tau_{m}$ des nombres complexes $\dot{a}$ partie imaginaire positive, $\boldsymbol{h}_{1}, \cdots, \boldsymbol{h}_{m}$ des vecteurs dans $\boldsymbol{C}^{N}$ linéairement indépendants et $p_{1}(s), \cdots, p_{m}(s)$ des polynômes d'une variable réelle $s$. On pose:

$$
U(x)=\sum_{j=1}^{m} C_{j} e^{i \tau} p_{j}(s) \boldsymbol{h}_{j} .
$$

On a alors la majoration suivante: 


$$
\int_{0}^{\infty}|U(x)|^{2} d s \geqslant \gamma \sum_{j=1}^{m} q_{j j}\left(\frac{1}{2 \operatorname{Im} \tau_{j}}\right)\left|C_{j}\right|^{2},
$$

$\gamma$ est ici le minimum des valeurs propres de la matrice de Gram $\left(\boldsymbol{h}_{j} \cdot \ddot{\boldsymbol{h}}_{k}\right)$ et $q_{j k}(s)$ désigne le polynôme $q_{m_{j k}}(s)=\sum_{l=0}^{m_{j k}} a_{l}^{\prime j, k)} l ! s^{l+1}$ correspondant $\dot{a} p_{j}(s) \bar{p}_{k}(s)=\sum_{l=0}^{m_{j k}} a_{l}^{(j, k)} s^{l}$.

Démonstration. On a

$$
\int_{0}^{\infty}|U(x)|^{2} d s=\sum_{j, k=1}^{m n} C_{j} \bar{C}_{k}\left\{\int_{0}^{\infty} e^{i\left(\tau_{j}-\tau_{k}\right) s} p_{j}(s) \bar{p}_{k}(s) d s\right\} \boldsymbol{h}_{j} \cdot \overline{\boldsymbol{h}}_{k} .
$$

Posons ici

$$
\begin{aligned}
& S_{j k}=\int_{0}^{\infty} e^{i\left(\tau_{j}-\tau_{k}\right) s} p_{j}(s) \bar{p}_{k}(s) d s=q_{j k}\left(\frac{i}{\tau_{j}-\bar{\tau}_{k}}\right), \\
& T_{j k}=\boldsymbol{h}_{j} \cdot \overline{\boldsymbol{h}}_{k} \text { et } \quad H_{j k}=S_{j k} T_{j k} .
\end{aligned}
$$

Alors la matrice $T=\left(T_{j k}\right)$ est la matrice de Gram des vecteurs $\boldsymbol{h}_{1} ; \cdots, \boldsymbol{h}_{m}$ linéairement indépendants et donc définie positive. D'autre part, la matrice $S=\left(S_{j k}\right)$ est définie non négative, car on a pour tout $\zeta=\left(\zeta_{1}, \cdots, \zeta_{m}\right) \in \boldsymbol{C}^{m}$

$$
\sum_{j, k=1}^{m} q_{j k}\left(\frac{i}{\tau_{j}-\bar{\tau}_{k}}\right) \zeta_{j} \bar{\zeta}_{k}=\int_{0}^{\infty}\left|\sum_{j=1}^{m} e^{i \tau_{j} s} p_{j}(s) \zeta_{j}\right|^{2} d s .
$$

Le lemme 3.6 est alors une conséquence immédiate du théorème suivant du à I. Schur. ${ }^{8)}$

"Soient $S=\left(S_{j k}\right)$ et $T=\left(T_{j k}\right)$ deux matrices hermitiennes, définies non-négatives. Si l'on pose $H_{j k}=S_{j k} T_{j k}$, on a, pour tout $\zeta \in \boldsymbol{C}^{m}$,

$$
\sum_{j, k=1}^{m} H_{j k} \zeta_{j} \bar{\zeta}_{k} \geqslant \lambda_{\min }(T) \sum_{j=1}^{m} S_{j j}\left|\zeta_{j}\right|^{2}
$$

où $\lambda_{\text {min }}(T)$ désigne le minimum des valeurs propres de T."

Lemme 3. 7. Soient L l'opérateur (1.1) et B une matrice de type

8) Voir I. Schur, J. für Math. Bd. 140, 14 (1911) ou G. Polya und G. Szegö, Aufgaben und Lehrsätze aus der Analysis II, Springer-Verlag (1925 ou 1965), le problème 35 P. 106 et sa solution du P. 307. 
$(l, N)$ à éléments constants de rang $l$. Supposons que $B$ soit conservatif pour L. Alors pour la solution $U\left(x_{n}, \xi ; \lambda\right) \in L^{2}\left(\boldsymbol{R}_{+}^{1}\right) d u$ problème (3.1)-(3.2), on a la majoration suivante:

$$
(\operatorname{Im} \lambda)^{2} \int_{0}^{\infty}\left|U\left(x_{n}, \xi ; \lambda\right)\right|^{2} d x_{: s} \leqslant C|\boldsymbol{g}|^{2}
$$

où $C$ est une constante qui ne dépend pas de $(\xi, \lambda)$ quand $(\xi, \lambda)$ décrit un sous-ensemble relativement compact de ${\boldsymbol{g}^{n-1}}^{n} \times \boldsymbol{C}$.

Démonstration. Pour un vecteur $\boldsymbol{g}$ fixé de $\boldsymbol{C}^{l}$, on peut aisément construire une fonction vectorielle $v\left(x_{n}\right)$ vérifiant les conditions :

$$
B v(0)=\boldsymbol{C},
$$

(3.10) $\int_{0}^{\infty}\left|v\left(x_{n}\right)\right|^{2} d x_{n} \leqslant$ Const $|\boldsymbol{g}|^{2}$ et $\int_{0}^{\infty}\left|\frac{d v\left(x_{n}\right)}{d x_{n}}\right|^{2} d x_{n} \leqslant$ Const $|\boldsymbol{g}|^{2}$.

Posons ici

$$
\begin{aligned}
& V\left(x_{n}, \xi ; \lambda\right)=U\left(x_{n}, \xi ; \lambda\right)-v\left(x_{n}\right), \\
& \mathcal{L}=A_{n}\left(\frac{1}{i}-\frac{d}{d x_{n}}\right)-\left(\lambda I-\sum_{j=1}^{n-1} \xi_{j} A_{j}\right) .
\end{aligned}
$$

Alors $V\left(x_{n}, \xi ; \lambda\right)$ satisfait à l'équation

$$
\mathcal{L}\left[V\left(x_{n}, \xi ; \lambda\right)\right]=-\mathcal{L}\left[v\left(x_{n}\right)\right], x_{n}>0
$$

et la condition aux limites

$$
B V(0, \xi ; \lambda)=0 .
$$

Nous allons montrer que pour cette fonction $V\left(x_{n}, \xi ; \lambda\right)$, on a l'estimation

$$
(\operatorname{Im} \lambda)^{2} \int_{0}^{\infty}\left|V\left(x_{n}, \xi ; \lambda\right)\right|^{2} d x_{n} \leqslant \int_{0}^{\infty}\left|\mathcal{L}\left[v\left(x_{n}\right)\right]\right|^{2} d x_{n} .
$$

Pour cela, considérons l'identité évidente :

$$
\begin{aligned}
& 2 i(\operatorname{Im} \lambda) \int_{0}^{\infty}\left|V\left(x_{n}, \xi ; \lambda\right)\right|^{2} d x_{n} \\
& \quad=\int_{0}^{\infty} \lambda V\left(x_{n}, \xi ; \lambda\right) \cdot \overline{V\left(x_{n}, \xi ; \lambda\right)} d x_{n}-\int_{0}^{\infty} V\left(x_{n}, \xi ; \lambda\right) \cdot \overline{\lambda V\left(x_{n}, \xi ; \lambda\right)} d x_{n} .
\end{aligned}
$$

En remplaçant dans le second membre $\lambda V\left(x_{n}, \xi ; \lambda\right) \operatorname{par}\left(A_{n} \frac{1}{i} \frac{d}{d x_{n}}+\right.$ 
$\left.\sum_{j=1}^{n-1} \xi_{i} A_{j}\right) V\left(x_{n}, \xi ; \lambda\right)+\mathcal{L}\left[v\left(x_{n}\right)\right]$ et en intégrant par parties, on obtient

$$
\begin{aligned}
& 2 i(\operatorname{Im} \lambda) \int_{0}^{\infty}\left|V\left(x_{n}, \xi ; \lambda\right)\right|{ }^{2} d x_{n}=i A_{n} V(0, \xi ; \lambda) \cdot \overline{V(0, \xi ; \lambda)} \\
& \quad+\int_{0}^{\infty}\left\{\mathcal{L}\left[v\left(x_{n}\right)\right] \cdot \overline{V\left(x_{n}, \xi ; \lambda\right)}-V\left(x_{n}, \xi ; \lambda\right) \cdot \overline{\left.\mathcal{L}\left[v\left(x_{n}\right)\right]\right\}} d x_{n} .\right.
\end{aligned}
$$

Compte tenu de (3.14), le premier terme dans le second membre s'annule par hypothèse. En prenant la valeur absolue de deux membres et en appliquant l'inégalité de Schwarz au second membre, on a

$$
\begin{aligned}
& 2|\operatorname{Im} \lambda| \int_{0}^{\infty}\left|V\left(x_{n}, \xi ; \lambda\right)\right|^{2} d x_{n} \\
& \quad \leqslant 2\left\{\int_{0}^{\infty}\left|\mathcal{L}\left[v\left(x_{n}\right)\right]\right|^{2} d x_{n}\right\}^{1 / 2}\left\{\int_{0}^{\infty}\left|V\left(x_{n}, \xi ; \lambda\right)\right|^{2} d x_{n}\right\}^{1 / 2} .
\end{aligned}
$$

D'où, on déduit (3.15). De (3.10), (3.11), (3.12) et (3.15), on déduit aussi la majoration désirée (3.8). Alors on peut choisir, compte tenu de la forme $(3.12)$ de $\mathcal{L}$, une constante $C$ qui ne dépend pas de $(\xi, \lambda)$ quand $(\xi, \lambda)$ parcourt un relativement compact de $\boldsymbol{\Xi}^{n-1} \times \boldsymbol{C}$. c. q. f. d.

Nous pouvons maintenant établir le théorème suivant sur les zéros du déterminant de Lopatinski et leur ordre.

Théorème 3.1. Supposons que l'opérateur L vérifie les conditions L. 1)-L. 3) et que $B$ vérifie B. 1) et B. 2). Alors le déterminant de Lopatinski $\Delta(\xi ; \lambda)$ défini dans $\boldsymbol{\Xi}^{n-1} \times \overline{\boldsymbol{C}}^{+}-\{(0,0)\} \quad$ (resp. $\boldsymbol{\Xi}^{n-1} \times \overline{\boldsymbol{C}}^{-}-$ $\{(0,0\})$ peut s'annuler seulement pour $\xi \in \Xi^{n-1}$ et $\lambda=k$ réel tels que $M(\xi ; k)$ admette des racines caractéristiques doubles réelles (un seul couple par hypothèse). Soit $\left(\xi^{0}, k^{0}\right)$ un tel point. Alors $\Delta(\xi ; \lambda)$ admet dans un voisinage $W \times \bar{\Lambda}_{\delta}^{+}$(resp. $\left.W \times \bar{\Lambda}_{\delta}^{-}\right)$de $\left(\xi^{0}, k^{0}\right)$ l'expression de la forme:

$$
\Delta(\xi ; \lambda)=\sum_{l=0}^{\infty} a_{l}(\xi)(\lambda-b(\xi))^{l / 2}
$$

où les $a_{l}(\xi)$ et $b(\xi)$ sont des fonctions analytiques des variables $\xi=\left(\xi_{1}, \cdots, \xi_{n-1}\right)$ définies dans $W=W\left(\xi^{0}\right)$ et $b(\xi)^{9)}$ prend des valeurs

9) Soit $\beta(\xi)$ la fonction dans la formule (2.17). Alors $b(\xi) \equiv \beta(\xi)$. 
réelles. De plus on a

$$
\left|a_{0}\left(\xi^{0}\right)\right|+\left|a_{1}\left(\xi^{0}\right)\right| \neq 0 .
$$

Démonstration. La première assertion n'est autre que la combinaison des lemmes 3.3 et 3.5. Pour envisager le problème de l'expression (3.16), plaçons-nous dans la situation (**) du $\S 2$, c'est-à-dire (2.20) concernant les racines caractéristiques de $M\left(\xi^{0} ; k^{0}\right)$ et rappelons le procédé de la construction de la base $\left\{\boldsymbol{h}_{j}^{+}(\xi ; \lambda)\right\}_{j=1, \cdots, m}$. Si $p+1 \leqslant j \leqslant m, h_{j}^{+}(\xi ; \lambda)$ peut se prolonger d'après la définition (2.21) en une fonction analytique $\boldsymbol{h}_{j}(\xi ; \lambda)$ définie dans $W \times \Lambda_{\delta}$. Si $1 \leqslant j \leqslant m$, $\boldsymbol{h}_{j}^{+}(\xi ; \lambda)$ est obtenu en remplaçant $\tau$ par $\tau_{j}^{+}(\xi ; \lambda)$ dans une matricecolonne $\boldsymbol{q}_{j}(\xi, \tau, \lambda)$ à éléments polynômes de $(\xi, \tau, \lambda)$. Compte tenu de la définition des $\tau_{j}^{+}(\xi ; \lambda), j=1, \cdots, p$, posons

$$
\begin{aligned}
& \boldsymbol{h}_{1}(\xi ; \lambda)=\boldsymbol{q}(\xi, \boldsymbol{z}(\xi ; \lambda) ; \lambda)
\end{aligned}
$$

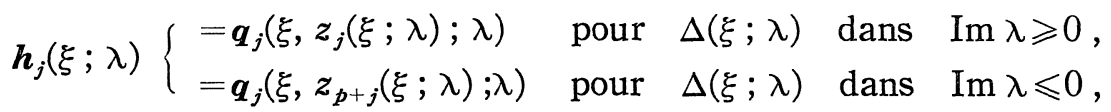

$$
\begin{aligned}
& j=2, \cdots, p \text {. }
\end{aligned}
$$

Alors on voit d'après s. i) et d. i) du $\S 2$ que dét $\left(\left\langle\boldsymbol{b}_{i}, \boldsymbol{h}_{j}(\xi ; \lambda)\right\rangle\right)$ est défini dans $W \times \Lambda_{\delta}$ et admet le développement en série de Puiseux de la forme:

$$
\sum_{l=0}^{\infty} a_{l}(\xi)(\lambda-b(\xi))^{l}
$$

où les $a_{l}(\xi)$ et $b(\xi)$ sont des fonctions analytiques réells de $\xi$, et $b(\xi)$ prend des valeurs réelles. D'où le déterminant de Lopatinski $\Delta(\xi ; \lambda)$ dans $W \times \bar{\Lambda}_{\delta}^{+}$(resp. $W \times \bar{\Lambda}_{\delta}^{-}$) admet l'expression (3. 16). Notre tâche est maintenant de montrer (3.17). Nous nous bornons ici à traiter $\Delta(\xi ; \lambda)$ dans $W \times \bar{\Lambda}_{\delta}^{+}$. On pourra raisonner de la même manière le cas de $\Delta(\xi ; \lambda)$ dans $W \times \bar{\Lambda}_{\delta}^{-}$. Considérons la matrice :

$$
\left(\begin{array}{ccc}
\left\langle\boldsymbol{b}_{1}, \boldsymbol{h}_{2}^{+}\left(\xi^{0} ; k^{0}+i 0\right)\right\rangle & \cdots\left\langle\boldsymbol{b}_{1}, \boldsymbol{h}_{m}^{+}\left(\xi^{0} ; k^{0}+i 0\right)\right\rangle \\
\vdots & \vdots \\
\left\langle\boldsymbol{b}_{\boldsymbol{m}}, \boldsymbol{h}_{2}^{+}\left(\xi^{0} ; k^{0}+i 0\right)\right\rangle & \cdots\left\langle\boldsymbol{b}_{m}, \boldsymbol{h}_{m}^{+}\left(\xi^{0} ; k^{0}+i 0\right)\right\rangle
\end{array}\right)
$$

Le rang de cette matrice est $m-1$. En effet si on suppose que le rang $<m-1$, il existe des constantes $C_{2}, \cdots, C_{m}$, non toutes nulles, telles que

$$
\zeta=C_{2} \boldsymbol{h}_{2}^{+}\left(\xi^{0} ; k^{0}+i 0\right)+\cdots+C_{m} \boldsymbol{h}_{m}^{+}\left(\xi^{0} ; k^{0}+i 0\right) \in \mathscr{B} .
$$


D'autre part on a, par hypothèse, $\mathscr{B} \cap E^{+}(\xi ; k)=\{0\}$ pour $\xi$ et $k$ non tous deux nuls. Alors on arrivera à une contradiction en raisonnant de la même manière que dans le lemme 3.5. Ainsi il existe un mineur $\neq 0$ d'ordre $m-1$ de la matrice (3.18). Par suite on trouve une fonction de $\varepsilon$ telle que

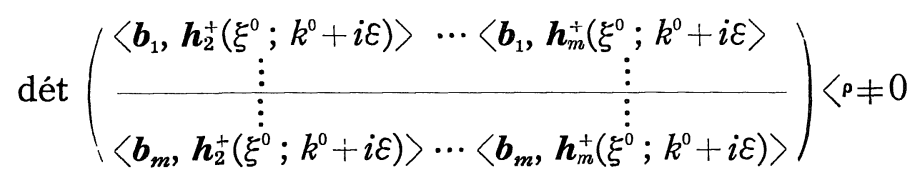

pour $\varepsilon \geqslant 0$ suffisamment petit, où le signe- $<\rho$ signifie la manque de la $\rho$-ième ligne. Comme la fonction est continue en $\varepsilon$, elle est en valeur absolue minorée par une constante positive qui ne dépend pas de $\varepsilon$, lorsque $\varepsilon$ décrit un petit intervalle $\left[0, \varepsilon^{0}\right]$. Ceci remarqué, considérons la solution ${ }^{t}\left(C_{1}(\varepsilon), \cdots, C_{m}(\varepsilon)\right)$ du système d'équations linéaires

$$
\left(\left\langle\boldsymbol{b}_{i}, \boldsymbol{h}_{j}^{+}\left(\xi^{0} ; k^{0}+i \varepsilon\right)\right\rangle\right)_{1 \leqslant i, j \leqslant m}\left(\begin{array}{c}
C_{1}(\varepsilon) \\
\vdots \\
C_{m}(\varepsilon)
\end{array}\right)=\left(\begin{array}{c}
0 \\
\vdots \\
1 \\
\vdots \\
0
\end{array}\right)(\rho
$$

pour $\varepsilon \in\left(0, \varepsilon^{0}\right]$. Alors on a pour $\varepsilon \in\left(0, \varepsilon^{0}\right]$

$$
C_{1}(\varepsilon)=\frac{(-1)^{i+\rho} \operatorname{dét}\left(\frac{\left\langle\boldsymbol{b}_{1}, \boldsymbol{h}_{2}^{+}\left(\xi^{0} ; k^{0}+i \varepsilon\right)\right\rangle \cdots\left\langle\boldsymbol{b}_{1}, \boldsymbol{h}_{m}^{+}\left(\xi^{0} ; k^{0}+i \varepsilon\right)\right\rangle}{\vdots} \begin{array}{c}
\vdots \\
\vdots \\
\left\langle\boldsymbol{b}_{m}, \boldsymbol{h}_{2}^{+}\left(\xi^{0} ; k^{0}+i \varepsilon\right)\right\rangle \cdots\left\langle\boldsymbol{b}_{m}, \boldsymbol{h}_{m}^{+}\left(\xi^{0} ; k^{0}+i \xi\right)\right\rangle
\end{array}\right)}{\Delta\left(\xi^{0} ; k^{0}+i \varepsilon\right)} .
$$

Soit $U\left(x_{n} ; \varepsilon\right), x_{n} \geqslant 0$ la solution de l'équation

$$
\left(\frac{1}{i} \frac{d}{d x_{n}}-M\left(\xi^{0} ; k^{0}+i \varepsilon\right)\right) U\left(x_{n} ; \varepsilon\right)=0, x_{n}>0
$$

avec la condition initiale

$$
U(0 ; \varepsilon)=\sum_{j=1}^{m} C_{j}(\varepsilon) \boldsymbol{h}_{j}^{+}\left(\xi^{0} ; k^{0}+i \varepsilon\right), \quad 0<\varepsilon \leqslant \varepsilon^{0} .
$$

En appliquant le lemme 3.7 à cette solution $U\left(x_{n} ; \varepsilon\right)$, on obtient

$$
\varepsilon^{2} \int_{0}^{\infty}\left|U\left(x_{n} ; \varepsilon\right)\right|^{2} d x_{n} \leqslant \text { Const , } \quad 0<\varepsilon \leqslant \varepsilon^{0} .
$$


où la constante ne dépend pas de $\varepsilon$. D'autre part la solution $U\left(x_{n} ; \varepsilon\right)$ s'exprime comme suit :

$$
\begin{aligned}
U\left(x_{n} ; \varepsilon\right)= & \sum_{j=1}^{p} C_{j}(\varepsilon) e^{i \tau_{j^{+}}\left(\xi^{0} ; k^{0}+i \varepsilon\right) x_{n}} \boldsymbol{h}_{j}^{+}\left(\xi^{0} ; k^{0}+i \varepsilon\right) \\
& +\sum_{j=p+1}^{m} C_{j}(\varepsilon) p_{j}\left(x_{n} ; \varepsilon\right) e^{i \tau_{j} j^{+}\left(\xi^{0} ; k^{0}+i \varepsilon\right) x_{n}} \boldsymbol{h}_{j}^{+}\left(\xi^{0} ; k^{0}+i \varepsilon\right),
\end{aligned}
$$

les $p_{j}\left(x_{n} ; \varepsilon\right), \quad j=p+1, \cdots, m$ étant des polynômes en $x$ à coefficients dépendant de $\varepsilon$, de degré $\leqslant m$. Par conséquent on peut appliquer le lemme 3.6 à $U\left(x_{n} ; \varepsilon\right)$. On a alors

$$
\begin{aligned}
\int_{0}^{\infty}\left|U\left(x_{n} ; \varepsilon\right)\right|^{2} d x_{n} \geqslant \gamma(\varepsilon)\left\{\sum_{j=1}^{n} \frac{\left|C_{j}(\varepsilon)\right|^{2}}{2 \operatorname{Im} \tau_{j}^{+}\left(\xi^{0} ; k^{0}+i \varepsilon\right)}\right. \\
\left.+\sum_{j=p+1}^{m} q_{j j}\left(\frac{1}{2 \operatorname{Im} \tau_{j}^{+}\left(\xi^{0} ; k^{0}+i \varepsilon\right)} ; \varepsilon\right)\left|C_{j}(\varepsilon)\right|^{2}\right\} .
\end{aligned}
$$

$\gamma(\varepsilon)$ est ici le minimum des valeurs propres de la matrice de Gram des vecteurs $\boldsymbol{h}_{j}^{+}\left(\xi^{0} ; k^{0}+i \varepsilon\right), j=1, \cdots, m$. Comme les $\boldsymbol{h}_{j}^{+}\left(\xi^{0} ; k^{0}+i \varepsilon\right)$ $j=1, \cdots, m$ sont continus en $\varepsilon$ dans $\left[0, \varepsilon^{0}\right]$ et linéairement indépendants pour chaque $\varepsilon \in\left[0, \varepsilon^{0}\right], \gamma(\varepsilon)$ est positif et minoré par une constante positive. On déduit de (3.19), (3.20), (3.21) et (3.23)

$$
\text { Const } \geqslant \varepsilon^{2} \gamma(\varepsilon)\left|C_{1}(\varepsilon)\right|^{2} / 2 \operatorname{Im} \tau_{1}^{+}\left(\xi^{0} ; k^{0}+i \varepsilon\right) \text {. }
$$

On a donc

$$
\text { Const } \geqslant \varepsilon^{2} d / \operatorname{Im} \tau_{1}^{+}\left(\xi^{0} ; k^{0}+i \varepsilon\right)\left|\Delta\left(\xi^{0} ; k^{0}+i \varepsilon\right)\right|^{2},
$$

$d$ étant une constante positive. De cette inégalité il résulte que $a_{0}\left(\xi^{0}\right)$ et $a_{1}\left(\xi^{0}\right)$ ne sont pas tous deux nuls. En effet, sinon on tira de l'expression (3.16)

$$
\left|\Delta\left(\xi^{0} ; k^{0}+i \varepsilon\right)\right|=o(\sqrt{\varepsilon}) \text { quand } \quad \varepsilon \downarrow 0 .
$$

Donc le second membre de (3.24) tend vers l'infini lorsque $\varepsilon$ tend vers zéro en restant positif, ce qui est absurde. On a ainsi (3.23).

$$
\text { c. q. f. d. }
$$

\section{$\S 4$. Solution Elémentaire de l'Opérateur $A-\lambda I$ dans l'Espace Libre $\boldsymbol{R}^{n}$}

Soit $A$ un opérateur différentiel matriciel de la forme: 


$$
A=\frac{1}{i} \sum_{j=1}^{n} A_{j} \frac{\partial}{\partial x_{j}}
$$

où les $A_{j}, j=1, \cdots, n$, sont des matrices à coefficients complexes $N \times N$. Dans la première partie de ce paragraphe, nous ne supposons pas nécessairement les $A_{j}, j=1, \cdots, n$, hermitiennes. De plus, nous imposons à $A$ les conditions suivantes plus générales que L. 1)-L. 3).

A. 1) Les racines caractéristiques de $A(\eta)=\sum_{j=1}^{n} \eta_{j} A_{j}$ sont toutes réelles et de plus leurs multiplicités sont invariantes pour tout vecteur réel $\eta=\left(\eta_{1}, \cdots, \eta_{n}\right) \neq 0$ :

$$
\begin{aligned}
& \operatorname{dét}(\lambda I-A(\eta))=\left(\lambda-\lambda_{1}(\eta)\right)^{m_{1}} \cdots(\lambda-\lambda(\eta))^{m_{l}} \text {, } \\
& m_{1}+\cdots+m_{l}=N \\
& \lambda_{1}(\eta)>\cdots>\lambda_{l}(\eta) \text { pour } \eta \neq 0 \text {. }
\end{aligned}
$$

A. 2) $A(\eta)$ est diagonalisable, en d'autres termes, semi-simple pour tout $\eta$.

A. 3) $\lambda_{j}(\eta)=0$ ou $\lambda_{j}(\eta) \neq 0$ pour tout $\eta \neq 0$.

Alors on a, pour $\lambda$ non réel,

$$
(A(\eta)-\lambda I)^{-1} \in \mathcal{S}_{\eta}^{\prime}
$$

où $S_{\eta}^{\prime}$ désigne l'espace des distributions tempérées sur $\boldsymbol{\Xi}^{n}$.

Nous posons pour $\lambda$ non réel

$$
E(x ; \lambda)=(2 \pi)^{-(n / 2)} \mathscr{F}^{-1}\left[(A(\eta)-\lambda I)^{-1}\right],
$$

$\mathscr{F}^{-1}$ désignant la transformation de Fourier inverse au sens des distributions. ${ }^{10)} E(x ; \lambda)$ est alors une solution élémentaire de l'opérateur $A-\lambda I$. En effet on a

$$
(A-\lambda I) E(x ; \lambda)=\delta(x) I,
$$

$\delta(x)$ étant la distribution de Dirac.

Notre premier but de ce paragraphe est d'établir le

Théorème 4.1. $E(x ; \lambda)$ est une fonction indéfiniment dérivable en $x$ dans $\boldsymbol{R}^{n}-\{0\}$ et dépend analytiquement de $\lambda$ dans $\boldsymbol{C}-\left\{l^{\prime}\right.$ axe

10) Si $\varphi \in L^{1}\left(\Xi^{n}\right), \mathscr{F}^{-1}[\varphi]$ est défini par la formule

$$
\mathscr{F}^{-1}[\varphi]=(2 \pi)^{-n / 2} \int_{\mathbb{\Xi}^{n}} e^{i<x, \eta>} \varphi(\eta) d \eta
$$


réel\}. Si on pose $\lambda=k \pm i \varepsilon, k$ réel et $\varepsilon>0$, pour tout indice multiple $\nu$ et pour $x \in \boldsymbol{R}^{n}-\{0\}$ et $k \neq 0$ arbitrairement fixés, les limites suivantes existent

$$
E^{(\nu)}(x ; k \pm i 0)=\lim _{\varepsilon \downarrow 0}\left(\frac{\partial}{\partial x}\right)^{\nu} E(x ; k \pm i \varepsilon)
$$

où la convergence est uniforme dans tout compact de $\boldsymbol{R}^{n}-\{0\}$. De plus $E^{(\nu)}(x ; k \pm i 0)$ sont des fonctions continue en $(x, k)$ dans le domaine $\left(\boldsymbol{R}^{n}-\{0\}\right) \times\left(\boldsymbol{R}^{1}-\{0\}\right){ }^{11}$

Pour démontrer ce théorème, nous préparons le

Lemme 4. 1. Soit $T_{\lambda}$ une distribution tempérée des variables $x=\left(x_{1}, \cdots, x_{n}\right)$, dépendant d'un paramètre $\lambda$ qui parcourt un domaine $\Lambda d u$ plan complexe $\boldsymbol{C}$, telle qu'on ait les propriétés suivantes:

i) Pour tout indice $\alpha$ multiple, $\left(\frac{\partial}{\partial \eta}\right)^{\alpha} \hat{T}_{\lambda}(\eta)^{12)}$ est une fonction continue de $(x, \lambda)$ dans $g^{n} \times \Lambda$.

ii) Pour tout $\eta$ fixé, $\hat{T}_{\lambda}(\eta)$ est une fonction analytique en $\lambda$.

iii) Pour tout $\alpha$, il existe une constante $C_{a}$ telle que

$$
\left|\left(\frac{\partial}{\partial \eta}\right)^{\infty} \hat{T}_{\lambda}(\eta)\right| \leqslant C_{\alpha}(1+|\eta|)^{l-|\alpha| d}
$$

où $d>0$ et $C_{a}$ ne dépend pas de $\lambda$.

On a alors les assertions:

$1^{\circ}$ Si on prend $\alpha$ tel que $|\alpha| \geqslant[(n+l+\rho) / d]+1^{13)}$ et si $|\nu| \leqslant \rho$ $(\partial / \partial x)^{\nu}\left(x^{\nu} T_{\lambda}\right)$ est une fonction continue de $(x, \lambda)$ dans $\boldsymbol{R}^{n} \times \Lambda$ et analytique en $\lambda$.

$2^{\circ}$ Pour tout $\nu,(\partial / \partial x)^{\nu} T_{\lambda}$ est une fonction continue de $(x, \lambda)$ dans $\left(\boldsymbol{R}^{n}-\{0\}\right) \times \Lambda$ et analytique en $\lambda$.

Preuve. $1^{\circ}$ D’après (4. 7) on a

11) Pour chaque $x \neq 0$ et fixé, on peut considérer $E(x ; \lambda)$ comme une fonction analytique de $\lambda$, définie dans le demi-plan supérieur $\operatorname{Im} \lambda>0$ ou dans le demi-plan inférieur $\operatorname{Im} \lambda<0$. Alors elle peut être prolongée analytiquement au delà de l'axe réel dans une partie de l'autre demi-plan. Mais nous n'avons pas besoin de ce fait dans cet article.

12) $\hat{T}_{\lambda}(\eta)$ désigne la transformée de Fourier de $T_{\lambda}$ par rapport à $x$.

13) $s$ étant un nombre réel, $[s]$ désigne le plus grand entier $\leqslant s$. 


$$
\left|\mathscr{F}\left[\left(\frac{\partial}{\partial x}\right)^{\nu}\left(x^{\alpha} T_{\lambda}\right)\right](\eta)\right|=\left|(i \eta)^{\nu}\left\{\left(i \frac{\partial}{\partial \eta}\right)^{\alpha} \hat{T}_{\lambda}(\eta)\right\}\right| \leqslant C_{\alpha}(1+|\eta|)^{l-|\alpha| d|-| \nu \mid} .
$$

Donc, si $|\alpha| \geqslant[(n+\rho+l) / d]+1$ et si $|\nu| \leqslant \rho,(\partial / \partial x)^{\nu}\left(x^{\alpha} T_{\lambda}\right)$ peut s'écrire sous forme d'une intégrale

$$
\left(\frac{\partial}{\partial x}\right)^{\nu}\left(x^{\infty} T_{\lambda}\right)=(2 \pi)^{-n / 2} \int_{\Xi^{n}} e^{i<x, \eta>}(i \eta)^{\nu}\left\{\left(i \frac{\partial}{\partial \eta}\right)^{\infty} \hat{T}_{\lambda}(\eta)\right\} d \eta
$$

D'où il suit que $(\partial / \partial x)^{\nu}\left(x^{\alpha} T_{\lambda}\right)$ est une fonction continue de $(x, \lambda)$. D'autre part on a, d'après la formule de Cauchy et (4.7),

$$
\left|\frac{\partial}{\partial \lambda}\left(\frac{\partial}{\partial \eta}\right)^{\infty} \hat{T}_{\lambda}(\eta)\right| \leqslant C_{\alpha}(1+|\eta|)^{l-|\alpha| d} .
$$

On déduit de (4.8) que $(\partial / \partial x)^{\nu}\left(x^{\alpha} T_{\lambda}\right)$ est analytique en $\lambda$.

$2^{\circ}$ Dans l'ouvert complémentaire de l'origine $x=0$, on a l'égalité évidente :

$$
\left(\frac{\partial}{\partial x}\right)^{\nu} T_{\lambda}=|x|^{-2 p}\left[|x|^{2 p}\left\{\left(\frac{\partial}{\partial x}\right)^{\nu} T_{\lambda}\right\}\right] .
$$

Or, la condition (4.7) nous founit

$$
\mathscr{F}\left[|x|^{2 p}\left\{\left(\frac{\partial}{\partial x}\right)^{\nu} T_{\lambda}\right\}\right]=\left(-\Delta_{\eta}\right)^{p}\left\{(i \eta)^{\nu} \hat{T}_{\lambda}(\eta)\right\} \in L^{1}\left(g^{n}\right)
$$

pour tout entier $p$ assez grand, où $\Delta_{\eta}$ est l'opérateur de Laplace. D'où on déduit que $|x|^{2 p}\left\{\left(\frac{\partial}{\partial x}\right)^{\nu} T_{\lambda}\right\}$ est une fonction continue de $(x, \lambda)$ dans $\boldsymbol{R}^{n} \times \Lambda$ et analytique en $\lambda$ par le même raisonnement que dans l'assertion $1^{\circ}$. Alors l'identité (4.9) entraîne l'assertion $2^{\circ}$.

Démonstration du Théorème. Remarquons d'abord que les $\lambda_{j}(\eta)$, $j=1, \cdots, l$ sont, grâce aux hypothèses A. 1)-A. 2), des fonctions analytiques en $\eta$ dans $\Xi^{n}-\{0\}$ et positivement homogènes de degré un. De plus on a les relations :

$$
\lambda_{j}(-\eta)=\lambda_{l-j+1}(\eta), \quad j=1, \cdots, l .
$$

En effect on peut les déduire de (4.1) et (4.2). Alors on a en vertu de l'hypothèse $\mathrm{A} .3$ ) les deux cas suivants.

Cas où $l=2 s$ est pair: 
(4. 11) $\quad \lambda_{1}(\eta)>\cdots>\lambda_{s}(\eta)>0>\lambda_{s+1}(\eta)=-\lambda_{s}(-\eta)>\cdots>\lambda_{2 s}(\eta)=-\lambda_{1}(-\eta)$ pour $\eta \neq 0$.

Cas où $l=2 s+1$ est impair :

$$
\begin{aligned}
\lambda_{1}(\eta)>\cdots>\lambda_{s}(\eta)>\lambda_{s+1}(\eta) \equiv 0>\lambda_{s+2}(\eta)=-\lambda_{s}(-\eta) \\
. \quad>\cdots>\lambda_{2 s+1}(\eta)=-\lambda_{1}(-\eta) \text { pour } \eta \neq 0 .
\end{aligned}
$$

Nous introduisons maintenant les matrices

$$
\begin{aligned}
& P_{j}(\eta)= \frac{1}{2 \pi i} \int_{\Gamma_{j}(\eta)}(\lambda I-A(\eta))^{-1} d \lambda \\
&= \frac{1}{\left(m_{j}-1\right) !}\left(\frac{d}{d \lambda}\right)^{m_{j}-1}\left\{\frac{t \operatorname{cof}(\lambda I-A(\eta))}{\left.\frac{\operatorname{dét}(\lambda I-A(\eta))}{\left(\lambda-\lambda_{j}(\eta)\right)^{m_{j}}}\right\}\left.\right|_{\lambda=\lambda_{j}(\eta)},}\right. \\
& j=1, \cdots, l
\end{aligned}
$$

où $\Gamma_{j}(\eta)$ est une courbe fermée de Jordan dans le sens direct qui entoure la racine $\lambda_{j}(\eta)$ mais aucune des autres pour chaque $\eta . P_{j}(\eta)$ est d'après A.2) la matrice définissant la projection qui applique $\boldsymbol{C}^{N}$ sur l'espace propre correspondant à la valeur propre $\lambda_{j}(\eta)$. De plus on a

$$
\begin{gathered}
P_{1}(\eta)+\cdots+P_{l}(\eta)=I, \\
P_{j}(\eta) P_{k}(\eta)=\delta_{j k} P_{j}(\eta), \quad j, k=1, \cdots, l .
\end{gathered}
$$

En utilisant ces $P_{j}(\eta)$, la matrice $A(\eta)$ s'exprime

$$
A(\eta)=\sum_{j=1}^{l} \lambda_{j}(\eta) P_{j}(\eta)
$$

et pour $\lambda$ non réel, la fonction $(A(\eta)-\lambda I)^{-1}$ de la matrice $A(\eta)$ peut être représentée:

$$
(A(\eta)-\lambda I)^{-1}=\sum_{j=1}^{l} \frac{1}{\lambda_{j}(\eta)-\lambda} P_{j}(\eta) .
$$

Soit maintenant $k^{0}$ un nombre réel $\neq 0$ et arbitrairement fixé. Nous allons démontrer le théorème dans le cas: $l=2 s+1$ et le cas où $k^{0}>0$. Les autres cas se démontrent de la même manière en faisant de petites modifications. On fixe une partition finie de l'unité $\left\{\Theta_{p}\right\}$ sur la sphère unité $\Omega$ de $\boldsymbol{\Xi}^{n}$ :

i) $0 \leqslant \Theta_{p}(\omega) \leqslant 1$, 


$$
\begin{aligned}
& \text { ii) } \Theta_{p} \in C_{0}^{\infty}(\Omega) \text {, les supp } \Theta_{p} \text { étant assez petits, } \\
& \text { iii) } \sum_{p} \Theta_{p}(\omega)=1 \text { sur } \Omega \text {, }
\end{aligned}
$$

où $\omega$ désigne la variable parcourant. On pose

$$
\lambda_{p, j}^{\max }=\operatorname{Max}_{\omega \in \operatorname{Supp} \Theta_{p}} \lambda_{j}(\omega) \quad \text { et } \quad \lambda_{p, j}^{\min }=\operatorname{Min}_{\omega \in \operatorname{Supp} \Theta_{p}} \lambda_{j}(\omega),
$$

et choisit un nombre $\delta>0$ de telle manière que les intervalles

$$
[-4 \delta, 4 \delta],\left[\left(k^{0}-4 \delta\right) / \lambda_{p, j}^{\max },\left(k^{0}+4 \delta\right) / \lambda_{p, j}^{\min }\right], \quad j=1, \cdots, s
$$

soient disjoints pour tout $p$ fixé. Puis on prend les fonctions $\phi_{0}(r)$ et $\phi_{p, j}(r, \omega)$ indéfiniment dérivables vérifiant

$$
\phi_{0}(r)=\left\{\begin{array}{lll}
1 & \text { pour } & |r| \leqslant 3 \delta \\
0 & \text { pour } & |r| \geqslant 4 \delta
\end{array} \quad r \in[0, \infty),\right.
$$

(4.21) $\phi_{p, j}(r, \omega)=\left\{\begin{array}{lll}1 & \text { pour } & \left(k^{0}-3 \delta\right) / \lambda_{p, j}^{\max } \leqslant r \leqslant\left(k^{0}+3 \delta\right) / \lambda_{p, j}^{\min } \\ 0 & \text { pour } & r \geqslant\left(k^{0}+4 \delta\right) / \lambda_{p, j}^{\min } \text { ou } \quad r \leqslant\left(k^{0}-4 \delta\right) / \lambda_{p, j}^{\max } .\end{array}\right.$

En utilisant ces fonctions la formule (4.4) se décompose sous la forme :

$$
\begin{aligned}
& (2 \pi)^{n / 2} E(x ; \lambda)=\mathscr{F}^{-1}\left[(A(\eta)-\lambda I)^{-1}\right] \\
& =\mathscr{F}^{-1}\left[\phi_{0}(|\eta|)(A(\eta)-\lambda I)^{-1}\right] \\
& \quad+\sum_{j=1}^{s} \mathscr{F}^{-1}\left[\sum_{p} \Theta_{p}\left(\frac{\eta}{|\eta|}\right) \frac{\left(1-\phi_{0}(|\eta|)\right)\left(1-\phi_{p, j}(|\eta|, \eta /|\eta|)\right)}{\lambda_{j}(\eta)-\lambda} P_{j}(\eta)\right] \\
& \quad+\sum_{j=s+2}^{2 s+1} \mathscr{F}^{-1}\left[\frac{\left(1-\phi_{0}(|\eta|)\right)}{\lambda_{j}(\eta)-\lambda} P_{j}(\eta)\right]-\frac{1}{\lambda} \mathscr{F}^{-1}\left[\left(1-\phi_{0}(|\eta|)\right) P_{s+1}(\eta)\right] \\
& \quad+\sum_{j=1}^{s} \sum_{p} \mathscr{F}^{-1}\left[\Theta_{p}\left(\frac{\eta}{|\eta|}\right) \frac{\phi_{p, j}(|\eta|, \eta /|\eta|)}{\lambda_{j}(\eta)-\lambda} P_{j}(\eta)\right] \\
& =E_{1}(x: \lambda)+E_{2}(x: \lambda)+E_{3}(x ; \lambda)+E_{4}(x ; \lambda)+E_{5}(x ; \lambda) .
\end{aligned}
$$

Envisageons tout d'abord le premier terme $E_{1}(x ; \lambda)=\mathscr{F}^{-1}\left[\phi_{0}(|\eta|)\right.$ $\left.(A(\eta)-\lambda I)^{-1}\right]$. Si $\lambda$ est dans la région $\left\{\lambda ;\left|\operatorname{Re} \lambda-k^{0}\right|<3 \delta\right\}$ la fonction $\phi_{0}(|\eta|)(A(\eta)-\lambda \mathrm{I})^{-1}$ à support compact est indéfiniment dérivable en $\eta$ dans $g^{n}$ et analytique en $\lambda$. On en déduit que pour tout $\nu,(\partial / \partial x)^{\nu} E_{1}(x ; \lambda)$ est une fonction continue de $(x, \lambda)$ dans $\boldsymbol{R}^{n} \times\left\{\lambda ;\left|\operatorname{Re} \lambda-k^{0}\right|<3 \delta\right\}$ et analytique en $\lambda$.

Examinons ensuite le second terme: 


$$
E_{2}(x ; \lambda)=\mathscr{F}^{-1}\left[\sum_{j=1}^{s} \sum_{p} \Theta_{p}(\eta /|\eta|) \frac{\left(1-\phi_{0}(|\eta|)\left(1-\phi_{p, j}(|\eta|, \eta /|\eta|)\right.\right.}{\lambda_{j}(\eta)-\lambda} P_{j}(\eta)\right] .
$$

On écrit, pour simplifier l'écriture, la fonction entre crochet par $Q(\eta ; \lambda)$. Si $\lambda$ est dans $\left\{\lambda ;\left|\operatorname{Re} \lambda-k^{0}\right| \leqslant 3 \delta\right\}, Q(\eta ; \lambda)$ est une fonction indéfiniment différentiable de $\lambda$ et analytique en $\lambda$. De plus elle jouit de la propriété suivante :

Pour tout $\alpha$, on a la majoration

$$
\left|\left(\frac{\partial}{\partial \eta}\right)^{\infty} Q(\eta ; \lambda)\right| \leqslant C_{\alpha}(1+|\eta|)^{-|\alpha|-1} \text { pour } \eta \in \mathbb{E}^{n},
$$

où $C_{a}$ est une constante qui dépend de $\alpha$, mais pas de $\eta$, et pas de $\lambda$ lorsque $\lambda$ décrit un compact du domaine $\left\{\lambda ;\left|\operatorname{Re} \lambda-k^{0}\right| \leqslant 3 \delta\right\}$. Par suite, $E_{2}(x ; \lambda)$ est en vertu du lemme 4.1 une fonction indéfiniment dérivable de $x$ dans $\boldsymbol{R}^{n}-\{0\}$ et pour tout $\nu,(\partial / \partial x)^{\nu} E_{2}(x ; \lambda)$ est continu en $(x, \lambda)$ dans $\left(\boldsymbol{R}^{n}-\{0\}\right) \times\left\{\lambda ;\left|\operatorname{Re} \lambda-k^{0}\right| \leqslant 3 \delta\right\}$ et analytique en $\lambda$. Il en est de même du troisième terme $E_{3}(x ; \lambda)$ $=\mathscr{F}^{-1}\left[\sum_{j=s+l}^{2 s+1} \frac{\left(1-\phi_{0}(|\eta|)\right)}{\lambda_{j}(\eta)-\lambda} P_{j}(\eta)\right]$. Considérons ensuite le quatrième terme $\quad E_{4}(x ; \lambda)=\lambda^{-1} \mathscr{F}^{-1}\left[\left(1-\phi_{0}(|\eta|) P_{s+1}(\eta)\right]\right.$. La fonction entre crochet (désignée par $Q(\eta)$ ) est une fonction indéfiniment dérivable de $\eta$ et on a pour tout $\alpha$

$$
\left|\left(\frac{\partial}{\partial \eta}\right)^{\alpha} Q(\eta)\right| \leqslant C_{\alpha}(1+|\eta|)^{-|\alpha|} \text { pour } \eta \in \Xi^{n} .
$$

Donc $E_{4}(x ; \lambda)$ est une fonction indéfiniment dérivable de $x$ dans $\boldsymbol{R}^{n}-\{0\}$ et analytique en $\lambda$ dans $\boldsymbol{C}-\{0\}$. Envisageons finalement $E_{5}(x ; \lambda)=\mathscr{F}^{-1}\left[\sum_{j=1}^{s} \sum_{p} \Theta_{p}(\eta /|\eta|) \frac{\phi_{p, j}(|\eta|, \eta /|\eta|)}{\lambda_{j}(\eta)-\lambda} P_{j}(\eta)\right]$.

On écrit $\lambda=k \pm i \varepsilon, \varepsilon>0$. Pour $\varepsilon>0 E_{5}(x ; \lambda)$ s'exprime par la forme intégrale :

(4.23) $\quad E_{5}(x ; k \pm i \varepsilon)$

$$
=(2 \pi)^{-n / 2} \sum_{j=1}^{s} \sum_{p} \int_{\mathbb{\Xi}^{n}} e^{i<x, \eta\rangle} \Theta_{p}(\eta /|\eta|) \frac{\phi_{p, j}(|\eta|, \eta /|\eta|)}{\lambda_{j}(\eta)-\lambda} P_{j}(\eta) d \eta .
$$

En passant dans l'intégrale en coordonnées sphériques $(r, \omega)$, il devient 
$(4.23)^{\prime} \quad E_{5}(x ; k \pm i \varepsilon)$

$$
=(2 \pi)^{-n / 2} \sum_{j=1}^{s} \sum_{p} \int_{\Omega}\left\{\int_{0}^{\infty} e^{i<x, \omega>r} \frac{r^{n-1} \phi_{p, j}(r, \omega)}{r-(k \pm i \varepsilon) / \lambda_{j}(\omega)} d r\right\} \frac{\Theta_{p}(\omega)}{\lambda_{j}(\omega)} P_{j}(\omega) d \omega,
$$

où $d \omega$ désigne l'élément de surface de la sphère unité $\Omega$.

Puisque $\phi_{p, j}(r, \omega)=1$ pour $\omega \in \operatorname{Supp} \Theta_{p}$ et pour $r \in\left[\left(k^{0}-3 \delta\right) / \lambda_{p, j}^{\max }\right.$, $\left.\left(k^{0}+3 \delta\right) / \lambda_{p, j}^{\min }\right]$, on peut prolonger les fonctions analytiques d'une variable réel $r$ :

$$
e^{i<x, \omega>r} \frac{r^{n-1} \phi_{p, j}(r, \omega)}{r-(k+i \varepsilon) / \lambda_{j}(\omega)}\left(\operatorname{resp.} e^{i<x, \omega>r} \frac{r^{n-1} \phi_{p, j}(r, \omega)}{r-(k-i \varepsilon) / \lambda_{j}(\omega)}\right) \quad j=1, \cdots, s
$$

en des fonctions analytiques d'une variable complexe $z$ dans le domaine $\left\{z ; \operatorname{Re} z \in\left[\left(k_{0}-3 \delta\right) / \lambda_{p, j}^{\max },\left(k^{0}+3 \delta\right) / \lambda_{p, j}^{\min }\right]\right.$ et $\left.\operatorname{Im} z<-\varepsilon / \lambda_{p, j}^{\max }\right\}$ (resp. $\left\{z ; \operatorname{Re} z \in\left[\left(k^{0}-3 \delta\right) / \lambda_{p, j}^{\max },\left(k^{0}+3 \delta\right) / \lambda_{p, j}^{\min }\right]\right.$ et $\left.\left.\operatorname{Im} z>-\varepsilon / \lambda_{p, j} \max \right\}\right)$. Par conséquent on peut déplacer, en vertu du théorème de Cauchy, le chemin d'intégration $[0, \infty)$ par le suivant.

$\Gamma_{-}=\left[0,\left(k^{0}-2 \delta\right) / \lambda_{p, j}^{\max }\right] \cup\left[\right.$ la demi-circonférence de rayon $R^{0}=2^{-1}$ $\left((k+2 \delta) / \lambda_{p, j}^{\min }-\left(k^{0}-2 \delta\right) \lambda_{p, j}^{\max }\right)$ et de centre $R^{0}+\left(k^{0}-2 \delta\right) / \lambda_{p, j}^{\max }$ dans le

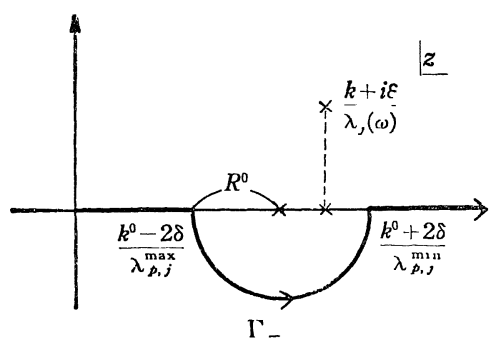

Fig. 1

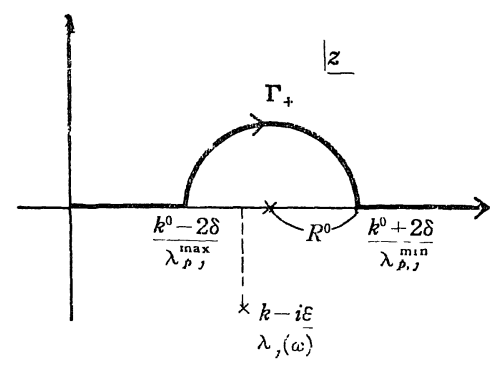

Fig. 2

sens direct, située dans le demi-plan inférieur $\operatorname{Im} z<0] \cup$ $\left[\left(k^{0}+2 \delta\right) / \lambda_{p, j}^{\min }, \infty\right)$ (fig. 1) (resp. $\Gamma_{+}=\left[0,\left(k^{0}-2 \delta\right) / \lambda_{p, j}^{\max }\right] \cup[1 \mathrm{la}$ demicirconférence de centre $R^{0}+\left(k^{0}-2 \delta\right) / \lambda_{p, j}^{\max }$ et de rayon $R_{0}$ dans le sens flèche située dans le demi-plan supérieur $\operatorname{Im} z>0] \cup$ $\left[\left(k^{0}+2 \delta\right) / \lambda_{p, j}^{\min }, \infty\right)$ (fig. 2)). Considérons les fonctions de $(x, \lambda)$ :

$$
\int_{\Omega}\left\{\int_{\Gamma_{\mp}} e^{i<x, \omega>z} \frac{z^{n-1} \phi_{p, j}(z, \omega)}{z-\lambda / \lambda_{j}(\omega)} d z\right\} \frac{(i \omega)^{\nu} \Theta_{p}(\omega)}{\lambda_{j}(\omega)} P_{j}(\omega) d \omega
$$


Elles sont visiblement continues par rapport à $(x, \lambda)$ dans $\boldsymbol{R}^{n} \times\left\{\lambda ;\left|\operatorname{Re} \lambda-k^{0}\right|<\delta\right.$ et $\left.|\operatorname{Im} \lambda|<\varepsilon^{0}\right\} \quad\left(\varepsilon^{0}\right.$ étant un nombre $>0$ et suffisamment petit) et analytiques en $\lambda$. D'où on conclut que la fonction $(\partial / \partial x)^{\nu} E_{5}(x ; \lambda)$ considérée dans le domaine $\boldsymbol{R}^{n} \times\{\lambda ; \mid \operatorname{Re} \lambda$ $-k^{0} \mid<\delta$ et $\left.\operatorname{Im} \lambda>0\right\} \quad$ (resp. $\boldsymbol{R}^{n} \times\left\{\lambda ;\left|\operatorname{Re} \lambda-k^{0}\right|<\delta\right.$ et $\left.\operatorname{Im} \lambda<0\right\}$ se prolonge analytiquement au delà de l'axe réel dans une partie de l'autre demi-plan. De ce que nous avons vu ci-desseus, on tire la conclusion du théorème 4. 1 .

c. q. f. d.

Par le même raisonnement, on a le

Corollaire.-Soient $g$ une fonction dans $C_{0}^{\infty}\left(\boldsymbol{R}^{n}\right)$ et $\nu$ un indice multiple quelconque. Alors les limites

$$
\left(\frac{\partial}{\partial x}\right)^{\nu}(E(x ; k \pm i 0) * g(x))=\lim _{\varepsilon \downarrow 0}\left(\frac{\partial}{\partial x}\right)^{\nu}(E(x ; k \pm i \varepsilon) * g(x))
$$

existent pour $x \in \mathbb{R}^{n}$ et $k \neq 0$ et de plus elles définissent des fonctions continues de $(x, k)$ dans $\boldsymbol{R}^{n} \times\left(\boldsymbol{R}^{1}-\{0\}\right)$.

Considérons maintenant la trace $\left.E(x-y ; \lambda)\right|_{x_{n}=0}=0$ sur l'hyperplan $x_{n}=0, \mathrm{du}$ noyau élémentaire $E(x-y ; \lambda), y$ étant un point quelconque de $\boldsymbol{R}_{+}^{n}$. Nous allons étudier une expression locale de l'image de Fourier de $B E(x-y ; \lambda)_{x_{n}=0}$ par rapport aux variables $x^{\prime}$ $=\left(x_{1}, \cdots, x_{n-1}\right)$, où $B$ est une matrice de type $(l, N)$ à éléments complexes. Pour cela, quant à l'opérateur $A$ (ou $L$ ), revenons encore aux hypothèses originales L.1)-L. 3). Pour $\lambda$ non réel et $y \in \boldsymbol{R}^{n}$, nous avons à partir de la définition (4.4)

$$
E(x-y ; \lambda)=(2 \pi)^{-n / 2 \mathscr{F}} \eta^{-1}\left[e^{-i<y, \eta>}(A(\eta)-\lambda I)^{-1}\right] .
$$

En notant $\tau$ au lieu de $\eta_{n}$ et $\xi=\left(\xi_{1}, \cdots, \xi_{n-1}\right)$ au lieu de $\eta^{\prime}=\left(\eta_{1}, \cdots, \eta_{n-1}\right)$, et en écrivant

$$
(A(\eta)-\lambda I)^{-1}=(\tau I-M(\xi ; \lambda))^{-1} A_{n}^{-1},
$$

on a

$$
\begin{aligned}
& \text { (4.27) } \quad E(x-y ; \lambda) \\
& =(2 \pi)^{-n / 2} \mathscr{F}_{\xi}^{-\xi^{2}}\left[e^{\left.-i<y^{\prime}, \xi\right\rangle}\left\{(2 \pi)^{-1 / 2} \int_{-\infty}^{+\infty} e^{i\left(x_{n}-y_{n}\right)^{\top}}(\tau I-M(\xi ; \lambda))^{-1} A_{n}^{-1} d \tau\right\}\right],
\end{aligned}
$$

où la transformation réciproque $\mathscr{F}_{\bar{\xi}}^{-1}$ de Fourier partielle par rapport 
aux variables $\xi=\left(\xi_{1}, \cdots, \xi_{n-1}\right)$ est effectuée au sens des distributions. En effet il est facile de justifier la formule (4.27). Donc si $y \in \boldsymbol{R}_{+}^{n}$, on a

$$
\begin{aligned}
& (2 \pi)^{n / 2} \mathscr{F}_{x^{\prime}}\left[E(x-y ; \lambda)_{x_{n}=0}\right] \\
& =e^{-i<y^{\prime}, \xi>}(2 \pi)^{-1 / 2} \int_{-\infty}^{+\infty} e^{-i \tau y_{n}}(\tau I-M(\xi ; \lambda))^{-1} A_{n}^{-1} d \tau .
\end{aligned}
$$

Soient maintenant $\xi^{0}$ un point quelconque de $\Xi^{n-1}$ et $k^{0}$ un nombre réel $\neq 0$. Soient $W=W\left(\xi^{0}\right)$ un petit voisinage de $\xi^{0}$ dans $\Xi^{n-1}$ et $\Lambda_{\delta}^{ \pm}$des domaines du plan complexe, définis dans le $\S 2$. (Voir (2.16)' et $\left.(2.16)^{\prime \prime}\right)$. Pour obtenir une expression plus explicite du second membre de (4.28) dans $W \times \Lambda_{\delta}^{+}$(resp. $W \times \Lambda_{\delta}^{-}$), nous plaçons, quant aux racines caractéristiques de $M\left(\xi^{0} ; k^{0}\right)$, dans la situation (**) du $\$ 2$, plus précisément (2.20), qui ne diminue pas la généralité:

" $\tau_{1}^{-}\left(\xi^{0} ; k^{0}+i 0\right)=\tau_{1}^{-}\left(\xi^{0} ; k^{0}-i 0\right)$ est racine double réelle, $\tau_{2}^{-}\left(\xi^{0} ;\right.$ $\left.k^{0} \pm i 0\right), \cdots, \tau_{\bar{p}}^{-}\left(\xi^{0} ; k^{0} \pm i 0\right)$ sont racines simples réelles et $\tau_{p_{+1}}\left(\xi^{0} ; k^{0} \pm i 0\right)$ $, \cdots, \tau_{m}^{-}\left(\xi^{0} ; k^{0} \pm i 0\right)$ racines non réelles". $(\xi, \lambda)$ étant un point quelconque de $W \times \Lambda_{\delta}^{+}$(resp. $\left.W \times \Lambda_{\delta}^{-}\right)$, on a $\operatorname{Im} \tau_{j}^{-}(\xi ; \lambda)<0, j=1, \cdots, m$. On prend une courbe fermée de Jordan $\Gamma=\Gamma(\xi ; \lambda)$ dans le sens direct, située dans le demi-plan inférieur $\boldsymbol{C}^{-}=\{\tau ; \operatorname{Im} \tau<0\}$ et qui entoure les racines $\tau_{j}^{-}(\xi ; \lambda), j=1, \cdots, m$. Alors on a, en se rappelant que $y_{n}>0$,

(4.29) $\int_{-\infty}^{+\infty} e^{-i \tau y_{n}}(\tau I-M(\xi, \lambda))^{-1} A_{n}^{-1} d \tau=-\int_{\Gamma} e^{-i \tau y_{n}}(\tau I-M(\xi ; \lambda))^{-1} A_{n}^{-1} d \tau$.

En effet, soit $S_{R}$ la demi-circonférence de centre 0 et de rayon $R$ parcourue dans le sens direct, et située dans le demi-plan $\operatorname{Im} \tau<0$. Alors on a

$$
\int_{-R}^{R} e^{-i \tau y_{n}}(\tau I-M(\xi ; \lambda))^{-1} A_{n}^{-1} d \tau=-\int_{\Gamma} " d \tau+\int_{S_{R}}, d \tau
$$

pour tout $R$ suffisamment grand. Du fait que $y_{n}>0$ et de l'estimation évidente

$$
\left|(\tau I-M(\xi ; \lambda))^{-1}\right| \leqslant C /|\tau| \quad \text { pour tout }|\tau| \geqslant \tau^{0}
$$

où $C$ et $\tau^{0}$ sont des constantes positives, on tire

$$
\lim _{R \rightarrow \infty} \int_{S_{R}} e^{-i \tau y_{n}}(\tau I-M(\xi ; \lambda))^{-1} A_{n}^{-1} d \tau=0
$$


Par suite on obtient (4.29) en faisant augmenter $R$ indéfiniment dans (4. 30).

Prenons maintenant une courbe fermée de Jordan $\gamma^{-}$dans le sens direct, contenue en entier dans le demi-plan $\operatorname{Im} \tau<0$, et qui entoure les racines $\left\{\tau_{j}^{-}(\xi ; \lambda) ; p+1 \leqslant j \leqslant m, \xi \in W\right.$ et $\lambda \in \bar{\Lambda}_{\delta}^{+}$(resp. $\bar{\Lambda}_{\delta}^{-}$), mais aucune des autres racines $\left\{\tau_{j}^{-}(\xi ; \lambda) ; 1 \leqslant j \leqslant p, \xi \in W\right.$ et $\lambda \in \bar{\Lambda}_{\delta}^{+}$ (resp. $\left.\left.\bar{\Lambda}_{\delta}^{-}\right)\right\}$. Cela est possible en reprenant au besoin $W$ et $\bar{\Lambda}_{\delta}^{ \pm}$plus petits. Alors l'intégrale du second membre de (4.29) s'écrit

$$
\begin{gathered}
-2 \pi i \sum_{j=1}^{p} \underset{\tau=\tau_{j}^{-}(\xi ; \lambda)}{\operatorname{Rés}}\left\{e^{-i \tau y_{n}}(\tau I-M(\xi ; \lambda))^{-1} A_{n}^{-1}\right\} \\
-\int_{\gamma^{-}} e^{-i \tau y_{n}}(\tau I-M(\xi ; \lambda))^{-1} A_{n}^{-1} d \tau .
\end{gathered}
$$

Or on a

$$
\begin{aligned}
& \underset{\substack{\tau \text { Rés } \\
\tau=\tau_{j}-(\xi ; \lambda)}}{\left\{e^{\left.-i \tau y_{n}(\tau I-M(\xi ; \lambda))^{-1} A_{n}^{-1}\right\}}\right.} \\
& =\left.e^{-i \tau_{j}-(\xi ; \lambda) y_{n}} \frac{{ }^{t}}{\frac{d}{d \tau}\left\{\operatorname{cof}(\tau I-M(\xi ; \lambda)) A_{n}^{-1}\right.}\right|_{\tau=\tau_{j}-(\xi ; \lambda)}
\end{aligned}
$$

Rappelons ici les relations :

$$
\begin{aligned}
& { }^{t} \operatorname{cof}(\tau I-M(\xi ; \lambda)) A_{n}^{-1}=\operatorname{dét}\left(A_{n}^{-1}\right)^{t} \operatorname{cof}(A(\xi, \tau)-\lambda I) \text { et } \\
& (A(\xi, \tau)-\lambda I){ }^{t} \operatorname{cof}(A(\xi, \tau)-\lambda I)=\operatorname{dét}\left(A_{n}\right) \operatorname{dét}(\tau I-M(\xi ; \lambda)) I .
\end{aligned}
$$

En désignant par $\boldsymbol{q}_{\rho}(\xi, \tau, \lambda)$ la $\rho$-ième colonne de la matrice ${ }^{t}$ cof $(A(\xi, \tau)-\lambda I), \quad \boldsymbol{q}_{\rho}\left(\xi, \tau_{j}^{-}(\xi, \lambda), \lambda\right)(1 \leqslant j \leqslant p)$ est un vecteur propre pour la valeur propre $\tau_{j}^{-}(\xi ; \lambda)$ de $M(\xi ; \lambda)$. Donc il existe des fonctions $\alpha_{\rho_{j}}$ telles que

$$
\begin{aligned}
-\operatorname{dét}\left(A_{n}^{-1}\right) \boldsymbol{q}_{\rho}\left(\xi, \tau_{j}^{-}(\xi ; \lambda), \lambda\right)=\alpha_{\rho_{j}}(\xi ; \lambda) \boldsymbol{h}_{j}^{-}(\xi ; \lambda), & \\
& \rho=1, \cdots, 2 m, \quad j=1, \cdots, p .
\end{aligned}
$$

Ici les $\boldsymbol{h}_{j}^{-}(\xi ; \lambda), j=1, \cdots, p$ sont les vecteurs correspondant aux $\tau_{j}^{-}(\xi ; \lambda), j=1, \cdots, p$, respectivement, définis dans le $\S 2$.

On déduit de (4.28), (4.29), (4.31), (4.32) et (4.33) l'expression suivante dans $W \times \Lambda_{\delta}^{ \pm}$: 
(4.34) $\mathscr{F}_{x^{\prime}}\left[E(x-y ; \lambda) \underset{\lambda_{n}=0}{\mid}\right]$

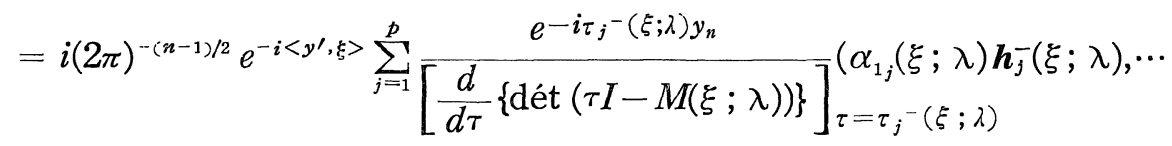

$$
\begin{aligned}
& \left.\cdots, \alpha_{2 m, j}(\xi ; \lambda) \boldsymbol{h}_{j}^{-}(\xi ; \lambda)\right) \\
& -(2 \pi)^{-(n+1) / 2} e^{\left.-i<y^{\prime}, \xi\right\rangle} \int_{\gamma^{-}} e^{-i \tau y_{n}}(\tau I-M(\xi ; \lambda))^{-1} A_{n}^{-1} d \tau .
\end{aligned}
$$

Par conséquent on a, avec $\boldsymbol{h}_{j}^{-}=\boldsymbol{h}_{j}^{-}(\xi ; \lambda)$,

$$
\begin{aligned}
& \text { (4. 35) } \mathscr{F}_{x^{\prime}}\left[B E(x-y ; \lambda){ }_{x_{n}=0}^{\mid}\right] \\
&= i(2 \pi)^{-(n-1) / 2} e^{-i<y \prime, \xi>} \sum_{j=1}^{p} \frac{e^{-i \tau_{j}-(\xi ; \lambda) y_{n}}}{\left[\frac{d}{d \tau}\{\operatorname{dét}(\tau I-M(\xi ; \lambda))\}\right]_{\tau=\tau_{j}^{-}(\xi ; \lambda)}} \\
& \times\left(\begin{array}{c}
\alpha_{1 j}(\xi ; \lambda)\left\langle\boldsymbol{b}_{1}, \boldsymbol{h}_{j}^{-}\right\rangle, \cdots, \alpha_{2 m, j}(\xi ; \lambda)\left\langle\boldsymbol{b}_{1}, \boldsymbol{h}_{j}^{-}\right\rangle \\
\vdots \\
\alpha_{1 j}(\xi ; \lambda)\left\langle\boldsymbol{b}_{m}, \boldsymbol{h}_{j}^{-}\right\rangle, \cdots, \alpha_{2 m, j}(\xi ; \lambda)\left\langle\boldsymbol{b}_{m}, \boldsymbol{h}_{j}^{-}\right\rangle
\end{array}\right) \\
&-(2 \pi)^{-(n+1) / 2} e^{-\left\langle y^{\prime}, \xi\right\rangle} \int_{\gamma^{-}} e^{-i \tau y_{n}} B(\tau I-M(\xi ; \lambda))^{-1} A_{n}^{-1} d \tau .
\end{aligned}
$$

§5. Matrice-noyau de Green du Problème aux Limites $\{A-\lambda I, B\}$

Dans ce pragraphe, nous revenons de nouveau le problème aux limites (1. 19) et (1.20):

$$
\begin{gathered}
(A-\lambda I) U(x ; \lambda)=g(x), \quad x \in \boldsymbol{R}_{+}^{n} \\
B U(x ; \lambda)_{x_{n}=0}=0
\end{gathered}
$$

sous les hypothèses L. 1)-L. 3) et B. 1)-B. 2) sur $L$ et sur $B$ respectivement. La matrice-noyau de Green $G(x, y ; \lambda)$ du problème (5.1)(5.2) avec $\lambda$ non réel, est par définition la solution de l'équation

$$
(A-\lambda I) G(x, y ; \lambda)=\delta(x-y) I \quad \text { pour } x, y \in \boldsymbol{R}_{+}^{n},
$$

vérifiant la condition aux limites

$$
B G(x, y ; \lambda) \underset{x_{n}=0}{\mid}=0,
$$

où $\delta(x-y)$ est la distribution de Dirac au point $y$ de $\boldsymbol{R}_{+}^{n}$. Nous allons construire le noyau. Comme nous l'avons déjà mentionné dans le $\S 1$, nous posons, conformément à un procédé habituel, le 
noyau de Green cherché sous la forme

$$
G(x, y ; \lambda)=E(x-y ; \lambda)-E_{c}(x, y ; \lambda),
$$

où $E(x ; \lambda)$ est la solution élémentaire de l'opérateur $A-\lambda I$, définie par la formule (4.4) dans le $\S 4$. Alors $E_{c}(x, y ; \lambda)$ que l'on appelle noyau compensateur, s'obtient comme solution du problème aux limites avec $\lambda$ non réel

$$
\begin{gathered}
(A-\lambda I) E_{c}(x, y ; \lambda)=0 \quad \text { pour } x, y \in \mathbb{R}_{+}^{n} \\
B E_{c}(x, y ; \lambda)_{x_{n}=0}=B E\left((x-y ; \lambda)_{x_{n}=0} .\right.
\end{gathered}
$$

En effectuant formellement la transformation de Fourier partielle par rapport aux variables $x^{\prime}=\left(x_{1}, \cdots, x_{n-1}\right)$, nous avons le système d'equations différentiells ordinaires de la variable $x_{n}$, dépendant des paramètres $\xi$ et $\lambda$

$$
\left(\frac{1}{i} \frac{d}{d x_{n}}-M(\xi ; \lambda)\right) \widetilde{E}_{c}\left(\xi, x_{n}, y ; \lambda\right)=0, \quad x_{n}>0
$$

et la condition

$$
B \widetilde{E}_{c}(\xi, 0, y ; \lambda)=\mathscr{F}_{x}\left[B E(x-y ; \lambda)_{x_{n}=0}\right],
$$

où $\widetilde{E}_{c}$ désigne la transformée de Fourier partielle de $E_{c}$ par rapport à $x^{\prime}$. Sous les hypothèses : la symétrie des $A_{j}, j=1, \cdots, n$ et B. 1 ), il existe, grâce aux lemmes 3.1 et 3.3 , une solution unique du problème (5.8)-(5.9), appartient à $L^{2}\left(\boldsymbol{R}_{+}^{1}\right)$, pour tout $\xi \in \boldsymbol{G}^{n-1}$ et tout $\lambda$ non réel. En utilisant la solution $\widetilde{E}_{c}\left(\xi, x_{r}, y ; \lambda\right)$ dépendant des paramètres $\xi$ et $\lambda$, le noyau compensateur cherché est donné par son image réciproque de Fourier par rapport à $\xi=\left(\xi_{1}, \cdots, \xi_{n-1}\right)$ :

$$
E_{c}(x, y ; \lambda)=\mathscr{F}_{\xi}^{-1}\left[\widetilde{E}_{c}\left(\xi, x_{z}, y ; \lambda\right)\right] \text {. }
$$

Notre tâche est maintenant d'obtenir une expression plus explicite de $\widetilde{E}_{c}\left(\xi, x_{n}, y ; \lambda\right)$. Si on désigne par $U_{\rho}\left(\xi, x_{n}, y ; \lambda\right)$ la $\rho$-ième colonne de la matrice $\widetilde{E}_{c}\left(\xi, x_{n}, y ; \lambda\right)$ à $m$ lignes et $N(=2 m)$ colonnes, elle est donnée par la formule:

(5. 11) $U_{\rho}\left(\xi, x_{n}, y ; \lambda\right)=\frac{1}{2 \pi i} \int_{\Gamma^{+}} e^{i \tau x_{n}}(\tau I-M(\xi ; \lambda))^{-1} U_{\rho}(\xi, 0, y ; \lambda) d \tau$, $\Gamma^{+}=\Gamma^{+}(\xi ; \lambda)$ est ici une courbe fermée de Jordan, dans le sens 
direct, contenue tout entière dans le demi-plan $\{\tau ; \operatorname{Im} \tau>0\}$ et qui entoure les racines $\tau_{j}^{+}(\xi ; \lambda), j=1, \cdots, m$. Il existe une telle courbe puisque $\operatorname{Im} \tau_{j}^{+}(\xi ; \lambda)>0, j=1, \cdots, m$ pour $\lambda$ non réel.

Pour trouver, dans l'espace $E^{+}(\xi ; \lambda), U_{\rho}\left(\xi, x_{n}, y ; \lambda\right)$ vérifiant (5. 9), nous posons

$$
U_{\rho}(\xi, 0, y ; \lambda)=\sum_{j=1}^{m} C_{\rho_{j}} h_{j}^{+}(\xi ; \lambda)
$$

et déterminons les coefficients $C_{\rho j}=C_{\rho j}(\xi, y ; \lambda), j=1, \cdots, m$. Comme ces coefficients doivent vérifier par (5.9) le système d'équations linéaires :

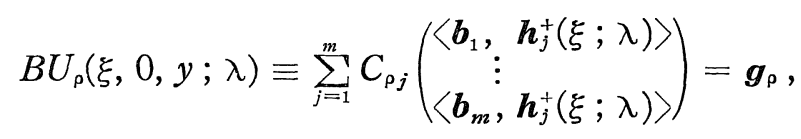

on a en vertu du lemme 3.3

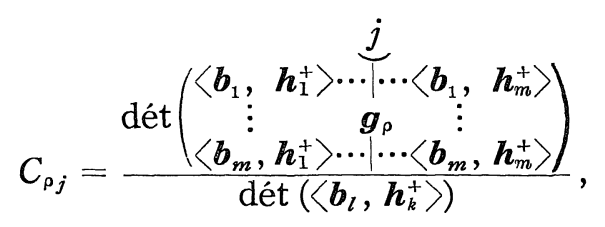

où $\boldsymbol{g}_{\mathrm{\rho}}$ désigne la $\rho$-ième colonne de la matrice $\mathscr{I}_{x^{\prime}}\left[B E(x-y ; \lambda)_{x_{n}=0} \mid\right]$. D'après (4.35) on trouve

$$
\begin{aligned}
& C_{\rho j}=i(2 \pi)^{-(n-1) / 2} \sum_{l=1}^{p} \frac{\alpha_{\rho l}(\xi ; \lambda) e^{-i\left\langle y^{\prime}, \xi\right\rangle-i \tau_{l}-(\xi ; \lambda) y_{n}}}{\Delta(\xi ; \lambda)\left[\frac{d}{d \tau}\{(\tau I-M(\xi ; \lambda))\}\right]_{\tau=\tau_{l}-(\xi ; \lambda)}}
\end{aligned}
$$

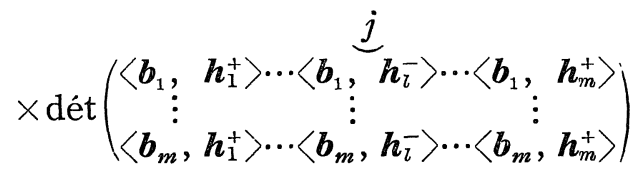

$$
\begin{aligned}
& -\frac{(2 \pi)^{-(n+1) / 2} e^{\left.-i<y^{\prime}, \xi\right\rangle} \operatorname{dét}\left(A_{n}^{-1}\right)}{\Delta(\xi, \lambda)} \int_{\gamma^{-}} \frac{e^{-i \tau y_{n}}}{\operatorname{dét}(\tau I-M(\xi ; \lambda))} \\
& \times \operatorname{dét}\left(\begin{array}{cc}
\left\langle\boldsymbol{b}_{1},\right. & \left.\boldsymbol{h}_{1}^{+}\right\rangle \cdots\left\langle\boldsymbol{b}_{1}, \quad \boldsymbol{q}_{\rho}(\xi, \tau, \lambda)\right\rangle \cdots\left\langle\boldsymbol{b}_{1},\right. \\
\vdots & \left.\boldsymbol{h}_{m}^{+}\right\rangle \\
\left\langle\boldsymbol{b}_{m},\right. & \left.\left.\boldsymbol{h}_{1}^{+}\right\rangle \cdots<\boldsymbol{b}_{m}, \boldsymbol{q}_{\rho}(\xi, \tau, \lambda)\right\rangle \cdots\left\langle\boldsymbol{b}_{m}, \boldsymbol{h}_{m}^{+}\right\rangle
\end{array}\right) d \tau
\end{aligned}
$$

où $\boldsymbol{q}_{\rho}(\xi, \tau, \lambda)$ désigne la $\rho$-ième colonne de la matrice ${ }^{t} \operatorname{cof}(A(\xi, \tau)-\lambda I)$.

Ainsi nous tirons de (5.11) et (5.12) l'expression: 


$$
\begin{aligned}
& U_{\rho}\left(\xi, x_{n}, y ; \lambda\right)=\sum_{j=1}^{p} C_{\rho_{j}} e^{i \tau_{j}+(\xi ; \lambda) x_{n}} \boldsymbol{h}_{j}^{+}(\xi ; \lambda) \\
& \quad+\frac{1}{2 \pi i} \sum_{j=p+1}^{m} C_{\rho_{j}}\left(\int_{\gamma^{+}} e^{i \tau x_{n}}(\tau I-M(\xi ; \lambda))^{-1} d \tau\right) \boldsymbol{h}_{j}^{+}(\xi ; \lambda)
\end{aligned}
$$

Ici les $C_{\rho j}$ se définissent par (5.15) et $\gamma^{+}$est une courbe fermée de Jordan, contenue en entier dans le demi-plan $\boldsymbol{C}^{+}=\{\tau ; \operatorname{Im} \tau>0\}$ et qui entoure les racines $\left\{\tau_{j}(\xi ; \lambda) ; p+1 \leqslant j \leqslant m, \xi \in W=W\left(\xi^{0}\right)\right.$ et $\lambda \in \bar{\Lambda}_{\delta}^{+}=\bar{\Lambda}_{\delta}^{+}\left(k^{0}\right)$ (resp. $\left.\left(\bar{\Lambda}_{\delta}^{-}\right)\right\}$, mais aucune des autres racines $\left\{\tau_{j}^{+}(\xi ; \lambda)\right.$, $1 \leqslant j \leqslant p, \xi \in W$ et $\left.\lambda \in \bar{\Lambda}_{\delta}^{+}\left(\operatorname{resp} . \bar{\Lambda}_{j}^{-}\right)\right\}$. Cette expression est locale par rapport aux $\xi$ et $\lambda$, à savoir qu'elle est valable dans $W \times \Lambda_{\delta}^{+}$ (resp. $W \times \Lambda_{\delta}^{-}$).

Nous allons maintenant envisager une expression de $U_{\rho}\left(\xi, x_{n}, y ; \lambda\right)$ qui est valable pour tout $\xi$ tel que $|\xi| \geqslant R$ et pour $\lambda \in \Lambda_{\delta}\left(k^{0}\right), k^{0}$ étant un nombre réel $\neq 0$ et $R$ un nombre positif suffisamment grand. En écrivant $\tau^{*}=\tau / \sqrt{|\xi|^{2}+|\tau|^{2}}$, considérons l'équation en $\tau^{*}$;

$$
\operatorname{dét}\left(\tau^{*} I-M\left(\xi / \sqrt{|\xi|^{2}+|\lambda|^{2}}, \lambda / \sqrt{|\xi|^{2}+|\lambda|^{2}}\right)\right)=0 .
$$

$\mathrm{Si}$ on prend un nombre $R>0$ assez grand, les nombres $\left\{\left|\lambda^{*}\right|=|\lambda| / \sqrt{|\xi|^{2}+|\lambda|^{2}} ;|\xi| \geqslant R\right.$ et $\left.\lambda \in \Lambda_{\delta}\right\}$ sont suffisamment petits. Par suites toutes les racines de l'équation (5.17) pour de tels $\xi$ et $\lambda$ sont non réelles en vertu de l'ellipticité de l'opérateur $A$ et de plus la distance de l'ensemble des racines $\left\{\tau^{*}(\xi ; \lambda) ; \operatorname{Im} \tau^{*}(\xi ; \lambda)>0,|\xi| \geqslant R\right.$ et $\left.\lambda \in \Lambda_{\delta}\right\}$ à l'axe réel est positive. Il en est de même de l'ensemble des racines $\tau^{*}(\xi ; \lambda)$ à partie imaginaire négative. On prend alors une courbe fermée de Jordan $\gamma_{*}^{+}\left(\right.$resp. $\left.\gamma_{*}\right)$ dans le demi-plan $\{\tau ; \operatorname{Im} \tau>0\}$ (resp. $(\{\tau ; \operatorname{Im} \tau<0\})$ qui entoure les racines $\left\{\tau^{*}(\xi ; \lambda) ; \operatorname{Im} \tau^{*}(\xi ; \lambda)>0\right.$, $|\xi| \geqslant R$ et $\left.\lambda \in \bar{\Lambda}_{\delta}\right\}$ (resp. $\left\{\tau^{*}(\xi ; \lambda) ; \operatorname{Im} \tau^{*}(\xi ; \lambda)<0,|\xi| \geqslant R\right.$ et $\left.\lambda \in \bar{\Lambda}_{\delta}\right\}$ ).

Soit $\xi^{0}$ un point quelconque tel que $\left|\xi^{0}\right|>R$ et soit $\left\{\boldsymbol{h}_{j}^{0}\right\}_{j=1, \cdots m}$ une base de $E^{+}\left(\xi^{0} ; k^{0}\right)$. On pose

$$
\begin{aligned}
\boldsymbol{h}_{j}^{+}(\xi ; \lambda)= & \frac{1}{2 \pi i} \int_{r_{*}^{+}}\left(\tau^{*} I-M\left(\xi / \sqrt{|\xi|^{2}+|\lambda|^{2}},\right.\right. \\
& \left.\left.\lambda / \sqrt{|\xi|^{2}+|\lambda|^{2}}\right)\right)^{-1} \boldsymbol{h}_{j}^{0} d \tau^{*}, \quad j=1, \cdots, m .
\end{aligned}
$$

Les $\boldsymbol{h}_{j}^{+}(\xi ; \lambda) j=1, \cdots, m$ se définissent dans $\{\xi ;|\xi| \geqslant R\} \times \Lambda_{\delta}$ et forment une base de $E^{+}(\xi ; \lambda)$ pour chaque $(\xi, \lambda)$. On peut aussi 
définir de la même manière un système $\left\{\boldsymbol{h}_{\boldsymbol{j}}^{-}(\xi ; \lambda)\right\}_{j=1, \cdots, m}$ de vecteurs définis dans $\{\xi ;|\xi| \geqslant R\} \times \Lambda_{\delta}$ et qui forment une base de $E^{-}(\xi ; \lambda)$ pour chaque $(\xi, \lambda)$. En utilisant les $\boldsymbol{h}_{j}^{+}(\xi ; \lambda), j=1, \cdots, m$, on définit le déterminant de Lopatinski $\Delta(\xi ; \lambda)$ pour $\xi(|\xi| \geqslant R)$ et $\lambda \in \Lambda_{\delta}$ par la formule (2.26), c'est-à-dire, par dét $\left(\left\langle\boldsymbol{b}_{i}, \boldsymbol{h}_{j}^{+}(\xi ; \lambda)\right\rangle\right)$. Alors on a, en vertu des lemmes 3.3 et $3.5, \Delta(\xi ; \lambda) \neq 0$ pour $\xi(|\xi| \geqslant R)$ et $\lambda \in \Lambda_{\delta}$. Ceci préparé, passons au problème de l'expression de $\widetilde{E}_{c}\left(\xi, x_{n}, y ; \lambda\right)$ pour $\xi(|\xi| \geqslant R)$ et $\lambda \in \Lambda_{\delta}$. D'abord l'expression de $\mathscr{F}_{x^{\prime}}[B E(x-y ; \lambda) \mid]$ correspond à la formule (4.35), prend la forme :

$$
\begin{aligned}
& \mathscr{F}_{x^{\prime}}\left[\left.B E(x-y ; \lambda)\right|_{x_{n}=0}\right] \\
= & -(2 \pi)^{-(n+1) / 2} e^{-i<y \prime, \xi>} \int_{\gamma_{*}^{-}} e^{-i y_{n} \sqrt{|\xi|^{2}+|\lambda|^{2}} \tau^{*}} \\
& \times B\left(\tau^{*} I-M\left(\xi / \sqrt{|\xi|^{2}+|\lambda|^{2}}, \lambda / \sqrt{|\xi|^{2}+|\lambda|^{2}}\right)\right) A_{n}^{-1} d \tau^{*},
\end{aligned}
$$

pour $\xi(|\xi| \geqslant R)$ et $\lambda \in \Lambda_{\delta}$. Donc les coefficients $C_{\rho_{j}}=C_{\rho_{j}}(\xi, y ; \lambda)$ de (5.12) sont données pour $\xi(|\xi| \geqslant R)$ et $\lambda \in \Lambda_{\delta}$ par la formule suivante.

$$
\begin{aligned}
& C_{\rho_{j}}=-(2 \pi)^{-(n+1) / 2} \frac{e^{-i<y^{\prime}, \xi>} \operatorname{dét}\left(A_{n}^{-1}\right)}{\Delta(\xi ; \lambda)} \\
& \times \int_{\gamma^{-}} \frac{e^{-i \sqrt{|\xi|^{2}+|\lambda|^{2}} y_{n} \tau^{*}}}{\operatorname{dét~}\left(\tau^{*} I-M\left(\xi / \sqrt{|\xi|^{2}+|\lambda|^{2}}, \lambda / \sqrt{\left.|\xi|^{2}+|\lambda|^{2}\right)}\right)\right.} \\
& \times \operatorname{dét}\left(\begin{array}{c}
\left\langle\boldsymbol{b}_{1}, \quad \boldsymbol{h}_{1}^{+}(\xi ; \lambda)\right\rangle \cdots\left\langle\boldsymbol{b}_{1}, \boldsymbol{q}_{\rho}\left(\xi / \sqrt{|\xi|^{2}+|\lambda|^{2}},\right.\right. \\
\vdots \\
\left\langle\boldsymbol{b}_{m}, \boldsymbol{h}_{1}^{+}(\xi ; \lambda)\right\rangle \cdots\left\langle\boldsymbol{b}_{m}, \boldsymbol{q}_{\rho}\left(\xi / \sqrt{|\xi|^{2}+|\lambda|^{2},}\right.\right. \\
\left.\left.\tau^{*}, \lambda / \sqrt{|\xi|^{2}+|\lambda|^{2}}\right)\right\rangle \cdots\left\langle\boldsymbol{b}_{1}, \boldsymbol{h}_{m}^{+}(\xi ; \lambda)\right\rangle \\
\vdots \\
\left.\left.\tau^{*}, \lambda / \sqrt{|\xi|^{2}+|\lambda|^{2}}\right)\right\rangle \cdots\left\langle\boldsymbol{b}_{m}, \boldsymbol{h}_{m}^{+}(\xi ; \lambda)\right\rangle
\end{array}\right) d \tau^{*} .
\end{aligned}
$$

On a alors l'expression suivante de $U_{\rho}\left(\xi, x_{n}, y ; \lambda\right)$ pour $\xi(|\xi| \geqslant R)$ et $\lambda \in \Lambda_{\delta}$.

$$
\begin{aligned}
& U_{\rho}\left(\xi, x_{n}, y ; \lambda\right)=\frac{1}{2 \pi i} \sum_{j=1}^{m} C_{\rho j}(\xi, y ; \lambda) \\
& \times\left(\int _ { r _ { * } ^ { + } } e ^ { i x _ { n } \sqrt { | \xi | ^ { 2 } + | \lambda | ^ { 2 } } \tau ^ { * } } \left(\tau^{*} I-M\left(\xi / \sqrt{|\xi|^{2}+|\lambda|^{2}}\right.\right.\right. \\
& \left.\left.\left.\lambda / \sqrt{|\xi|^{2}+|\lambda|^{2}}\right)\right)^{-1} d \tau^{*}\right) \boldsymbol{h}_{j}^{+}(\xi ; \lambda),
\end{aligned}
$$


les coefficients $C_{\rho_{j}}$ étant donnés par (5.20).

A l'aide des expressions (5.16) avec (5.15) et (5.21) avec (5.20), on peut démontrer le

Théorème 5.1. On pose $\lambda=k \pm i \varepsilon, k$ réel $\neq 0$ et $\varepsilon>0$. Pour tout indice multiple $\nu$ et pour $x, y \in \boldsymbol{R}_{+}^{n}$, les limites suivantes existent :

$$
E_{c}^{(\nu)}(x, y ; k \pm i 0)=\lim _{\varepsilon \downarrow 0}\left(\frac{\partial}{\partial x}\right)^{\nu} E_{c}(x, y ; k \pm i \varepsilon)
$$

et $E_{c}^{(\nu)}(x, y ; k \pm i 0)$ sont continues par rapport $\dot{a}(x, y, k)$ dans la région $\left\{(x, y, k) ; x, y \in \mathbb{R}_{+}^{n}\right.$ et $\left.k \neq 0\right\}$.

Démonstration. Soient $k^{0}$ un nombre réel quelconque $\neq 0$ et $\delta$ un nombre positif suffisamment petit. Il suffit alors de démontrer le théorème pour $k \in\left(k^{0}-\delta, k^{0}+\delta\right)$. On choisit un nombre $R>0$ assez grand de la manière que les formules (5.20) et (5.21) soient valables pour $\xi(|\xi| \geqslant R)$ et $\lambda \in \Lambda_{\delta}$. Prenons une fonction $\varphi \in C_{0}^{\infty}\left(\Xi^{n-1}\right)$, égale à 1 pour $|\xi| \leqslant R+1$ et au zéro pour $|\xi| \geqslant R+2$ et considérons la fonction $(1-\varphi(\xi)) U_{\rho}\left(\xi, x_{n}, y ; \lambda\right)$. Elle est une fonction indéfiniment différentiable de $(x, y, \xi)$ dans $\boldsymbol{R}_{+}^{n} \times \boldsymbol{R}_{+}^{n} \times \mathbb{E}^{n-1}$ et analytique en $\lambda$ dans $\Lambda_{\delta}$, et on déduit de (5.21) avec (5.20) la majoration

$$
\left|(1-\varphi(\xi)) U_{\rho}\left(\xi, x_{n}, y ; \lambda\right)\right| \leqslant \text { Const } e^{-d\left(x_{n}+y_{n}\right) \sqrt{|\xi|^{2}+|\lambda|^{2}}},
$$

$d$ étant un nombre positif convenable. En utilisant cette majoration, on tire de la formule (5.21) avec (5.20) que $\mathscr{F}_{\xi}^{-1}[(1-\varphi(\xi))$ $\left.\times U_{\rho}\left(\xi, x_{n}, y ; \lambda\right)\right]$ est une fonction indéfiniment dérivable de $(x, y)$ dans $\boldsymbol{R}_{+}^{n} \times \boldsymbol{R}_{+}^{n}$ et analytique en $\lambda$ dans $\Lambda_{\delta}$. Envisageons ensuite $\mathscr{F}_{\xi}^{-1}\left[\varphi(\xi) U_{\rho}\left(\xi, x_{n}, y ; k \pm i \varepsilon\right)\right]$. Comme d'habitude nous décrivons seulement le cas où $\lambda=k+i \varepsilon$. La fonction $\phi(\xi) U_{\rho}\left(\xi, x_{n}, y ; k+i \varepsilon\right)$ est indéfiniment dérivable en $\xi$ quand $\varepsilon>0$ et son support (par rapport à $\xi$ ) est compact. Donc, sa transformée de Fourier inverse par rapport à $\xi$ se définit par la forme intégrale. Nous allons montrer que dans l'intégrale on peut faire le passage à la limite sous le signe d'intégration quand on fait tendre $\varepsilon>0$ vers zéro. En utilisant une partition finie et indéfiniment différentiable de l'unité sur $\{\xi ;|\xi|$ $\leqslant R+3\}$, on est ramené au cas où le support de $\varphi(\xi)$ est contenu dans un petit voisinage $W=W\left(\xi^{0}\right)$ d'un point $\xi^{0}\left(\left|\xi^{0}\right| \leqslant R+2\right)$. Con- 
sidérons d'abord le cas où la matrice $M\left(\xi^{0} ; k^{0}\right)$ n'admet pas de racines caractéristiques doubles réelles. Dans ce cas on a, en vertu des lemmes 3.3 et $3.5, \Delta(\xi ; \lambda) \neq 0$ pour $\xi \in W$ et $\lambda \in \bar{\Lambda}_{\delta}^{+}$en reprenant au besoin $W$ et $\delta$ plus petits. Alors l'expression (5.16) avec (5.15) nous montre que les fonctions de $\xi$, dépendant des paramètres $x, y$ et $\lambda=k+i \varepsilon$ :

$$
e^{i<x^{\prime}, \xi>} \varphi(\xi) U_{\rho}\left(\xi, x_{n}, y ; k+i \varepsilon\right)
$$

sont intégrables uniformément par rapport aux $x, y$ et $\lambda$ lorsque $(x, y)$ décrit un compact de $\boldsymbol{R}_{+}^{n} \times \boldsymbol{R}_{+}^{n}$ et que $\lambda=k+i \varepsilon$ décrit $\bar{\Lambda}_{\delta}^{+}$. Donc on voit, en passant la limite sous le signe d'intégration, que

$$
\lim _{\varepsilon \downarrow 0} \int e^{i<x^{\prime}, \xi>} \varphi(\xi) U_{\rho}\left(\xi, x_{n}, y ; k+i \varepsilon\right) d \xi
$$

existe et elle est une fonction indéfiniment différentiable de $(x, y)$ dans $\boldsymbol{R}_{+}^{n} \times \boldsymbol{R}_{+}^{n}$ et continue en $k$ dans $\left(k^{0}-\delta, k^{0}+\delta\right)$.

Passons maintenant au cas où $M\left(\xi^{0} ; k^{0}\right)$ admet un couple de racines caractéristiques doubles réelles. Dans ce cas le déterminant de Lopatinski $\Delta(\xi ; k+i 0)$ peut s'annuler au point $(\xi, k)=\left(\xi^{0}, k^{0}\right)$. En vertu de l'expression (5.16) avec (5.15) on voit que l'intégrabilité des fonctions (5.24) de $\xi$ pour $\lambda=k+i \varepsilon \in \bar{\Lambda}_{\delta}^{+}$se ramène à celle des $C_{\rho j}=C_{\rho j}(\xi, y ; k+i \varepsilon)$. Dans l'expression (5.15) il y a deux facteurs :

$$
\Delta(\xi ; k+i \varepsilon) \text { et }\left[\frac{d}{d \tau}\{\operatorname{dét}(\tau I-M(\xi ; \lambda))\}\right]_{\tau=\tau_{\iota^{-}}(\xi ; \lambda)},
$$

ayant la possibilité de détruire l'intégrabilité $\operatorname{des} C_{\rho_{j}}(\xi, y ; k+i \varepsilon)$ lorsque $\varepsilon$ tend vers zéro. Le deuxième terme dans le second membre de la formule (5.15) ne contient qu'un tel facteur $\Delta(\xi ; k+i \varepsilon)$. Compte tenu de l'expression (3.16) de $\Delta(\xi ; \lambda)$ dans $W \times \bar{\Lambda}_{\delta}^{+}$, on trouve que le deuxième terme est intégrable uniformément par rapport aux $x, y$ et $\lambda$ dans (un compact de $\boldsymbol{R}_{+}^{n} \times \boldsymbol{R}_{+}^{n}$ ) $\times \bar{\Lambda}_{\delta}^{+}$. Examinons ensuite l'intégrabilité uniforme du premier terme du second membre de (5.15). D'après la convention faite dans le $\$ 2$, la racine-fonction à partie imaginaire non négative (resp. non positive) correspondant à un couple de racines caractéristiques doubles réelles de $M\left(\xi^{0} ; k^{0}\right)$, c'est $\tau_{1}^{+}(\xi ; k+i \varepsilon)$ (resp. $\tau_{1}^{-}(\xi ; k+i \varepsilon)$ ). Et les $\tau_{j}^{ \pm}(\xi ; \lambda), j=2, \cdots, p$ sont racines simples réelles pour $(\xi, \lambda) \in W \times \bar{\Lambda}_{\delta}^{+}$. On a donc 


$$
\left[\frac{d}{d \tau}\{\text { dét }(\tau I-M(\xi ; \lambda))\}\right]_{\tau=\tau_{l}-(\xi ; \lambda)} \neq 0 \quad \text { pour } l=2, \cdots, p .
$$

Par suite l'intégrabilité uniforme du terme $\sum_{l=2}^{p}$, dans l'expression (5.15) est garantie par la même raison qu'au cas précédent. Envisageons finalement le terme où $l=1$. Pour $(\xi, k)$ tel que $M(\xi ; k)$ admette des racines caractéristiques doubles réelles, on a

$$
\left[\frac{d}{d \tau}\{\text { dét }(\tau I-M(\xi ; k))\}\right]_{\tau=\tau_{l}-(\xi ; k)}=0
$$

et $\Delta(\xi ; k+i 0)$ peut s'annuler. Mais grâce à la relation (2.25): $\boldsymbol{h}_{1}^{+}(\xi ; k+i 0)=\boldsymbol{h}_{1}^{-}(\xi ; k+i 0)$, on a pour un tel $(\xi, k)$

$$
\operatorname{dét}\left(\begin{array}{c}
\left\langle\boldsymbol{b}_{1}, \boldsymbol{h}_{1}^{+}\right\rangle \cdots\left\langle\boldsymbol{b}_{1}, \boldsymbol{h}_{l}^{-}\right\rangle \cdots\left\langle\boldsymbol{b}_{1}, \boldsymbol{h}_{m}^{+}\right\rangle \\
\vdots \\
\left\langle\boldsymbol{b}_{m}, \boldsymbol{h}_{1}^{+}\right\rangle \cdots\left\langle\boldsymbol{b}_{m}, \boldsymbol{h}_{l}^{-}\right\rangle \cdots\left\langle\boldsymbol{b}_{m}, \boldsymbol{h}_{m}^{+}\right\rangle
\end{array}\right)=0, \quad j=1, \cdots, m,
$$

où $\boldsymbol{h}_{j}^{ \pm}=\boldsymbol{h}_{j}^{ \pm}(\xi ; k+i 0), j=1, \cdots, m$. En tenant compte de l'expression (2.17) de $\tau_{1}^{ \pm}(\xi ; \lambda)$ et de la construction des $\boldsymbol{h}_{j}^{ \pm}(\xi ; \lambda)$ dans le $\S 2$ et en développant le dénominateur et le numérateur de

$$
\frac{\operatorname{dét}\left(\begin{array}{c}
\left\langle\boldsymbol{b}_{1}, \boldsymbol{h}_{1}^{+}\right\rangle \cdots\left\langle\boldsymbol{b}_{1}, \boldsymbol{h}_{l}^{-}\right\rangle \cdots\left\langle\boldsymbol{b}_{1}, \boldsymbol{h}_{m}^{+}\right\rangle \\
\vdots \\
\left\langle\boldsymbol{b}_{m}, \boldsymbol{h}_{1}^{+}\right\rangle \cdots\left\langle\boldsymbol{b}_{m}, \boldsymbol{h}_{l}^{-}\right\rangle \cdots\left\langle\boldsymbol{b}_{m}, \boldsymbol{h}_{+}^{m}\right\rangle
\end{array}\right)}{\left[\frac{d}{d \tau}\{(\tau I-M(\xi ; \lambda))\}\right]_{\tau=\tau_{1}-(\xi ; \lambda)}}
$$

par rapport à $\lambda$ au voisinage de $\beta(\xi)$ (voir (2.17)) respectivement, on voit que les fonctions (5.25) sont bornées uniformément pour $\lambda \in \bar{\Lambda}_{\delta}^{+}$. On en déduit l'intégrabilité uniforme du terme en question et l'assertion pour $\nu=0$ du théorème 5.1. Nous avons discuté, pour simplifier l'écriture, le cas oú $\nu=0$, mais on peut raisonner pour $\nu$ quelconque de la même manière qu'au cas où $\nu=0$.

En combinant ce théorème 5.1 avec le théorème 4.1, on a le

Théorème 5. 2. Pour tout $\nu$ et pour $x, y \in \boldsymbol{R}_{+}^{n}$ tels que $x \neq y$ les limites suivantes existent

$$
G^{(\nu)}(x, y ; k \pm i 0)=\lim _{\varepsilon \downarrow 0}\left(\frac{\partial}{\partial x}\right)^{\nu} G(x, y ; k \pm i \varepsilon)
$$


et $G^{(\nu)}(x, y ; k \pm i 0)$ sont continues par rapport $\dot{a}(x, y, k)$ dans $\left\{(x, y, k) ; x, y \in \mathbb{R}_{+}^{n}, x \neq y\right.$ et $\left.k \neq 0\right\}$.

Corollaire. Soit $g \in C_{0}^{\infty}\left(\boldsymbol{R}_{+}^{n}\right)$. Alors les limites suivantes

$$
G_{k \pm i_{0}}^{(\nu)} \cdot g(x)=\lim _{\varepsilon \downarrow 0}\left(\frac{\partial}{\partial x}\right)^{\nu} \int_{\mathbb{R}_{+}^{n}} G(x, y ; k \pm i \varepsilon) g(y) d y
$$

existent pour $x \in \boldsymbol{R}_{+}^{n}$ et $k \neq 0$ et de plus $G_{k \pm i 0}^{(\nu)} \cdot g(x)$ sont des fonctions continues de $(x, k)$ dans $\boldsymbol{R}_{+}^{n} \times\left(\boldsymbol{R}^{1}-\{0\}\right)$.

\section{§6. Démonstration du Théorème Principal}

La description de ce paragraphe est la même que celle du $\S 5$ de [7] à peu de notations près. Néanmoins pour compléter nous répétrons ici la discussion. Dans ce paragraphe nous revenons au problème original. Nous allons d'abord récrire le problème mixte (1.2)-(1.4) sous la forme d'une équation d'évolution dans $L^{2}\left(\boldsymbol{R}_{+}^{n}\right)$. L'opérateur différentiel $A=-i \sum_{j=1}^{n} A_{j} \frac{\partial}{\partial x_{j}}$ définit une transformation linéaire $\mathcal{A}$ non bornée dans $L^{2}\left(\boldsymbol{R}_{+}^{n}\right)$ de domaine de définition :

$$
D(\AA)=\left\{v(x) ; v \in C_{0}^{\infty}\left(\boldsymbol{R}_{+}^{n}\right), B v\left(x^{\prime}, 0\right)=0\right\} .
$$

L'opérateur $\mathcal{A}$ est fermable et nous désignons par $\boldsymbol{A}$ sa fermeture. On a alors le

Lemme 6.1. A est un opérateur autoadjoint (strict) dans l'espace hilbertien $L^{2}\left(\boldsymbol{R}_{+}^{n}\right)$ de domaine

$$
D(\boldsymbol{A})=\left\{v(x) ; v \in H^{1}\left(\boldsymbol{R}_{+}^{n}\right), B v(x)_{x_{n}=0}=0\right\},
$$

où l'on interprète $v(x)_{x_{n}=0}$ comme la trace de $v(x)$ sur l'hyperplan $x_{n}=0$.

Preuve. Ceci est dû à Lax et Phillips [4], [5]. Donc nous bornons ici à esquisser le procédé de la démonstration. L'adjoint formel $A^{(*)}$ de l'opérateur différentiel $A$ coïncide avec $A$ lui-même puisque les matrices $A_{j}, j=1, \cdots, n$, sont supposées hermitiennes. Soit $\mathscr{A}^{(*)}$ l'opérateur dans $L^{2}\left(\boldsymbol{R}_{+}^{n}\right)$ défini par $A^{(*)}=A$ de domaine

$$
D\left(\mathcal{A}^{(*)}\right)=\left\{v(x) ; v \in C_{0}^{\infty}\left(\boldsymbol{R}_{+}^{n}\right), v(x)_{x_{n}=0} \in\left(A_{n} \mathscr{B}\right)^{\perp}\right\} \cdot{ }^{14)}
$$

14) $\mathscr{R}$ un sous-ensemble de $\boldsymbol{C}^{2 m}$. $\mathscr{R} \perp$ désigne le sous-espace des vecteurs orthogonaux aux éléments de $\mathscr{R}$ par rapport au produit scalaire ordinaire. 
Alors la fermeture de $\mathcal{A}^{(*)}$ coïncide avec l'adjoint strict $\boldsymbol{A}^{*}$ de $\boldsymbol{A}$ au sens de la théorie de l'espace hilbertien ([4], p. 430). Pour démontrer $\boldsymbol{A}=\boldsymbol{A}^{*}$, il nous reste à montrer

$$
\mathscr{B}=\left(A_{n} \mathscr{B}\right)^{\perp} \quad \text { où } \quad \mathscr{B}=\operatorname{ker} B .
$$

Du fait que $B$ est conservatif, il découle $\mathscr{B} \subset\left(A_{n} \mathscr{B}\right)^{\perp}$. Or on a $\operatorname{dim} \mathscr{B}=m$ en vertu du lemme 1.1 sur la structure de $\mathscr{B}$. D'où, $\operatorname{dim}\left(A_{n} \mathscr{B}\right)^{\perp}=m$. Donc on a (6.4). D'autre part on a (6.2) de l'ellipticité de $A$ et de la coercivité de $B$.

En utilisant l'opérateur autoadjoint $A$ défini comme plus haut, on peut écrire le problème (1.2)-(1.4) sous la forme de l'équation d'évolution

$$
\begin{aligned}
& \frac{d}{d t} u(t)=i \boldsymbol{A} u(t), \\
& u(0)=g \in D(\boldsymbol{A}) .
\end{aligned}
$$

On considère comme solution de cette équation une fonction $t \mapsto u(t)$ $\in D(\boldsymbol{A})$, une fois continûment différentiable en $t$ dans $L^{2}\left(\boldsymbol{R}_{+}^{n}\right)$ (au sens de la topologie forte), vérifiant l'équation (6.5) et (6.6). On peut alors interpréter cette solution comme solution du problème mixte (1. 2)-(1.4).

L'opérateur autoadjoint $\boldsymbol{A}$ admet la décomposition spectrale

$$
\boldsymbol{A}=\int_{-\infty}^{+\infty} k d \mathbb{E}_{k}
$$

où $\left\{\boldsymbol{E}_{k}\right\}_{-\infty<k<\infty}$ est une famille spectrale déterminée par $\boldsymbol{A}$ d'une manière univoque. On peut supposer $\boldsymbol{E}_{k+0}=\boldsymbol{E}_{k}$ sans diminuer la généralité. On sait bien la solution unique du problème (6.5)-(6.6) est donnée par la formule

$$
u(t)=e^{i t A} g=\int_{-\infty}^{+\infty} e^{i t k} d \boldsymbol{E}_{k} g .
$$

Nous allons maintenant établir une relation entre la famille spectrale $\left\{\mathbb{E}_{k}\right\}_{-\infty<k<\infty}$ et le noyau de Green $G(x, y ; \lambda)$ du problème aux limites $\{A-\lambda I, B\}$, construit au paragraphe précédent.

Théorème 6.1. Soit $g \in C_{0}^{\infty}\left(\boldsymbol{R}_{+}^{n}\right)$. Alors

$1^{\circ} \quad \boldsymbol{E}_{k} g$ est une fonction continue de $(x, k)$ dans $\boldsymbol{R}_{+}^{n} \times \boldsymbol{R}^{1}$, 
$2^{\circ} \quad \boldsymbol{E}_{k} g$ est une fonction continûment différentiable en $k$ ( $x$ étant fixé) excepté $k=0$ et de plus on a

$$
\frac{d}{d k} \boldsymbol{E}_{k} g(x)=\frac{1}{2 \pi i}\left\{G_{k+i 0} \cdot g(x)-G_{k-i 0} \cdot g(x)\right\}
$$

Démonstration. Montrons d'abord que pour $k$ fixé, $\boldsymbol{E}_{k} g$ est une fonction indéfiniment dérivable en $x$ dans $\boldsymbol{R}_{+}^{n}$. L'élément $\boldsymbol{E}_{k} g$ peut être représenté comme une intégrale

$$
\boldsymbol{E}_{k} g=\int_{-\infty}^{k} d \boldsymbol{E}_{\sigma} g
$$

D’où il résulte

$$
\boldsymbol{A}^{p} \boldsymbol{E}_{k} g=\int_{-\infty}^{k} \sigma^{p} d \boldsymbol{E}_{\sigma} g
$$

Ceci implique $\boldsymbol{E}_{k} g \in D\left(\boldsymbol{A}^{p}\right)$ pour tout entier $p \geqslant 0$. Car on a évidemment $C_{0}^{\infty}\left(\boldsymbol{R}_{+}^{n}\right) \subset D\left(\boldsymbol{A}^{p}\right)$, d'où

$$
\left\|\boldsymbol{A}^{p} \boldsymbol{E}_{k} g\right\|^{2}=\int_{-\infty}^{k} \sigma^{2 p} d|| \boldsymbol{E}_{\sigma} g \|^{2}<+\infty
$$

Comme $\boldsymbol{A}$ est un opérateur linéaire dans $L^{2}\left(\boldsymbol{R}_{+}^{n}\right)$ associé au problème coercif aux limites pour l'opérateur elliptique $A$, on a en utilisant le lemme de Sobolev

$$
\bigcap_{p=1}^{\infty} D\left(\boldsymbol{A}^{p}\right) \subset \mathscr{B}\left(\overline{\boldsymbol{R}_{+}^{n}}\right)
$$

Ainsi on voit que pour $g \in C_{0}^{\infty}\left(\boldsymbol{R}_{+}^{n}\right), \boldsymbol{E}_{k} g$ peut être identifié avec un élément de l'espace $\mathscr{B}\left(\boldsymbol{R}_{+}^{\bar{n}}\right)$ des fonctions indéfiniment dérivables dans $\overline{\boldsymbol{R}_{+}^{n}}$, dont toutes les dérivées sont bornées. Pour montrer la continuité et la différentiabilité de $\boldsymbol{E}_{k} g$ par rapport à $k$, il faut utiliser une relation avec $G_{\lambda} \cdot g$. Pour $\lambda$ non réel, l'opérateur $(\boldsymbol{A}-\lambda \boldsymbol{I})^{-1}$ admet, comme fonction de $\boldsymbol{A}$, la représentation

$$
(\boldsymbol{A}-\lambda \boldsymbol{I})^{-1}=\int_{-\infty}^{+\infty} \frac{1}{k-\lambda} d \boldsymbol{E}_{k} .
$$

D'autre part nous avons la relation

$$
(\boldsymbol{A}-\lambda \boldsymbol{I})^{-1} g=\int_{\boldsymbol{R}_{+}^{n}} G(\cdot, y ; \lambda) g(y) d y .
$$


Par conséquent on a pour $\psi$ arbitraire dans $C_{0}^{\infty}\left(\boldsymbol{R}_{+}^{n}\right)$

$$
\int_{-\infty}^{+\infty} \frac{1}{k-\lambda} d\left(\boldsymbol{E}_{k} g, \psi\right)=\int_{\boldsymbol{R}_{+}^{n}}\left\{\int_{\boldsymbol{R}_{+}^{n}} G(x, y ; \lambda) g(y) d y\right\} \overline{\Psi(x)} d x
$$

En y appliquant la formule d'inversion de Stieltjes, ${ }^{15)}$ on obtient

$$
\begin{aligned}
& \left(\frac{\boldsymbol{E}_{k}+\boldsymbol{E}_{k-0}}{2} g, \psi\right)-\left(\frac{\boldsymbol{E}_{h}+\boldsymbol{E}_{h-0}}{2} g, \psi\right) \\
& \quad=\lim _{\varepsilon \downarrow 0} \frac{1}{2 \pi i} \int_{h}^{k} d \sigma \int_{\boldsymbol{R}_{-}^{n}}\left\{G_{\sigma+i \varepsilon} \cdot g(x)-G_{\sigma-i \varepsilon} \cdot g(x)\right\} \overline{\psi(x)} d x .
\end{aligned}
$$

Dans le second membre, les fonctions $G_{\sigma+i \varepsilon} \cdot g(x)$ et $G_{\sigma-i \varepsilon} \cdot g(x)$ sont continues par rapport à $(\sigma, \varepsilon, x)$ dans la région $\{(\sigma, \varepsilon, x) ; \sigma \neq 0$, $\left.0 \leqslant \varepsilon<\varepsilon^{0}, x \in \boldsymbol{R}_{+}^{n}\right\}$ et le support de $\psi$ est compact. Il est donc d'après un théorème de Lebesgue, permis d'effectuer le passage à la limite sous le signe d'intégration.

Il s'ensuit alors

$$
\begin{aligned}
& \left(\frac{\boldsymbol{E}_{k}+\boldsymbol{E}_{k-0}}{2} g, \psi\right)-\left(\frac{\boldsymbol{E}_{h}+\boldsymbol{E}_{h-0}}{2} g, \psi\right) \\
& \quad=\frac{1}{2 \pi i} \int_{h}^{k} d \sigma \int_{\boldsymbol{R}_{+}^{n}}\left\{G_{\sigma+i 0} \cdot g(x)-G_{\sigma-i 0} \cdot g(x)\right\} \cdot \overline{\psi(x)} d x .
\end{aligned}
$$

Le second membre de (6.16) est continu par rapport à $k$ et $h$, et $\psi$ est arbitraire dans $C_{0}^{\infty}\left(\boldsymbol{R}_{+}^{n}\right)$. Par suite on a pour presque tout les $x$

$$
\boldsymbol{E}_{k} g(x)-\boldsymbol{E}_{h} g(x)=\frac{1}{2 \pi i} \int_{h}^{k}\left\{G_{\sigma+i 0^{\circ}} g(x)-G_{\sigma-i 0} \cdot g(x)\right\} d \sigma
$$

Comme deux membres sont des fonctions continues de $x$, cette égalité est valable pour tout $x \in \boldsymbol{R}_{+}^{n}$. D'où il résulte, en dérivant

15) Soit $\phi(k)$ une fonction à variation bornée dans $[-\infty,+\infty]$. Considérons la fonction suivante de $\lambda$, définie pour $\lambda$ non réel par

$$
\Phi(\lambda)=\int_{-\infty}^{+\infty} \frac{1}{k-\lambda} d \phi(k),
$$

l'intégrale étant prise au sens de Lebesgue-Stieltjes. On a alors la formule d' inversion de Stieltjes :

$$
\frac{\phi(k+0)+\phi(k-0)}{2}-\frac{\phi(h+0)+\phi(h-0)}{2}=\lim _{\varepsilon \downarrow 0} \frac{1}{2 \pi i} \int_{h}^{k}\{\Phi(\sigma+i \varepsilon)-\Phi(\sigma-i \varepsilon)\} d \sigma .
$$


par rapport à $k$,

$$
\frac{d}{d k} \boldsymbol{E}_{k} g(x)=\frac{1}{2 \pi i}\left\{G_{k+i 0} \cdot g(x)-G_{k-i 0} \cdot g(x)\right\}
$$

Il nous reste à montrer que $\boldsymbol{E}_{k} g$ est continu en $k=0$. Si $\boldsymbol{E}_{k}-\boldsymbol{E}_{\boldsymbol{k}-0} \neq 0$ pour $k=0, k=0$ est un spectre ponctuel, c'est-à-dire une valeur propre de l'opérateur $\boldsymbol{A}$. Cela signifie qu'il existe dans $L^{2}\left(\boldsymbol{R}_{+}^{n}\right)$ une solution non identiquement nulle du problème aux limites

$$
A v(x)=0 \quad \text { pour } x \in \boldsymbol{R}_{+}^{n}, \quad B v(x)_{x_{n}=0}=0
$$

D'autre part on déduit de la coercivité de $B$ et des lemmes 1.2 et 3.1 que le problème (6.18) n'admet aucune solution non identiquement nulle qui soit à carré intégrable sur $\boldsymbol{R}_{+}^{n}$. On arrive ainsi à une contradiction. Donc l'application $k \mapsto \boldsymbol{E}_{k} g \in L^{2}\left(\boldsymbol{R}_{+}^{n}\right)$ doit être continue à $k=0$ pour tout $g \in L^{2}\left(\boldsymbol{R}_{+}^{n}\right)$. On verra aussi que $\boldsymbol{E}_{k} g(x)$ est une fonction continue de $(x, k)$ dans $\boldsymbol{R}_{+}^{n} \times \boldsymbol{R}^{1}$. c.q.f.d.

Passons maintenant à la

Démonstration du théorème principal. Comme nous l'avons remarqé dans ce qui précède, la solution du problème mixte (1.2)(1.4) avec $g \in C_{0}^{\infty}\left(\boldsymbol{R}_{+}^{n}\right)$ est donnée par la formule (6.8)

$$
u(t, \cdot)=\int_{-\infty}^{+\infty} e^{i t k} d \boldsymbol{E}_{k} g
$$

Décomposons cette intégrale comme suit;

$$
\begin{aligned}
& =\int_{N}^{\infty}+\int_{\delta}^{N}+\int_{-\delta}^{\delta}+\int_{-N}^{-\delta}+\int_{-\infty}^{-N} \\
& =u_{1}(t, x)+u_{2}(t, x)+u_{3}(t, x)+u_{4}(t, x)+u_{5}(t, x),
\end{aligned}
$$

$N$ et $\delta$ étant des nombres positifs qui vont être déterminés plus tard. Considérons d'abord la fonction

$$
u_{1}(t, x)=\int_{N}^{\infty} e^{i t k} d \boldsymbol{E}_{k} g
$$

Nous avons :

$$
\left\|\boldsymbol{A}^{p} u_{1}(t, \cdot)\right\|^{2}=\int_{N}^{\infty} k^{2 p} d\left\|\boldsymbol{E}_{k} g\right\|^{2}<+\infty
$$


pour tout entier $p \geqslant 0$, puisque $g \in C_{0}^{\infty}\left(\boldsymbol{R}_{+}^{n}\right) \subset \prod_{p=1}^{\infty} D\left(\boldsymbol{A}^{p}\right)$.

Du lemme de Sobolev et de l'inégalité coercive pour l'opérateur $A$, il résulte

$$
\begin{aligned}
\left|u_{1}(t, x)\right| & \leqslant \text { const }\left\|u_{1}(t, \cdot)\right\|_{H^{[n / 2]+1}\left(\boldsymbol{R}_{+}^{n}\right)} \\
& \leqslant \text { const } \sum_{p=0}^{[n / 2]+1}\left\|\boldsymbol{A}^{p} u_{1}(t, \cdot)\right\| .
\end{aligned}
$$

Soit $\varepsilon$ un nombre positif arbitraire. De (6.20), (6.21) et (6.22), on a, en choisissant $N>0$ suffisamment grand

$$
\left|u_{1}(t, x)\right| \leqslant \operatorname{const} \sum_{p=0}^{[n / 2]+1} \int_{N}^{\infty} k^{2 p} d\left\|\boldsymbol{E}_{k} g\right\|^{2}<\varepsilon
$$

pour tout $t \in \boldsymbol{R}^{1}$ et tout $x \in \boldsymbol{R}_{+}^{n}$. Par le même raisonnement, on a aussi

$$
\left|u_{5}(t, x)\right|<\varepsilon \text { pour tout } t \in \boldsymbol{R}^{1} \text { et tout } x \in \boldsymbol{R}_{+}^{n} \text {. }
$$

Passons ensuite à $u_{3}(t, x)=\int_{-\delta}^{\delta} e^{i t k} d \boldsymbol{E}_{k} g$. De la même manière qu'au précédent, on a

$$
\left|u_{3}(t, x)\right| \leqslant \operatorname{const} \sum_{p=0}^{[n / 2]+1} \int_{-\delta}^{\delta} k^{2 p} d\left\|\boldsymbol{E}_{k} g\right\|^{2}
$$

D'où

$$
\left|u_{3}(t, x)\right| \leqslant \operatorname{const}\left(1+\delta^{2}+\cdots+\delta^{2[n / 2]+2}\right)\left(\left\|\boldsymbol{E}_{\delta} g\right\|^{2}-\left\|\boldsymbol{E}_{-\delta} g\right\|^{2}\right) .
$$

Si on prend $\delta$ suffisamment petit, on a, en vertu de la continuité de $\boldsymbol{E}_{k} g$ à $k=0$,

$$
\left|u_{3}(t, x)\right|<\varepsilon \quad \text { pour tout } t \in \boldsymbol{R}^{1} \text { et tout } x \in \boldsymbol{R}^{n} .
$$

Envisageons $u_{2}(t, x)=\int_{\delta}^{N} e^{i t k} d \boldsymbol{E}_{k} g$. En utilisant le théorème 6. 1 , on peut l'écrire sous la forme:

$$
u_{2}(t, x)=\frac{1}{2 \pi i} \int_{\delta}^{N} e^{i t k}\left\{G_{k+i 0} \cdot g(x)-G_{k-i 0} \cdot g(x)\right\} d k
$$

Pour un point $x$ arbitrairement fixé de $\boldsymbol{R}_{+}^{n}$, les $G_{k \pm i 0} \cdot g(x)$ sont des fonctions continues en $k$ dans l'intervalle $[\delta, N]$. Donc, si on pose $\Theta(k, x)=G_{k+i 0} \cdot g(x)-G_{k-i 0} \cdot g(x)$ pour chaque $x \in \boldsymbol{R}_{+}^{n}$, l'intégrale 


$$
\int_{\delta}^{N} e^{i t k} \Theta(k, x) d k
$$

tend vers zéro lorsque $t \rightarrow \infty$ (aussi $t \rightarrow-\infty$ ). Soit $K$ un ensemble compact quelconque de $\boldsymbol{R}_{+}^{n}$. Alors la famille $\{\Theta(\cdot, x)\}_{x \in K}$ est précompacte dans l'espace des fonctions continues sur l'intervalle $[\delta, N]$. Car les fonctions $\Theta(k, x)$ et $\frac{\partial \Theta}{\partial x_{j}}(k, x)$ sont continues par rapport à $(k, x)$ dans $[\delta, N] \times K$, par conséquent, les fonctions $\{\Theta(\cdot, x)\}_{x \in K}$ de $k$ sont uniformément bornées. Par suite $\sup _{x \in K}\left|u_{2}(t, x)\right|$ tend vers zéro lorsque $t \rightarrow \infty$ (par exemple, d'après le théorème de BanachSteinhaus). Par le même raisonnement, $\sup _{x \in K}\left|u_{4}(t, x)\right|$ tend vers zéro lorsque $t \rightarrow \infty$. De (6.23), (6.24), (6.25) et l'énoncé ci-dessus, il suit que $u(t, x)$ converge vers zéro uniformément sur tout compact de $\boldsymbol{R}_{+}^{n}$, lorsque $t$ tend vers l'infini. On peut raisonner de la même manière sur ses dérivées.

c. q. f. d.

\section{§ 7. Appendice}

Nous donnons ici des exemples.

Exemple 1. Soit $L$ l'opérateur suivant.

$$
L=I \frac{\partial}{\partial t}-A_{1} \frac{\partial}{\partial x_{1}}-A_{2} \frac{\partial}{\partial x_{2}} \quad \text { où } A_{1}=\left(\begin{array}{ll}
0 & 1 \\
1 & 0
\end{array}\right) \text { et } A_{2}=\left(\begin{array}{rr}
1 & 0 \\
0 & -1
\end{array}\right) .
$$

Comme le polynôme caractéristique de $L$ est de la forme:

$$
\operatorname{dét}\left(\lambda I-\eta_{1} A_{1}-\eta_{2} A_{2}\right)=\lambda^{2}-\eta_{1}^{2}-\eta_{2}^{2},
$$

l'opérateur $L$ satisfait aux conditions L. 1)-L. 3) dans le $\S 1$. Nous allons considérer le problème mixte pour $L$ dans le demi-espace $\boldsymbol{R}_{+}^{2}=\left\{x=\left(x_{1}, x_{2}\right) ; x_{2}>0\right\}$ :

$$
\begin{array}{ll}
L[u(t, x)]=0 & \text { pour } t>0, x \in \boldsymbol{R}_{+}^{2}, \\
u(0, x)=g(x) & \text { pour } x \in \boldsymbol{R}_{+}^{2}, \\
\left.B u(t, x)\right|_{x_{n}=0}=0 & \text { pour } t>0 .
\end{array}
$$

La forme quadratique associée à $A_{2}$ est de la forme :

$$
A_{2} \zeta \cdot \bar{\zeta}=\left|\zeta_{1}\right|^{2}-\left|\zeta_{2}\right|^{2}, \quad \zeta \in C^{2} .
$$


Donc un espace-frontière $\mathscr{B}$ maximalement conservatif consiste en les multiples complexes d'un vecteur de la forme ${ }^{t}\left(1, e^{i \theta}\right), \theta$ étant un nombre réel tel que $0 \leqslant \theta<2 \pi$. Alors on peut supposer qu'une condition $\mathrm{B}$ aux limites minimalement conservative est de la forme:

$$
B=\left(-e^{i \theta}, 1\right) \quad(0 \leqslant \theta<2 \pi) .
$$

Désignons par $\tau^{+}=\tau^{+}(\xi ; \lambda)\left(\operatorname{resp} . \tau^{-}=\tau^{-}(\xi ; \lambda)\right.$ ) la racine à partie imaginaire positive (resp. négative) de l'équation en $\tau$ :

$$
\text { dét }(\tau I-M(\xi ; \lambda)) \equiv \operatorname{dét}\left(\tau I-A_{2}^{-1}(\lambda I-\xi A)\right) \equiv \tau^{2}-\lambda^{2}+\xi^{2}=0 .
$$

Nous remarquons ici le suivant:

$$
\tau^{+}(\xi ; k \pm i 0)= \begin{cases} \pm \operatorname{sgn} k \sqrt{k^{2}-\xi^{2}} & \text { pour }|\xi| \leqslant|k| \\ i \sqrt{\xi^{2}-k^{2}} & \text { pour }|\xi| \geqslant|k|,\end{cases}
$$

où $k$ est réel et $\sqrt{ } \bar{a}$ désigne la racine carrée positive d'un nombre $a>0$.

Compte tenu de $(7.5)$ et de ${ }^{t} \operatorname{cof}(\xi A+\tau A-\lambda I)=\left(\begin{array}{cc}-(\tau+\lambda) & -\xi \\ -\xi & \tau-\lambda\end{array}\right)$, on peut prendre, comme une base de $E^{+}(\xi ; \lambda)$, le vecteur ${ }^{t}\left(\tau^{+}(\xi ; \lambda)+\lambda, \xi\right)$ dans les cas suivants,

i) $\xi \neq 0$ et $\lambda$ non réel,

ii) $\xi \neq 0, \lambda=k$ réel et $|k|<|\xi|$,

iii) au voisinage de $\xi=0$ et $\operatorname{Im} \lambda>0$,

et le vecteur ${ }^{t}\left(-\xi, \tau^{\top}(\xi ; \lambda)-\lambda\right)$ au voisinage de $\xi=0$ pour $\operatorname{Im} \lambda<0$. D'où on peut prendre le déterminant de Lopatinski

(7. 6) $\Delta(\xi ; \lambda)=\operatorname{dét}\left\{\left(-e^{i \theta}, 1\right)\left(\begin{array}{c}-\left(\tau^{+}+\lambda\right) \\ -\xi\end{array}\right)\right\}=e^{i \theta}\left(\tau^{+}(\xi ; \lambda)+\lambda\right)-\xi$

pour les cas i) et iii), et

$$
=\operatorname{dét}\left\{\left(-e^{i \theta}, 1\right)\left(\begin{array}{c}
-\xi \\
\tau^{+}-\lambda
\end{array}\right)\right\}=e^{i \theta} \xi+\tau^{-1}(\xi ; \lambda)-\lambda
$$

au voisinage de $\xi=0$ pour $\operatorname{Im} \lambda<0$. D'où on déduit qu'il n'existe aucune condition $B=(\alpha, \beta)(\alpha, \beta \in \boldsymbol{C})$ aux limites vérifiant les conditions :

B. 1) B est minimalement conservative

B. 2$)^{*} \quad B$ satisfait à la condition complémentaire stricte $:^{16)}$

16) On appelle aussi cette condition "condition de Lopatinski uniforme". 
(7.7) $\Delta(\xi ; k \pm i 0) \neq 0$ pour $|\xi|+|k| \neq 0$. (Remarquons la condition $\Delta(\xi ; 0+i 0) \neq 0$ pour $|\xi| \neq 0$ equivaut à la coercivité de $B$ pour $L)$.

En effet on a $\Delta(\xi ; k \pm i 0)=0$ pour $k=\xi \cos \theta$. Mais il existe des conditions vérifiant B. 1) et B.2) dans le $\S 1$. En effet on a, d'après (7. 5$), E^{+}(\xi ; k)=\{0\}$ pour $|\xi| \leqslant|k|$ et $E^{+}(\xi ; k)=\left\{\alpha^{t}\left(i \sqrt{\xi^{2}-k^{2}}+k, \xi\right)\right.$; $\alpha \in \boldsymbol{C}\}$ pour $|\xi|>|k|$. Or

$$
(+1,1)\left(\begin{array}{c}
i \sqrt{\xi^{2}-k^{2}}+k \\
\xi
\end{array}\right) \neq 0 \quad \text { pour }|\xi|>|k| .
$$

Cela signifie que $B=\left(-e^{i \theta}, 1\right)$ satisfait à la condition B. 2$)$ pour $\theta=0, \pi$.

D'autre part il n'existe aucune condition $B=(\alpha, \beta)$ vérifiant B. 1) et B. 2) pour l'opérateur suivant dans $\boldsymbol{R}_{+}^{3}=\left\{x=\left(x_{1}, x_{2}, x_{3}\right) ; x_{3}>0\right\}$.

$$
\begin{aligned}
\text { (7.8) } L & =I \frac{\partial}{\partial t}-A_{1} \frac{\partial}{\partial x_{1}}-A_{2} \frac{\partial}{\partial x_{2}}-A_{3} \frac{\partial}{\partial x_{3}} \\
\text { où } & A_{1}=\left(\begin{array}{ll}
0 & 1 \\
1 & 0
\end{array}\right), \quad A_{2}=\left(\begin{array}{rr}
0 & i \\
-i & 0
\end{array}\right) \text { et } A_{3}=\left(\begin{array}{rr}
1 & 0 \\
0 & -1
\end{array}\right) .
\end{aligned}
$$

En effet on peut aisément constater qu'aucune condition aux limites, minimalement conservative n'est coercive pour $L$ (voir [5], p. 206).

Exemple 2. Soit $L$ l'opérateur suivant.

$$
\begin{aligned}
& L=I \frac{\partial}{\partial t}-A_{1} \frac{\partial}{\partial x_{1}}-A_{2} \frac{\partial}{\partial x_{2}} \\
& A_{1}=\left(\begin{array}{llll}
0 & 0 & 0 & 1 \\
0 & 0 & 2 & 0 \\
0 & 2 & 0 & 0 \\
1 & 0 & 0 & 0
\end{array}\right) \text { et } A_{2}=\left(\begin{array}{rrrr}
1 & 0 & 0 & 0 \\
0 & 2 & 0 & 0 \\
0 & 0 & -2 & 0 \\
0 & 0 & 0 & -1
\end{array}\right) .
\end{aligned}
$$

Le polynôme caractéristique de $L$ est donné par

$$
\text { dét }\left(\lambda I-\eta_{1} A_{1}-\eta_{2} A_{2}\right)=\left(\lambda^{2}-\eta_{1}^{2}-\eta_{2}^{2}\right)\left(\lambda^{2}-4 \eta_{1}^{2}-4 \eta_{2}^{2}\right) \text {. }
$$

Donc l'opérateur $L$ satisfait aux conditions L. 1)-L. 3). Considérons le problème mixte (1.2)-(1.4) pour $L$ dans $\boldsymbol{R}_{+}^{2}$. La forme quadratique associée $A_{2}$ est de la forme:

$$
A_{2} \zeta \cdot \bar{\zeta}=\left|\zeta_{1}\right|^{2}+2\left|\zeta_{2}\right|^{2}-2\left|\zeta_{3}\right|^{2}-\left|\zeta_{4}\right|^{2}, \quad \zeta \in \boldsymbol{C}^{4} .
$$


Considérons la condition aux limites:

$$
B=\left(\begin{array}{cccc}
1 & 0 & -\sqrt{2} & 0 \\
0 & -\sqrt{2} & 0 & 1
\end{array}\right)
$$

Alors l'espace-frontière $\mathscr{B}=\operatorname{ker} B$ consiste en les vecteurs

$$
\alpha^{t}(\sqrt{2}, 0,1,0)+\beta^{t}(0,1,0, \sqrt{2}), \alpha, \beta \in C .
$$

Par suite $B$ est minimalement conservative.

Compte tenu de $\operatorname{dét}(\tau I-M(\xi ; \lambda))=\left(\tau^{2}-\lambda^{2}+\xi^{2}\right)\left(\tau^{2}-\frac{\lambda^{2}}{4}+\xi^{2}\right)$, désignons par $\tau_{1}^{+}=\tau_{1}^{+}(\xi ; \lambda)$ la racine à partie imaginaire positive de $\tau^{2}=\lambda^{2}-\xi^{2}$ et par $\tau_{2}^{+}=\tau_{2}^{+}(\xi ; \lambda)$ celle de $\tau^{2}=\lambda^{2} / 4-\xi^{2}$ pour $\lambda$ non réel. Alors $\tau_{1}^{+}(\xi ; k \pm i 0)$ est de la forme (7. 5) et $\tau_{2}^{+}(\xi ; k \pm i 0)$ est de la forme suivante

$$
\tau_{2}^{+}(\xi ; k \pm i 0)= \begin{cases} \pm \operatorname{sgn} k \sqrt{k^{2} / 4-\xi^{2}} & \text { pour }|\xi| \leqslant|k| / 2 \\ i \sqrt{\xi^{2}-k^{2} / 4} & \text { pour }|\xi| \geqslant|k| / 2\end{cases}
$$

où $k$ est réel et $\sqrt{ } \bar{a}$ désigne la racine carrée positive de $a>0$. ${ }^{t} \operatorname{cof}\left(\xi A_{1}+\tau A_{2}-\lambda I\right)$ est donné par

$$
\left(\begin{array}{ccc}
(\tau+\lambda)\left(4 \xi^{2}+4 \tau^{2}-\lambda^{2}\right) & 0 & 0 \\
0 & (2 \tau+\lambda)\left(\xi^{2}+\tau^{2}-\lambda^{2}\right) & 2 \xi\left(\xi^{2}+\tau^{2}-\lambda^{2}\right) \\
0 & 2 \xi\left(\xi^{2}+\tau^{2}-\lambda^{2}\right) & (\lambda-2 \tau)\left(\xi^{2}+\tau^{2}-\lambda^{2}\right) \\
\xi\left(4 \xi^{2}+4 \tau^{2}-\lambda^{2}\right) & 0 & 0 \\
& \xi\left(4 \xi^{2}+4 \tau^{2}-\lambda^{2}\right) \\
& 0 \\
& 0 \\
& (\lambda-\tau)\left(4 \xi^{2}+4 \tau^{2}-\lambda^{2}\right)
\end{array}\right) .
$$

On peut choisir comme une base $\left\{\boldsymbol{h}_{j}^{+}(\xi ; \lambda)\right\}_{j=1,2}$ de $E^{+}(\xi ; \lambda)$ le suivant.

$$
\begin{aligned}
& \boldsymbol{h}_{1}^{+}(\xi ; \lambda)={ }^{t}\left(\tau_{1}^{+}(\xi ; \lambda)+\lambda, 0,0, \xi\right) \text { et } \\
& \boldsymbol{h}_{2}^{+}(\xi ; \lambda)={ }^{t}\left(0,2 \tau_{2}^{+}(\xi ; \lambda)+\lambda, 2 \xi, 0\right)
\end{aligned}
$$

pour $\xi \neq 0$ et $\lambda$ non réel ou au voisinage de $\xi=0$ et pour $\operatorname{Im} \lambda>0$. Pour Im $\lambda<0$ on doit prendre comme $\boldsymbol{h}_{1}^{+}$et $\boldsymbol{h}_{2}^{+}$au voisinage de $\xi=0$ les suivants 


$$
\begin{aligned}
& \boldsymbol{h}_{1}^{+}(\xi ; \lambda)={ }^{t}\left(\xi, 0,0, \lambda-\tau_{1}^{+}(\xi ; \lambda)\right) \text { et } \\
& \boldsymbol{h}_{2}^{+}(\xi ; \lambda)={ }^{t}\left(0,2 \xi, \lambda-2 \tau_{2}^{+}(\xi ; \lambda), 0\right)
\end{aligned}
$$

puisqu'on a $\boldsymbol{h}_{1}^{+}(0 ; k-i 0)=0$ et $\boldsymbol{h}_{2}^{+}(0 ; k-i 0)=0$ pour $\boldsymbol{h}_{j}^{+}(\xi ; \lambda) j=1,2$ donnés par (7.14).

Alors le déterminant de Lopatinski $\Delta(\xi ; \lambda)$ est donné par

$$
\Delta(\xi ; \lambda)=-\sqrt{2}\left\{\left(\tau_{1}^{+}(\xi ; \lambda)+\lambda\right)\left(2 \tau_{2}^{+}(\xi ; \lambda)+\lambda\right)-2 \xi^{2}\right\}
$$

pour $\xi \neq 0$ et $\lambda$ non réel, ou au voisinage de $\xi=0$ et pour $\operatorname{Im} \lambda>0$. On peut constater que $\Delta(\xi ; k+i 0) \neq 0$ pour $\xi \neq 0$ et $k$ réel. D'autre part, au voisinage de $\xi=0$ et pour $\operatorname{Im} \lambda<0, \Delta(\xi ; \lambda)$ est de la forme

$$
\Delta(\xi ; \lambda)=-\sqrt{2}\left\{2 \xi^{2}-\left(\lambda-\tau_{1}^{+}(\xi ; \lambda)\right)\left(\lambda-2 \tau_{2}^{+}(\xi ; \lambda)\right)\right\} .
$$

D’où $\Delta(0, k-i 0)=4 \sqrt{2} k^{2} \neq 0$ pour $k$ réel $\neq 0$. Ainsi $B=\left(\begin{array}{rrrr}1 & 0 & -2 & 0 \\ 0 & -2 & 0 & 1\end{array}\right)$ vérifie B. 1) et B. 2)*.

(Ce sont les hypothèses faites dans notre travail antérieur [7].) Il en est de même de l'opérateur suivant dans $\boldsymbol{R}_{+}^{3}$.

$$
L=I \frac{\partial}{\partial t}-A_{1} \frac{\partial}{\partial x_{1}}-A_{2} \frac{\partial}{\partial x_{2}}-A_{3} \frac{\partial}{\partial x_{3}},
$$

où $A_{1}=\left(\begin{array}{llll}0 & 0 & 0 & 1 \\ 0 & 0 & 2 & 0 \\ 0 & 2 & 0 & 0 \\ 1 & 0 & 0 & 0\end{array}\right), A_{2}=\left(\begin{array}{cccc}0 & 0 & 0 & i \\ 0 & 0 & -2 i & 0 \\ 0 & 2 i & 0 & 0 \\ -i & 0 & 0 & 0\end{array}\right)$ et $A_{3}=\left(\begin{array}{rrrr}1 & 0 & 0 & 0 \\ 0 & 2 & 0 & 0 \\ 0 & 0 & -2 & 0 \\ 0 & 0 & 0 & -1\end{array}\right)$.

Condition aux limites

$$
B=\left(\begin{array}{cccc}
1 & 0 & -\sqrt{2} & 0 \\
0 & -\sqrt{2} & 0 & 1
\end{array}\right) .
$$

Base $\left\{\boldsymbol{h}_{j}^{+}(\xi ; \lambda)\right\}_{j=1,2}$ de $E^{+}(\xi ; \lambda)$.

ou

$$
\left\{\begin{array}{l}
\boldsymbol{h}_{1}^{+}(\xi ; \lambda)={ }^{t}\left(\tau_{1}^{+}(\xi ; \lambda)+\lambda, 0,0, \xi_{1}-i \xi_{2}\right) \\
\boldsymbol{h}_{2}^{+}(\xi ; \lambda)={ }^{t}\left(0,2 \tau_{2}^{+}(\xi ; \lambda)+\lambda, 2\left(\xi_{1}+i \xi_{2}\right), 0\right)
\end{array}\right.
$$

$$
\left\{\begin{array}{l}
\boldsymbol{h}_{1}^{+}(\xi ; \lambda)={ }^{t}\left(\xi_{1}+i \xi_{2}, 0,0, \lambda-\tau_{1}^{+}(\xi ; \lambda)\right) \\
\boldsymbol{h}_{2}^{+}(\xi ; \lambda)={ }^{t}\left(0,2\left(\xi_{1}-i \xi_{2}\right), \lambda-2 \tau_{2}^{+}(\xi ; \lambda), 0\right) .
\end{array}\right.
$$


Déterminant de Lopatinski.

$$
\begin{aligned}
\Delta(\xi ; \lambda)=-\sqrt{2}\left\{\left(\tau_{1}^{+}+\lambda\right)\left(2 \tau_{2}^{+}+\lambda\right)-2\left(\xi_{1}^{2}+\xi_{2}^{2}\right)\right\} \\
\text { ou }-\sqrt{2}\left\{2\left(\xi_{1}^{2}+\xi_{2}^{2}\right)-\left(\lambda-\tau_{1}^{+}\right)\left(\lambda-2 \tau_{2}^{+}\right)\right\} .
\end{aligned}
$$

\section{Bibliographie}

[1] Agmon, S., Problèmes mixtes pour les équations hyperboliques d'ordre supérieur, Colloques sur les Équations aux Dérivées Partielles, C.N.R.S. (1962), 13-18.

[2] Carleman, T., Sur les Équations Intégrales Singulières à Noyau Réel et Symétrique, Uppsala, 1923.

[3] Hersh, R., Mixed problems in several variables, J. Math. Mech. 12 (1963), 317-334.

[4] Lax, P. D. and R. S. Phillips, Local boundary conditions for dissipative symmetric linear differential operators, Comm. Pure Appl. Math. 13 (1960), 427-455.

[5] — Scattering Theory, Academic Press, New York, 1967.

[6] Lax, P. D., C.S. Morawetz and R. S. Phillips, The exponential decay of solutions of the wave equation in the exterior of a star-shaped obstacle, Bull. Amer. Math. Soc. 68 (1962), 593-595 et Comm. Pure Appl. Math. 16 (1963), 477-486.

[7] Matsumura, M., Comportement des solutions de quelques problèmes mixtes pour certains systèmes hyperboliques symétriques à coefficients constants, Publ. RIMS Kyoto Univ. Ser. A, 4 (1968), 309-359.

[8] Comportement asymptotique de solutions de certains problèmes mixtes pour des systèmes hyperboliques symétriques à coefficients constants, Proceedings of the International Conference on Functional Analysis and Related Topics, Math. Soc. Japan, 1969.

[9] Mizohata, S., Sur l'analyticité de la fonction spectrale de l'opérateur relatif au problème extérieur, Proc. Japan Acad. 39 (1963), 352-357.

[10] - Problèmes mixtes pour les équations hyperboliques à coefficients constants, 1968 (en japonais).

[11] Morawetz, C. S., The decay of solutions of the exterior initial-boundary value problem for the wave equation, Comm. Pure Appl. Math. 14 (1961), 561-569.

[12] Mochizuki, K., The limiting amplitude principle for hyperbolic systems in an exterior domain, to appear.

[13] Sarason, L. On hyperbolic mixed problems, Arch. Rat. Mech. Anal. 18 (1965), 310-334.

[14] Strang, G., Hyperbolic initial-boundary value problems in two unknowns, to appear. 